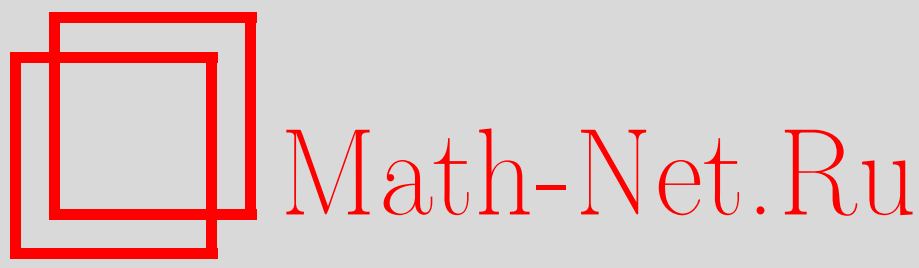

Б. В. Пальцев, О смешанной задаче с неоднородными граничными условиями для эллиптических с параметром уравнений второго порядка в липшицевых областях, Матем. сб., 1996, том 187, номер 4, 59-116

DOI: https://doi.org/10.4213/sm123

Использование Общероссийского математического портала Math-Net.Ru подразумевает, что вы прочитали и согласны с пользовательским соглашением

http: //www.mathnet.ru/rus/agreement

Параметры загрузки:

IP: 44.207 .124 .84

26 апреля 2023 г., 11:29:01 
УДК 517.956

\author{
Б.В. Пальцев
}

\title{
О смешанной задаче с неоднородными граничными условиями для эллиптических с параметром уравнений второго порядка в липшицевых областях
}

Для эллиптического с параметром уравнения второго порядка с дивергентной главной частью в липшицевой области $\Omega$ в рамках обобщенных решений из $W_{2}^{1}(\Omega)$ рассмотрены смешанные задачи (типа Зарембы) с неоднородными граничньми условиями. Изучаются операторы Пуанкаре-Стеклова на липшицевом куске $\gamma$ границы $\Gamma$, отвечающие однородным смешанным граничным условиям на $\Gamma \backslash \gamma$. В цилиндрической области с липшицевьпм сечением для однородного уравнения при однородных смешанных условиях на боковой поверхности и неоднородных граничных условиях на торцах при наличии разделения переменных проведено обоснование метода Фурье.

Библиографйя: 33 названия.

\section{$\S 1$. Введение}

Настоящая статья посвящена некоторым аспектам теории смешанной краевой задачи

$$
\begin{aligned}
& L(x, D, \mu) u(x)=f(x) \quad \text { в } \Omega, \\
& \left.u(x)\right|_{\Gamma_{1}}=\varphi(x),\left.\quad\left(\frac{\partial u}{\partial \nu}(x)+\sigma(x) u(x)\right)\right|_{\Gamma_{2}}=g(x)
\end{aligned}
$$

в липшицевой области $\Omega n$-мерного евклидова пространства $\mathbb{R}^{n}$, где $L(x, D, \mu)$ эллиптический с параметром $\mu$ с дивергентной главной частью оператор второго порядка

$$
\begin{aligned}
L(x, D, \mu) u(x)=-\sum_{i, j=1}^{n} D_{i}( & \left.a_{i j}(x) D_{j} u(x)\right) \\
& +\sum_{i=1}^{n} a_{i}(x) D_{i} u(x)+\left(a_{0}(x)+\mu^{2} q(x)\right) u(x)
\end{aligned}
$$

$\left(D_{j}=\partial / \partial x_{j}\right), \Gamma_{1}$ и $\Gamma_{2}-$ непересекающиеся части, составляющие границу $\Gamma$ области $\Omega$,

$$
\left.\frac{\partial u}{\partial \nu}(x)\right|_{\Gamma}=\left.\sum_{j=1}^{n}\left(\sum_{i=1}^{n} a_{i j}(x) n_{i}(x)\right) D_{j} u(x)\right|_{\Gamma}
$$

Работа выполнена при финансовой поддержке Российского фонда фундаментальных исследований (грант № 93-01-00606). 
- значения на Г конормальной производной от $u\left(n_{i}(x)\right.$ - компоненты единичной внешней нормали $\boldsymbol{n})$. Предполагается, что коэффициенты $a_{i j}(x), a_{i}(x), a_{0}(x)$ в обшем случае комплексные, $q(x)$ - действительная функция из $L_{\infty}(\Omega)$, матрица $a_{i j}(x)$ эрмитова и равномерно положительная на $\Omega: a_{i j}(x)=\overline{a_{j i}(x)}$,

$$
\sum_{i, j=1}^{n} a_{i j}(x) \zeta_{i} \bar{\zeta}_{j} \geqslant \alpha_{0}|\zeta|^{2}, \quad \alpha_{0}>0, \quad \forall \zeta \in \mathbb{C}^{n}, \quad \forall x \in \Omega
$$

$q(x)$ - также равномерно положительна на $\Omega$ :

$$
q(x) \geqslant q_{0}>0 \quad \forall x \in \Omega
$$

где $\alpha_{0}, q_{0}$ - постоянные. Коэффициент $\sigma(x)$ также предполагается в общем случае ограниченной измеримой функцией на $\Gamma_{2}$. Параметр $\mu$ допускается комплексным, и может быть по модулю большим.

Теория общих краевых задач для эллиптических уравнений порядка $2 m, m-$ натуральное, с граничными условиями, удовлетворяющими в каждой точке условиям совместности (условиям Шапиро-Лопатинского), представляет собой вполне сложившуюся и хорошо разработанную теорию. Обычно здесь рассмотрения ведутся для областей с достаточно гладкими или вообще бесконечно дифференцируемыми границами и для уравнений с достаточно гладкими коэффициентами, см., например, [1]-[6]. Имеются направления, посвященные изучению эллиптических задач в областях с негладкими границами; из работ этих направлений отметим здесь лишш [7]-[9], см. также имеющиеся там ссылки.

Что касается общей теории смешанных эллиптических краевых задач, т.е. задач, для которых на двух или нескольких участках границы задаются граничные условия различных типов, то построение такой теории оказывается гораздо сложнее, и ее пока вряд ли можно считать полностью завершенной. По этой тематике отметим только работы [10]-[14], см. также некоторые главы в [9], (см. еще некоторые ссылки в [14]). В [10] был получен первый результат по существованию классического решения смешанной задачи для уравнения Лапласа и оценке первых производных вблизи поверхности $\bar{\Gamma}_{1} \cap \bar{\Gamma}_{2}$. В [11]-[14] изучались обшие смешанные граничные задачи для эллиптических уравнений порядка $2 m$ (в [13] с $m=1$ ), а в [14] даже и для систем, эллиптических по Дуглису-Ниренбергу. В [13] и [14], а также в других работах этих авторов на основе существенного развития методики Винера-Хопфа была построена общая теория эллиптических краевых задач для псевдодифференциальных уравнений в ограниченной области, и, как следствие этой теории, общая теория смешанных эллиптических граничных задач. При этом для обеспечения нормальной разрешимости в пространствах типа Соболева-Слободецкого задачи в общем случае рассматривались в обобшенной постановке: в граничные условия вводились операторы типа потенциала с неизвестными плотностями и на $\bar{\Gamma}_{1} \cap \bar{\Gamma}_{2}$ задавались дополнительные граничные условия; изучалось асимптотическое поведение решений в окрестности $\bar{\Gamma}_{1} \cap \bar{\Gamma}_{2}$. Отметим однако, что во всех этих работах (за исключением [9], где смешанные задачи рассмотрены в плоских полигональных областях) рассмотрения проводились в областях 
с достаточно гладкими границами (заведомо более гладкими, чем общие липшицевы) и для решений с более высокой гладкостью (для уравнения 2-го порядка в этих работах решение рассматривается, как минимум, из пространств $W_{2}^{l}(\Omega)$, где $l>3 / 2)$.

Данная работа возникла из потребностей и в процессе обоснования итерационных методов с разделением на подобласти без перекрытия численного решения краевых задач для эллиптических уравнений второго порядка. Такие методы имеют очень высокую скорость сходимости для диссипативных уравнений с большим действительным параметром $\mu$ в (1.1), (1.3). На практике обычно при разделении расчетной области (особенно, если подстраиваться под используемые сетки) возникают подобласти, границы которых могут иметь углы, ребра, пирамидальные вершины и т. д. Построение и исследование подобных методов базируются на исследовании возникающих на разделяющих поверхностях операторов типа Пуанкаре-Стеклова и обратных к ним, см., например, работы [15], [16], в которых теория операторов Пуанкаре-Стеклова получила существенное развитие. При этом требуется точное описание функциональных пространств, в которых действуют такие операторы, а также соответствующие оценки для них.

Для возникающих (в подобластях) смешанных задач вида (1.1), (1.2), которые являются вариационньми (см. [7], [9], [17]-[20]), оказывается как раз весьма удобным расширение класса рассматриваемых областей до липшицевых. Для таких задач в таких областях сама теория разрешимости в пространстве Соболева $W_{2}^{1}(\Omega)$ (см. [20]) так же проста, как и для задач Дирихле или Неймана в областях с гладкими границами. В то же время имеющиеся тут простые и тем не менее точные результаты оказываются достаточными и хорошо приспособленньми для ряда приложений, например, для упомянутых вьше.

Содержание статьи следуюшее. В $§ 2$ приводятся необходимые сведения из теории применяемых функциональных пространств. Для задач с параметром вида $(1.1),(1.2)$, как и в [4], используется нормировка соболевских пространств со специальным вхождением параметра $\mu$. В $\S 3$ приводится доказательство теоремы о фредгольмовости задачи (1.1), (1.2) и сопряженной к ней и об однозначности разрешимости этих задач при достаточно больших Re $\mu$. При этом перед формулировкой теоремы обсуждается понятие конормальной производной на Г от обобщенных решений задач. Четвертый параграф посвящен теореме о разложениях по обобшенным собственным функциям эллиптического оператора (1.3) с однородными граничными условиями (1.2) в самосопряженном случае. Эта теорема является непосредственньм обобщением результатов, представленных, например, в [18] для случая задачи Дирихле или 3-ей краевой в более гладких областях. В $§ 5$ рассматриваются операторы Пуанкаре-Стеклова на открытом куске $\gamma$ границы Г для оператора (1.3) в том случае, когда на остальной части $\Gamma \backslash \gamma$, разбитой на два куска $\Gamma_{1}$ и $\Gamma_{2}$, задаются однородные граничные условия вида (1.2). В самосопряженном случае описываются спектральные свойства такого оператора. Отметим, что в [15] и [16] операторы Пуанкаре-Стеклова изучались для вариационных задач в абстрактной форме, но, по-сушеству, только для случая, когда $\Gamma_{2}=\varnothing$. В последнем $\S 6$ рассматриваются смешанные задачи для однородного уравнения в цилиндрической области с липшицевым сечением $\Lambda$ с однородными смешанными условиями на боковой поверхности цилиндра и с неоднородньпи условиями Дирихле или 
Неймана на торцах. Задачи в конечном итоге формулируются как задачи с заданием данных на торцах как функций или функционалов на сечении $\Lambda$ из вводимых пространств $\stackrel{\circ}{W} \underset{2, \mu}{1 / 2}\left(\Lambda ; \gamma_{1}\right)$ или $\left.\stackrel{\circ}{W}-1 / 2, \mu ; \gamma_{1}\right)$, описываемых внутренним образом. При этом $\gamma_{1}$ предполагается липшицевым куском $\partial \Lambda$. При использовании результатов $\S 5$ получено обоснование метода Фурье для такого вида смешанных задач, допускающих разделение переменных. Установлена сходимость соответствующих рядов в $W_{2}^{1}(\Omega)$; для самосопряженного оператора (1.3) со смешанными однородными условиями вида (1.2), в частности, установлена базисность собственных функций в пространствах $\stackrel{\circ}{W} \underset{2, \mu}{1 / 2}\left(\Omega ; \Gamma_{1}\right)$ и $\stackrel{\circ}{W_{2, \mu}^{-1 / 2}}\left(\Omega ; \Gamma_{1}\right)$.

Автор выражает благодарность В.П. Михайлову, В.И. Агошкову, А. К. Гущину и А.А. Дезину за ряд плодотворных обсуждений и полезных замечаний.

\section{§2. Пространства Соболева-Слободецкого в липшицевых областях}

В этом параграфе сформулируем необходимые для дальнейшего определения и результаты, сопровождая их в основном лишь комментариями и пояснениями. По поводу материала данного параграффа см. [20]-[28], [4], [5], [7] и [9]. Обобщение теории функциональных пространств Соболева $W_{2}^{l}(\Omega)$ на случай нецелых $l$ в областях, а также на многообразиях более гладких, чем липшицевы, было построено в [22]. В [21] было получено описание следов функций из пространства $W_{p}^{1}(\Omega), p>1$, на липшицеву границу. Перенесение теории пространств Соболева-Слободецкого и развитой в [22] техники локализации с применением преобразования Фурье на случай липшищевых областей позволяют осуществить важные результаты о дифференцируемости почти всюду (п.в.) непрерывных по Липшицу функций и о справедливости классической формулы замены переменных в кратных интегралах при билипшицевых отображениях. Эти результаты, причем даже для более общего класса функций, были впервые получены в [27]; отметим также работу [28], в которой теорема о дифференцируемости п.в. липшиц-непрерывных функций, дополнительно с некоторыми важньми подробностями, установлена проще.

Точки в $\mathbb{R}^{n}$ будем обозначать, как и выше, латинскими маленькими буквами $x$, $y$ и т. д., а иногда и большими буквами $P, M$ и т. д.

ОПРЕДЕЛЕНИЕ 2.1 (см. [7], [9], [19], [21], [23], [24], [26]). Ограниченная область $\Omega \subset \mathbb{R}^{n}$ называется липшицевой областью или областью с липшицевой границей $\Gamma$, если для любой точки (т.) $P \in \Gamma$ найдется такая декартова система координат $\xi$ с началом в т. $P, \xi=\left(\xi^{\prime}, \xi_{n}\right), \xi^{\prime}=\left(\xi_{1}, \ldots, \xi_{n-1}\right)$ и такая окрестность $O(P)=\left\{x \in \mathbb{R}^{n}:\left|\xi^{\prime}\right|<r_{P},\left|\xi_{n}\right|<\delta_{P}\right\}, r_{P}>0, \delta_{P}>0$, что часть $\Gamma \cap O(P)$ границы Г имеет в этой системе $\xi$ представление

$$
\xi_{n}=F_{P}\left(\xi^{\prime}\right) \forall \xi^{\prime} \in O^{\prime}(P)=\left\{\xi^{\prime}:\left|\xi^{\prime}\right|<r_{P}\right\}
$$

с функцией $F_{P}\left(\xi^{\prime}\right)$ непрерывной по Липшицу:

$$
\left|F_{P}\left(\xi^{\prime}\right)-F_{P}\left(\eta^{\prime}\right)\right| \leqslant L_{P}\left|\xi^{\prime}-\eta^{\prime}\right| \forall \xi^{\prime}, \eta^{\prime} \in O^{\prime}(P),
$$

где $L_{P}$ - некоторая постоянная для $O^{\prime}(P)$, причем найдется такое $h_{P}>0$, что множество

$$
\left\{x \in \mathbb{R}^{n}: F_{P}\left(\xi^{\prime}\right)-h_{P}<\xi_{n}<F_{P}\left(\xi^{\prime}\right), \xi^{\prime} \in O^{\prime}(P)\right\}
$$


принадлежит $\Omega$, а множество

$$
\left\{x \in \mathbb{R}^{n}: F_{P}\left(\xi^{\prime}\right) \leqslant \xi_{n}<F_{P}\left(\xi^{\prime}\right)+h_{P}, \xi^{\prime} \in O^{\prime}(P)\right\}
$$

не имеет общих точек с $\Omega$. Такие декартову систему $\xi$ и окрестность $O(P)$ будем называть подходящими в т. $P \in \Gamma$.

Естественно, $O^{\prime}(P)$ можно брать не шаровой, а какой-либо другой, например, прямоугольной.

Для любой т. $\xi^{\prime} \in O^{\prime}(P)$ будем обозначать через $M_{\xi^{\prime}}$ точку из $\Gamma \cap O(P)$ с координатами $\left(\xi^{\prime}, F_{P}\left(\xi^{\prime}\right)\right)$ в подходящей системе $\xi$, относяшейся к т. $P$. Окрестность $O^{\prime}(P)$ с отображением $\xi^{\prime} \leftrightarrow M_{\xi^{\prime}}$ назовем подходящей локальной картой Г в окрестности т. $P \in \Gamma$; набор $\xi^{\prime}$ представляет собой локальные координаты т. $M_{\xi^{\prime}} \in \Gamma$ в этой карте.

Если $f(M), M \in \Gamma,-$ функция, определенная на $\Gamma$, то для всякой $P \in \Gamma$ и для всяких подходящих декартовой системы $\xi$, окрестности $O(P)$ и локальной карты $O^{\prime}(P)$ функция

$$
\widehat{f}_{P}\left(\xi^{\prime}\right)=f\left(M_{\xi^{\prime}}\right) \forall \xi^{\prime} \in O^{\prime}(P)
$$

представляет собой выражение функции $f(M)$ в локальной карте $O^{\prime}(P)$. Если каждая из функций $\widehat{f}_{P}\left(\xi^{\prime}\right)$ для каждой т. $P \in \Gamma$ и какого-нибудь одного набора (для т. $P$ ) подходяших системы $\xi$ и окрестности $O(P)$ измерима по Лебегу на карте $O^{\prime}(P)$, то $f(M)$ называется измеримой на $Г$.

В силу теоремы Радемахера [27] (см. также [28]) и свойства $(2.2)$ функция $F_{P}\left(\xi^{\prime}\right)$ в представлении (2.1) п.в. дифференцируема на $O^{\prime}(P)$, а потому при п.в. $\xi^{\prime} \in O^{\prime}(P)$ в точках $M_{\xi^{\prime}} \in \Gamma$ сушествует касательная плоскость к $\Gamma$, нормальный вектор к которой

$$
\boldsymbol{n}=\left[1+\sum_{k=1}^{n-1}\left(\frac{\partial F_{P}}{\partial \xi_{k}}\right)^{2}\right]^{-1 / 2}\left\{-\frac{\partial F_{P}}{\partial \xi_{1}}, \ldots,-\frac{\partial F_{P}}{\partial \xi_{n-1}}, 1\right\}
$$

следует понимать как единичньй вектор внешней нормали к Г в т. $M_{\xi^{\prime}}$. В силу $(2.2)$ $\left|\frac{\partial F_{P}}{\partial \xi_{k}}\right| \leqslant L_{P}$.

Интеграл $\int_{\Gamma} f(M) d s$ по липшицевой границе Г от измеримой функции $f(M)$ определяется с помощью локализации с применением гладкого разбиения единицы, подчиненного некоторому покрытию Г подходящими окрестностями, и вычисления соответствующих интегралов в локальных картах точно так же, как и по гладкой границе. Если $\Lambda$ - некоторое подмножество $Г$, то $\Lambda$ называется измеримымм на $\Gamma$, если измерима характеристическая функция $\chi_{\Lambda}(x)$ множества $\Lambda$. Ин-

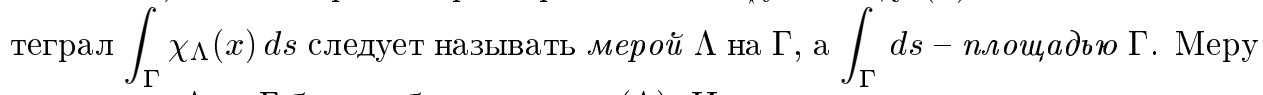
множества $\Lambda$ на $Г$ будем обозначать $m_{\Gamma}(\Lambda)$. Из этого и предыдушего следует, что граница Г липшицевой области имеет п.в. (на Г) касательную плоскость и единичную внешнюю нормаль.

Подмножество $\Lambda$ границы Г называем открытылм, если $\Lambda$ - открытое множество в топологии на $\Gamma$, индуцируемой топологией $\mathbb{R}^{n}$. Через $\partial \Lambda$ обозначаем границу $\Lambda$. В дальнейшем будет использовано также следующее понятие. 
ОПРЕДЕЛЕНИЕ 2.2. Открытое подмножество $\Lambda$ липшицевой границы $Г$ области $\Omega$ будем назьвать липшицевым куском $\Gamma$, если для любой т. $P \in \partial \Lambda$ найдется такая подходящая декартова система координат $\xi=\left(\xi^{\prime \prime}, \xi_{n-1}, \xi_{n}\right)$, $\xi^{\prime \prime}=\left(\xi_{1}, \ldots, \xi_{n-2}\right)$ с центром в т. $P$ и такая окрестность $O(P)=\left\{x \in \mathbb{R}^{n}\right.$ : $\left.\left|\xi^{\prime \prime}\right|<r_{P},\left|\xi_{n-1}\right|<\delta_{P},\left|\xi_{n}\right|<\delta_{P}\right\}, r_{P}>0, \delta_{P}>0$, что наряду с выполнением в определении 2.1 свойств (2.1)-(2.3) с $O^{\prime}(P)=\left\{\xi^{\prime}:\left|\xi^{\prime \prime}\right|<r_{P},\left|\xi_{n-1}\right|<\delta_{P}\right\}$ выполняются еше следующие свойства. Множество $\partial \Lambda \cap O(P)$ имеет в декартовой системе $\xi$ представление

$$
\xi_{n}=F_{P}\left(\xi^{\prime \prime}, \xi_{n-1}\right), \quad \xi_{n-1}=G_{P}\left(\xi^{\prime \prime}\right) \quad \forall \xi^{\prime \prime} \in O^{\prime \prime}(P)=\left\{\xi^{\prime \prime}:\left|\xi^{\prime \prime}\right|<r_{P}\right\},
$$

где $G_{P}\left(\xi^{\prime \prime}\right)$ непрерьвна по Липшицу на $O^{\prime \prime}(P)$ с некоторой постоянной $L_{P}^{\prime}$, и при этом сушествует такое $h_{P}^{\prime}>0$, что множество

$$
\left\{x \in \mathbb{R}^{n}: \xi_{n}=F_{P}\left(\xi^{\prime}\right), G_{P}\left(\xi^{\prime \prime}\right)-h_{P}^{\prime}<\xi_{n-1}<G_{P}\left(\xi^{\prime \prime}\right), \xi^{\prime \prime} \in O^{\prime \prime}(P)\right\}
$$

принадлежит $\Lambda$, а множество

$$
\left\{x \in \mathbb{R}^{n}: \xi_{n}=F_{P}\left(\xi^{\prime}\right), G_{P}\left(\xi^{\prime \prime}\right) \leqslant \xi_{n-1}<G_{P}\left(\xi^{\prime \prime}\right)+h_{P}^{\prime}, \xi^{\prime \prime} \in O^{\prime \prime}(P)\right\}
$$

принадлежит $\Gamma$, но не имеет общих точек с $\Lambda$.

Из этого определения непосредственно вытекает, что если $\Lambda$ - открытый липшицев кусок $\Gamma$, то таковым является и множество $\Gamma \backslash \bar{\Lambda}$.

Примем для дальнейшего следуюшее обозначения

$C(\Omega)$ - пространство функций, непрерьвных в области $\Omega$;

$C^{k}(\Omega), k \geqslant 0$, целое, - подпространство функций $u(x)$ из $C(\Omega)$, для которых существуют все $D^{\alpha} u(x) \in C(\Omega),|\alpha| \leqslant k$, где $D^{\alpha}=D_{1}^{\alpha_{1}} \cdots D_{n}^{\alpha_{n}},|\alpha|=\alpha_{1}+\cdots+\alpha_{n}$, $C^{0}(\Omega)=C(\Omega)$

$C(\bar{\Omega})$ - подпространство функций из $C(\Omega)$, допускающих продолжения на замыкание $\bar{\Omega}$ как непрерывные на $\bar{\Omega}$ функции;

$C^{k}(\bar{\Omega})$ - пространство функций $u(x)$ из $C^{k}(\Omega)$, для которых $D^{\alpha} u(x) \in C(\bar{\Omega}) \forall \alpha$ : $|\alpha| \leqslant k ;$

$$
C^{\infty}(\Omega)=\bigcap_{k=1}^{\infty} C^{k}(\Omega), \quad C^{\infty}(\bar{\Omega})=\bigcap_{k=1}^{\infty} C^{k}(\bar{\Omega}) ;
$$

$C_{\mathbb{R}^{n}}^{\infty}(\bar{\Omega})$ - пространство функций на $\bar{\Omega}$, являюшихся сужениями на $\bar{\Omega}$ функций из $\mathscr{D}\left(\mathbb{R}^{n}\right)$;

$C^{\operatorname{Lip}}(\bar{\Omega})$ - подпространство функций из $C(\bar{\Omega})$, непрерывных по Липшицу в $\bar{\Omega}$ (каждая функция со своей константой Липшица).

Классическая формула Гаусса-Остроградского

$$
\int_{\Omega} \operatorname{div} \boldsymbol{u} d x=\int_{\Gamma}(\boldsymbol{u}, \boldsymbol{n}) d s
$$

заведомо остается справедливой для липшицевой области $\Omega$ и произвольного векторного поля $\boldsymbol{u}(x) \in C^{1}(\bar{\Omega})$ (см. также [26], где эта формула устанавливается в значительно более обших предположениях). 
Пространства Соболева-Слободецкого в области $\Omega$. Ввиду наличия у уравнения (1.3) параметра $\mu$, который может быть большим, естественно, как и в [4], использовать нормировки таких пространств со вхождением параметра $\mu$, имеюшим, грубо говоря, “вес производной”. В нижеследующих определениях будем полагать $\mu>0$.

$W_{2, \mu}^{1}(\Omega)$ - подпространство функций $u(x)$ из $L_{2}(\Omega)$, имеющих все обобщенные первые производные $D_{j} u(x) \in L_{2}(\Omega)$. Скалярное произведение в этом пространстве определяем по формуле

$$
(u, v)_{1, \mu}=\int_{\Omega}\left[(\nabla u(x), \nabla \overline{v(x)})+\mu^{2} u(x) \overline{v(x)}\right] d x,
$$

а норму функции $u(x)$ обозначаем $\|u\|_{W_{2, \mu}^{1}(\Omega)}$;

$W_{2, \mu}^{l}(\Omega)$, где $0<l<1,-$ подпространство функций $u(x)$ из $L_{2}(\Omega)$, для которых конечна норма, определяемая выражением

$$
\|u\|_{W_{2, \mu}^{l}(\Omega)}^{2}=k(n, l) \int_{\Omega} \int_{\Omega} \frac{|u(x)-u(y)|^{2}}{|x-y|^{n+2 l}} d x d y+\mu^{2 l} \int_{\Omega}|u(x)|^{2} d x,
$$

где ради удобства нормировки полагается

$$
k(n, l)=4^{l-1}\left[\int_{|y|=1}|\cos \theta|^{2 l} d s_{y} \int_{0}^{\infty} t^{-(1+2 l)} \sin ^{2} t d t\right]^{-1},
$$

$\theta$ - угол между вектором $y$ и осью $O x_{n}$ (см. [22]). Пространство $W_{2, \mu}^{l}\left(\mathbb{R}^{n}\right), 0<$ $l \leqslant 1$, состоит из тех и только тех функций $u(x) \in L_{2}\left(\mathbb{R}^{n}\right)$, для которых конечна норма

$$
\|\widetilde{u}\|_{L_{2}\left(\mathbb{R}^{n} ;\left(\mu^{2 l}+|\xi|^{2 l}\right)\right)}^{2}=\int_{\mathbb{R}^{n}}\left(\mu^{2 l}+|\xi|^{2 l}\right)|\widetilde{u}(\xi)|^{2} d \xi,
$$

где $\widetilde{u}(\xi)=F[u](\xi)$ - образ Фурье функции $u(x) \in L_{2}\left(\mathbb{R}^{n}\right)$ в пространстве Шварца $\mathscr{S}^{\prime}\left(\mathbb{R}^{n}\right)$ обобшенных функций медленного роста $\left(\right.$ на $\varphi(x) \in \mathscr{S}\left(\mathbb{R}^{n}\right)$ оператор $F$ действует по формуле

$$
F[\varphi](\xi)=\left(\frac{1}{\sqrt{2 \pi}}\right)^{n} \int_{\mathbb{R}^{n}} e^{i(x, \xi)} \varphi(x) d x
$$

см., например, [29]). При этом

$$
\|u\|_{W_{2, \mu}^{l}\left(\mathbb{R}^{n}\right)}=\|\widetilde{u}\|_{L_{2}\left(\mathbb{R}^{n} ;\left(\mu^{2 l}+|\xi|^{2 l}\right)\right)}
$$

Кроме того, пространство $\mathscr{D}\left(\mathbb{R}^{n}\right)$ плотно в $W_{2, \mu}^{l}\left(\mathbb{R}^{n}\right)($ см. [22]).

Заметим, что равенство (2.8) обеспечивается приведенным выше выбором постоянной $k(n, l)$. 
Пространства Соболева-Слободецкого на границе Г липшицевой области $\Omega$. Пусть $\Lambda$ - открытое подмножество $\Gamma$.

$W_{2, \mu}^{l}(\Lambda), 0<l<1,-$ подпространство функций $u(x)$ из $L_{2}(\Lambda)$, для которых конечна норма, определяемая формулой

$$
\|u\|_{W_{2, \mu}^{l}(\Lambda)}^{2}=k(n-1, l) \int_{\Lambda} \int_{\Lambda} \frac{|u(x)-u(y)|^{2}}{|x-y|^{n-1+2 l}} d s_{x} d s_{y}+\mu^{2 l} \int_{\Lambda}|u(x)|^{2} d s_{x}
$$

(см. [21], [9]).

Все пространства $W_{2, \mu}^{l}(\Omega)$ и $W_{2, \mu}^{l}(\Lambda)$ являются полными и при введении для $0<l<1$ в них скалярных произведений, согласованных с нормами (2.7) и (2.9), становятся гильбертовыми пространствами (см. [22]). Кроме того, естественно, нормы каждого из этих пространств, отвечающие различным значениям $\mu$, эквивалентны (но с постоянными зависящими от $\mu$ ). Пространства $W_{2, \mu}^{l}(\Omega)$ и $W_{2, \mu}^{l}(\Lambda)$, отвечающие нормировкам с $\mu=1$, будем обозначать через $W_{2}^{l}(\Omega)$ и $W_{2}^{l}(\Lambda)$. Приведем необходимые нам свойства этих пространств.

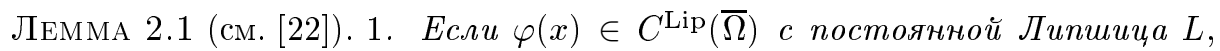
$u(x) \in W_{2, \mu}^{1}(\Omega), \operatorname{mo} \varphi(x) u(x) \in W_{2, \mu}^{1}(\Omega) u$

$$
\|\varphi u\|_{W_{2, \mu}^{1}(\Omega)}^{2} \leqslant 2\left(\max _{x \in \bar{\Omega}}|\varphi(x)|+n L^{2} \mu^{-2}\right)\|u\|_{W_{2, \mu}^{1}(\Omega)}^{2} .
$$

2. Если $\varphi(x)$ - измеримая функиия на $\Lambda \subset \Gamma$, для которой при некотором $l$, $0<l<1$, конечна величина

$$
\frac{1}{2}\|\varphi\|_{A_{2, \mu}^{l}(\Lambda)}^{2}=\underset{x \in \Lambda}{\operatorname{ess} \sup }|\varphi(x)|^{2}+\frac{k(n-1, l)}{\mu^{2 l}} \operatorname{ess~sup}_{x \in \Lambda} \int_{\Lambda} \frac{|\varphi(x)-\varphi(y)|^{2}}{|x-y|^{n-1+2 l}} d s_{y}
$$

$u(x) \in W_{2, \mu}^{l}(\Lambda), \operatorname{mo} \varphi(x) u(x) \in W_{2, \mu}^{l}(\Lambda) u$

$$
\|\varphi u\|_{W_{2, \mu}^{l}(\Lambda)} \leqslant\|\varphi\|_{A_{2, \mu}^{l}(\Lambda)}\|u\|_{W_{2, \mu}^{l}(\Lambda)} .
$$

В частности, если $\varphi(x)$ непрерывна по Липшичу на $\bar{\Lambda}$, то

$$
\|\varphi\|_{A_{2, \mu}^{l}(\Lambda)}<\infty \quad \forall l: 0<l<1 .
$$

ЛЕмма 2.2. Пусть $\Omega$ и $\widetilde{\Omega}$ - области в $\mathbb{R}^{n}, y=y(x)$ - гомеоморфное отображение $\Omega$ на $\widetilde{\Omega}$, являющееся билипшичевым, что обозначает, что и прямое отображение $y=y(x)$, и обратное отображсене $x=x(y)$ являются липиичевими:

$$
\begin{array}{ll}
\left|y\left(x^{1}\right)-y\left(x^{2}\right)\right| \leqslant L\left|x^{1}-x^{2}\right| & \forall x^{1}, x^{2} \in \Omega, \\
\left|x\left(y^{1}\right)-x\left(y^{2}\right)\right| \leqslant \widetilde{L}\left|y^{1}-y^{2}\right| & \forall y^{1}, y^{2} \in \widetilde{\Omega} .
\end{array}
$$

Пространство $W_{2, \mu}^{1}$ инвариантно относительно такого отображения. А именно, если $u(x) \in W_{2, \mu}^{1}(\Omega)$, то $\widetilde{u}(y)=u(x(y)) \in W_{2, \mu}^{1}(\widehat{\Omega})$ и обратно. При 
этом обобщенные производные от $\widetilde{u}(y)$ находятся по классическому правилу вычисления производных от суперпозиции функиий, производные $\partial x_{k} / \partial y_{j}$ понимаются в классическом смысле. Имеют место оценки

$$
\begin{aligned}
& \|\widetilde{u}\|_{W_{2, \mu}^{1}(\widetilde{\Omega})} \leqslant c\|u\|_{W_{2, \mu}^{1}(\Omega)}, \\
& \|u\|_{W_{2, \mu}^{1}(\Omega)} \leqslant \widetilde{c}\|\widetilde{u}\|_{W_{2, \mu}^{1}(\widehat{\Omega})},
\end{aligned}
$$

əде $c=L^{n / 2} \max (1, \widetilde{L}), \widetilde{c}=\widetilde{L}^{n / 2} \max (1, L)$.

По поводу леммы 2.2 см., например, [26], [9]. При доказательстве лемм 2.1 и 2.2 используется определение обобщенной производной, восходящее к Б. Леви (см. [25, гл. 4]).

ТЕОРЕМА 2.1. Пусть $\Omega$ - ограниченная область с липиицевой границей $\Gamma$, $\Lambda$ - открытый липиицев кусок границы Г. Тогда

1) существует линейный оператор $l_{\Omega}^{\mathbb{R}^{n}}$ продолжения функиий $u(x) \in W_{2, \mu}^{l}(\Omega)$ с $\Omega$ на все $\mathbb{R}^{n}$ функциями из $W_{2, \mu}^{l}\left(\mathbb{R}^{n}\right), 0<l \leqslant 1$, и при этом

$$
\left\|l_{\Omega}^{\mathbb{R}^{n}} u\right\|_{W_{2, \mu}^{l}\left(\mathbb{R}^{n}\right)} \leqslant c(\Omega)\|u\|_{W_{2, \mu}^{l}(\Omega)} \quad \forall u \in W_{2, \mu}^{l}(\Omega)
$$

2) существует линейнвй оператор $l_{\Lambda}^{\Gamma}$ продолэсения функиий $u(x) \in W_{2, \mu}^{l}(\Lambda)$ с $\Lambda$ на всю Г функциями из $W_{2, \mu}^{l}(\Gamma), 0<l<1$, и при этом

$$
\left\|l_{\Lambda}^{\Gamma} u\right\|_{W_{2, \mu}^{l}(\Gamma)} \leqslant c(\Lambda, \Gamma)\|u\|_{W_{2, \mu}^{l}(\Lambda)} \quad \forall u \in W_{2, \mu}^{l}(\Lambda) .
$$

Здесь $c(\Omega)$ и с $(\Lambda, \Gamma)$ - постоянные, зависящие только от $\Omega$ и от $\Lambda$ и $\Gamma$, соответственно.

Эти утверждения доказываются аналогично соответствуюшему утверждению типа 1) в [22] с помощью локализации с гладким разбиением единицы, распрямления границ Г и $\partial \Lambda$ и использования “четных" продолжений относительно получаемых плоских участков границ.

Так как липшицева область $\Omega$ удовлетворяет сегментному свойству, то $C_{\mathbb{R}^{n}}^{\infty}(\bar{\Omega})$ плотно в $W_{2, \mu}^{l}(\Omega), l>0$ (см., например, [9], [24], [26]).

ТЕОРЕмА 2.2 (вложения для липшищ-непрерьвных функций). Пусть $\Omega$ - ограниченная область с липшищевой границей $\Gamma, u(x) \in C^{\operatorname{Lip}}(\bar{\Omega})$. Тогда

$$
\begin{gathered}
u(x) \in W_{2}^{l}(\Omega) \quad \forall l: 0<l \leqslant 1, \\
\left.u(x)\right|_{\Gamma} \in W_{2}^{\lambda}(\Gamma) \quad \forall \lambda: 0<\lambda<1,
\end{gathered}
$$

и имеет место оценка

$$
\|u\|_{W_{2, \mu}^{l-1 / 2}(\Gamma)} \leqslant c_{0}(\Omega, l)\|u\|_{W_{2, \mu}^{l}(\Omega)}
$$


$\forall l: 1 / 2<l \leqslant 1$, с постоянной $c_{0}(\Omega, l)$, которая может быть выбрана не зависящей от $\mu$.

Доказательство этой теоремы аналогично доказательству соответствуюшего утверждения в [22] и [4]. С помощью локализации и распрямления границы билипшицевьм отображением оно сводится к установлению такого же утверждения в случае, когда $\Omega$ - полупространство $\mathbb{R}_{-}^{n}=\left\{x \in \mathbb{R}^{n}, x_{n}<0\right\}$, а $u(x)$ имеет, кроме того, конечньй носитель. При этом в отличие от случая, когда $u(x)$ достаточно гладкая, необходимы соответствующие уточнения в обоснованиях при использовании преобразования Фурье.

Определение следа функции $u(x)$ из $W_{2, \mu}^{l}(\Omega), 1 / 2<l \leqslant 1$, примем по схеме, использованной в [22], с приближением $u(x)$ гладкими функциями. Согласно предыдущему для любой $u(x) \in W_{2, \mu}^{l}(\Omega)$ найдется последовательность функций $u_{\nu}(x) \in C^{\operatorname{Lip}}(\bar{\Omega})\left(\right.$ даже $\left.u_{\nu}(x) \in C_{\mathbb{R}^{n}}^{\infty}(\bar{\Omega})\right)$ сходящаяся при $\nu \rightarrow \infty$ в $W_{2, \mu}^{l}(\Omega)$ к $u(x)$. Тогда согласно (2.13) последовательность $\left.u_{\nu}(x)\right|_{\Gamma}$ фундаментальна в $W_{2, \mu}^{l-1 / 2}(\Gamma)$, и в силу полноты последнего пространства существует предельная для этой последовательности функция $u_{*}(x) \in W_{2, \mu}^{l-1 / 2}(\Gamma)$, которая и называется следом на $\Gamma$ функции $u(x)$. Всюду в дальнейшем след функции $u(x) \in W_{2}^{1}(\Omega)$ на открытом куске $\Lambda$ границы $Г$ будем обозначать через $\left.u(x)\right|_{\Lambda}$.

Таким образом, имеет место теорема вложения.

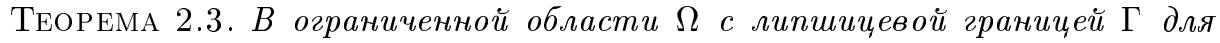
любой функиии $u(x) \in W_{2, \mu}^{l}(\Omega), 1 / 2<l \leqslant 1$, однозначно определен ее след на Г, принадлежсащий пространству $W_{2, \mu}^{l-1 / 2}(\Gamma), u, c$ обозначением этого следа также через $и(x)$, имеет место оценка (2.13). (см. [22], [4], а также [9]).

Имеет место обрашение теоремы 2.3.

ТЕОРЕМА 2.4. Пусть $\Omega$ - ограниченная область с липиицевой границей Г. Существует линейный оператор продолжсения $l_{\Gamma}^{\Omega}$, (зависящий от $\mu$ ), который всякой функиии $u(x) \in W_{2, \mu}^{l-1 / 2}(\Gamma), 1 / 2<l \leqslant 1$, ставит в соответствие функиию $l_{\Gamma}^{\Omega} u(x) \in W_{2, \mu}^{l}(\Omega)$ так, что $\left.l_{\Gamma}^{\Omega} u(x)\right|_{\Gamma}=u(x)$, и имеет место оценка

$$
\left\|l_{\Gamma}^{\Omega} u\right\|_{W_{2, \mu}^{l}(\Omega)} \leqslant c_{1}(\Omega, l)\|u\|_{W_{2, \mu}^{l-1 / 2}(\Gamma)}
$$

с постоянной $c_{1}(\Omega, l)$, которая может быть также выбрана не зависящей om $\mu$.

Доказательство в основном аналогично доказательству соответствуюшего утверждения в [22]. С помошью локализации с применением гладкого разбиения единицы, распрямления границы в подходящих окрестностях задача сводится к продолжению финитной функции $u(x)$ с гиперплоскости $x_{n}=0$ в полупространство $\mathbb{R}_{-}^{n}$. В качестве такого продолжения берется решение $U(x)$ задачи $-\Delta U+\mu^{2} U=0$ в $\mathbb{R}_{-}^{n}, U\left(x^{\prime}, 0\right)=u\left(x^{\prime}\right)$, домноженное на “срезающую” функцию.

В дальнейшем будут использоваться также следующие пространства. В предположениях, что $\Omega$ - ограниченная область с липшицевой границей $\Gamma, \Lambda$ - открытое, $\Gamma_{1}$ - измеримое множества на $Г$ и $\Gamma_{1} \subset \Lambda$, обозначим: 
$W_{2, \mu}^{-1}(\Omega)$ - пространство сопряженное к $W_{2, \mu}^{1}(\Omega)$ (т.е. пространство линейных непрерывных функционалов на $\left.W_{2, \mu}^{1}(\Omega)\right)$;

$\stackrel{\circ}{W} \underset{2, \mu}{1}(\Omega ; \Lambda)$ - подпространство пространства $W_{2, \mu}^{1}(\Omega)$, состоящее из функций $u(x)$, удовлетворяюших условию $\left.u(x)\right|_{\Lambda}=0$;

$\stackrel{\circ}{W}-1, \mu(\Omega ; \Lambda)$ - пространство, сопряженное пространству $\stackrel{\circ}{W} \underset{2, \mu}{1}(\Omega ; \Lambda) ;$

$\stackrel{\circ}{W} \underset{2, \mu}{1 / 2}\left(\Lambda ; \Gamma_{1}\right)$ - подпространство пространства $W_{2, \mu}^{1 / 2}(\Lambda)$ (с той же нормой), состоящее из функций, обращающихся в нуль п.в. на $\Gamma_{1}$;

$\stackrel{\circ}{W} \underset{2, \mu}{-1 / 2}\left(\Lambda ; \Gamma_{1}\right)$ - пространство, сопряженное пространству $\stackrel{\circ}{W} \underset{2, \mu}{1 / 2}\left(\Lambda ; \Gamma_{1}\right) ;$

в случае, когда $\Lambda=\Gamma$, а $\Gamma_{2}$ - открытое множество на $\Gamma$, для сокрашения будем обозначать $\stackrel{\circ}{W} \underset{2, \mu}{1 / 2}\left(\Gamma_{2}\right)=\stackrel{\circ}{W} \underset{2, \mu}{1 / 2}\left(\Gamma ; \Gamma \backslash \Gamma_{2}\right)$, через $\stackrel{\circ}{W} \underset{2, \mu}{-1 / 2}\left(\Gamma_{2}\right)$ - пространство, сопряженное к $\stackrel{\circ}{W} \underset{2, \mu}{1 / 2}\left(\Gamma_{2}\right)$.

При $\Lambda=\varnothing \stackrel{\circ}{W_{2, \mu}^{ \pm 1}}(\Omega ; \Lambda)=W_{2, \mu}^{ \pm 1}(\Omega)$, при $\Gamma_{2}=\Gamma \stackrel{\circ}{W_{2, \mu}^{ \pm 1 / 2}}\left(\Gamma_{2}\right)=W_{2, \mu}^{ \pm 1 / 2}(\Gamma)$. Очевидно, что $\stackrel{\circ}{W} \frac{1}{2, \mu}(\Omega ; \Lambda)$ и $\stackrel{\circ}{W} \underset{2, \mu}{1 / 2}\left(\Lambda ; \Gamma_{1}\right)$ - замкнутые подпространства пространств $W_{2, \mu}^{1}(\Omega)$ и $W_{2, \mu}^{1 / 2}(\Lambda)$, соответственно.

ТЕОРема 2.5. Операторы вложсения пространства $W_{2}^{l}(\Omega), 0<l \leqslant 1$, в $L_{2}(\Omega)$ и пространства $W_{2}^{l}(\Gamma), 0<l \leqslant 1$, в $L_{2}(\Gamma)$ являются вполне непрерывными операторами.

Эти утверждения устанавливаются аналогично соответствующим утверждениям для пространств $W_{2}^{l}(\Omega)$, например, в [20], [24]-[26], [18] (случай $l=1$ ) и в [22] (когда $l$ нецелое, с соответствующими уточнениями там формулировок леммы 8 и теоремы $11 \S 7)$.

Нам понадобится еще следующее утверждение.

ЛЕМма 2.3. В предположсениях, что $\Omega$-ограниченная область с липиицевой границей $\Gamma, \Lambda$ - открытый липшищев кусок $\Gamma$, в пространстве $\stackrel{\circ}{W} \underset{2}{1}(\Omega ; \Lambda)$ плотно мнохсество функиий $\left\{u(x) \in C_{\mathbb{R}^{n}}^{\infty}(\bar{\Omega}),\left.u(x)\right|_{\Lambda}=0\right\}$.

ДоказАТЕльство. Сначала установим следующую лемму.

Лемма 2.4. Пусть $u(x) \in \stackrel{\circ}{W} \underset{2}{1}(\Omega ; \Lambda)$. Тогда функиия

$$
\stackrel{\circ}{u}(x)= \begin{cases}u(x) & \text { nрu } x \in \Omega, \\ 0 & \text { npu } x \in\left(\mathbb{R}^{n} \backslash \bar{\Omega}\right) \cup \Lambda\end{cases}
$$

принадлежит пространству $W_{2}^{1}\left(\mathbb{R}^{n} \backslash(\Gamma \backslash \Lambda)\right)$, и норма $\stackrel{\circ}{u}(x)$ в этом пространстве совпадает $c\|u\|_{W_{2}^{1}(\Omega)}$.

ДоКАЗАТЕЛЬСТво ЛЕмМы 2.4. В силу плотности $C^{\infty}(\bar{\Omega})$ в $W_{2}^{1}(\Omega)$ сушествует последовательность $u_{\nu}(x) \in C^{\infty}(\bar{\Omega})$ такая, что $u_{\nu}(x) \rightarrow u(x)$ в $W_{2}^{1}(\Omega)$ при $\nu \rightarrow \infty$. Тогда в силу определения следа и теоремы $2.2 u_{\nu} \rightarrow 0$ в $W_{2}^{1 / 2}(\Lambda)$ при $\nu \rightarrow \infty$. 
Пусть теперь $\varphi(x) \in \mathscr{D}\left(\mathbb{R}^{n} \backslash(\Gamma \backslash \Lambda)\right)$ - произвольная. Тогда, используя формулу Гаусса-Остроградского, получим

$$
\begin{aligned}
-\int_{\mathbb{R}^{n} \backslash(\Gamma \backslash \Lambda)} & \stackrel{\circ}{u}(x) D_{k} \varphi(x) d x=-\lim _{\nu \rightarrow \infty} \int_{\Omega} u_{\nu}(x) D_{k} \varphi(x) d x \\
= & \lim _{\nu \rightarrow \infty} \int_{\Omega} D_{k} u_{\nu}(x) \varphi(x) d x-\lim _{\nu \rightarrow \infty} \int_{\Lambda} u_{\nu}(x) n_{k}(x) \varphi(x) d s_{x} \\
= & \int_{\Omega} D_{k} u(x) \varphi(x) d x=\int_{\mathbb{R}^{n}}\left(D_{k} u(x)\right)^{\circ} \varphi(x) d x,
\end{aligned}
$$

поскольку

$$
\left|\int_{\Lambda} u_{\nu}(x) n_{k}(x) \varphi(x) d x\right| \leqslant\left\|u_{\nu}\right\|_{L_{2}(\Lambda)}\|\varphi\|_{L_{2}(\Gamma)} \rightarrow 0 \quad \text { при } \quad \nu \rightarrow \infty .
$$

Таким образом, $D_{k} \stackrel{\circ}{u}(x)=\left(D_{k} u(x)\right)^{\circ}$ в смысле обобщенных функций.

Перейдем к доказательству леммы 2.3. Применим стандартную для доказательства такого рода утверждений технику (см., например, [24, гл. III]), начинаюшуюся с рассмотрения достаточно мелкого покрытия компакта $\bar{\Omega}$ окрестностями, (причем специального, приводимого ниже вида для граничных окрестностей), выделения из такого покрытия конечного, умножения функции $\stackrel{\circ}{u}(x)$, появляющейся в лемме 2.4 на $1 \equiv \sum_{j} \psi_{j}(x)$, где $\psi_{j}(x)$ - конечное гладкое разбиение единицы, подчиненное выделенному покрытию. В результате $u(x)=\sum_{j} u_{j}(x)$, где $u_{j}(x)=\psi_{j}(x) u(x) \in W_{2}^{1}(\Omega)$, и доказательство леммы 2.3 может быть сведено к доказательству ее в следующих случаях.

1) $\operatorname{supp} u(x) \subset \Omega$.

2) Носитель $u(x)$ содержится в окрестности $U_{P}\left(h_{P} / 2, r_{P} / 2\right)$ точки $P \in \Gamma \backslash \partial \Lambda$, где $U_{P}\left(h_{P}, r_{P}\right)$ - объединение множеств $(2.3 \mathrm{a})$ и $(2.3 б)$, причем $r_{P}>0$ столь мало, что:

a) $U_{P}\left(h_{P}, r_{P}\right) \cap(\Gamma \backslash \Lambda)=\varnothing$ в том случае, когда $P \in \Lambda$;

б) $U_{P}\left(h_{P}, r_{P}\right) \cap \bar{\Lambda}=\varnothing$ в том случае, когда $P \in \Gamma \backslash \bar{\Lambda}$.

$3)$ Носитель $u(x)$ расположен в окрестности $U_{P}\left(h_{P} / 2, h_{P}^{\prime} / 2, r_{P} / 2\right)$ точки $P \in$ $\partial \Lambda$, где

$$
\begin{aligned}
U_{P}\left(h_{P}, h_{P}^{\prime}, r_{P}\right)= & \left\{x \in \mathbb{R}^{n}: \xi \in \widetilde{U}_{P}\left(h_{P}, h_{p}^{\prime}, r_{P}\right)\right\}, \\
\widetilde{U}_{P}\left(h_{P}, h_{P}^{\prime}, r_{P}\right)=\left\{\xi:\left|\xi_{n}-F_{P}\left(\xi^{\prime}\right)\right|<h_{P},\right. & \left.\left|\xi_{n-1}-G_{P}\left(\xi^{\prime \prime}\right)\right|<h_{P}^{\prime},\left|\xi^{\prime \prime}\right|<r_{P}\right\},
\end{aligned}
$$

$\xi$ - подходящая декартова система координат, соответствующая точке $P, F_{P}\left(\xi^{\prime}\right)$ и $G_{P}\left(\xi^{\prime \prime}\right)$ - функции, $h_{P}, h_{P}^{\prime}$ и $r_{P}$ - величины, фигурирующие в определении 2.2 .

В каждом из выделенных случаев нужно для $u(x)$ построить последовательность функций $u_{\nu}(x) \in C_{\mathbb{R}^{n}}^{\infty}(\bar{\Omega})$ таких, что $\left.u_{\nu}(x)\right|_{\Lambda}=0$ и $u_{\nu}(x) \rightarrow u(x)$ в $W_{2}^{1}(\Omega)$ при $\nu \rightarrow \infty$. В случаях 1) и 2) это делается обычным образом с помощью операторов усреднения. В случае 1$)$ такой последовательностью является $u_{\nu}(x)=J_{\varepsilon_{\nu}} u(x)$, 
$0<\varepsilon_{\nu}<d, \varepsilon_{\nu} \rightarrow 0$ при $\nu \rightarrow \infty$, где $J_{\varepsilon}-$ оператор усреднения Соболева с радиусом усреднения $\varepsilon$ (см., например, [20], [24], [25]), $d=\operatorname{dist}\left\{\operatorname{supp} u, \mathbb{R}^{n} \backslash \Omega\right\}$. В случае $2 \sigma$ ) $u(x)$ продолжается в окрестность $U_{P}\left(h_{P} / 2, r_{P} / 2\right)$, а затем уже нулем на все пространство как функция $\widehat{u}(x) \in W_{2}^{1}\left(\mathbb{R}^{n}\right)$ (такое продолжение можно осуществить “четньм продолжением" через участок границы $\Gamma \cap U_{P}\left(h_{P}, r_{P}\right)$ при распрямлении этого участка границы), и последовательность $u_{\nu}(x)=\left.J_{\varepsilon_{\nu}} \widehat{u}(x)\right|_{\Omega}$, где $\varepsilon_{\nu}$ достаточно малые, $\varepsilon_{\nu} \rightarrow 0$, обладает нужными свойствами. В случае $2 \mathrm{a}$ ) сначала строится последовательность $\stackrel{o}{u}_{\nu}^{\prime}(x)=\stackrel{\circ}{u}\left(x^{\prime}+\delta_{\nu} e_{P}\right)$, где $0<\delta_{\nu}<h_{P} / 2, e_{P}$ - единичный вектор, имеющий в подходящей декартовой системе $\xi$ для т. $P$ координаты $(0, \ldots, 0,1), \stackrel{\circ}{u}(x)$ - функция, фигурируюшая в лемме 2.4 , а затем - последовательность $u_{\nu}(x)=J_{\varepsilon_{\nu}} \stackrel{\circ}{u}_{\nu}^{\prime}(x)$, где $0<\varepsilon_{\nu}<\operatorname{dist}\left\{\operatorname{supp} \stackrel{\circ}{u}_{\nu}^{\prime}, \mathbb{R}^{n} \backslash \Omega\right\}, \varepsilon_{\nu} \rightarrow 0$ при $\nu \rightarrow \infty$, которая обладает нужньми свойствами $\left(u_{\nu}(x) \in \mathscr{D}(\Omega)\right)$.

Наконец, рассмотрим случай 3$)$. Обозначим через $\stackrel{\circ}{u}(\xi)=\stackrel{\circ}{u}\left(\xi^{\prime}, \xi_{n}\right)$ выражение в подходящей декартовой системе координат $\xi$ для т. $P$ функции $\stackrel{\circ}{u}(x)$, появляющейся в лемме 2.4. Для сокращения примем также следующие обозначения:

$$
\begin{gathered}
U_{P, \varkappa}=U_{P}\left(\varkappa h_{P}, \varkappa h_{P}^{\prime}, \varkappa r_{P}\right), \quad \widetilde{U}_{P, \varkappa}=\widetilde{U}_{P}\left(\varkappa h_{P}, \varkappa h_{P}^{\prime}, \varkappa r_{P}\right), \\
\varkappa>0, \quad U_{P}=U_{P, 1}, \quad \widetilde{U}_{P}=\widetilde{U}_{P, 1},
\end{gathered}
$$

где $U_{P}\left(h_{P}, h_{P}^{\prime}, r_{P}\right)$ и $\widetilde{U}_{P}\left(h_{P}, h_{P}^{\prime}, r_{P}\right)$ определены $(2.15 \mathrm{a})$ и $(2.15 б)$,

$$
\Gamma_{-}=\Lambda \cap U_{P}, \quad \Gamma_{+}=(\Gamma \backslash \Lambda) \cap U_{P},
$$

$\widetilde{\Gamma}_{-}$и $\widetilde{\Gamma}_{+}-$образы кусков $\Gamma_{-}$и $\Gamma_{+}$в подходящей системе $\xi$. В силу леммы $2.4 \stackrel{\circ}{u}(\xi) \in$ $W_{2}^{1}\left(\widetilde{U}_{P} \backslash \widetilde{\Gamma}_{+}\right)$.

Произведем распрямление границ билипшицевым отображением

$$
\eta^{\prime \prime}=\xi^{\prime \prime}, \quad \eta_{n-1}=\xi_{n-1}-G_{P}\left(\xi^{\prime \prime}\right), \quad \eta_{n}=\xi_{n}-F_{P}\left(\xi^{\prime}\right),
$$

которое переводит окрестность $\widetilde{U}_{P}$ на цилиндрическую окрестность $Q_{1}$, где

$$
Q_{\varkappa}=\left\{\eta:\left|\eta^{\prime \prime}\right|<\varkappa r_{P},\left|\eta_{n-1}\right|<\varkappa h_{P}^{\prime},\left|\eta_{n}\right|<\varkappa r_{P}\right\}, \quad \varkappa>0 .
$$

При этом $\widetilde{\Gamma}_{+}$и $\widetilde{\Gamma}_{-}$переходят, соответственно, в части $\Sigma_{+}$и $\Sigma_{-}$гиперплоскости $\eta_{n}=0$, где

$$
\begin{aligned}
& \Sigma_{+}=\left\{\eta: \eta_{n}=0,0 \leqslant \eta_{n-1}<h_{P}^{\prime},\left|\eta^{\prime \prime}\right|<r_{P}\right\}, \\
& \Sigma_{-}=\left\{\eta: \eta_{n}=0,-h_{P}^{\prime}<\eta_{n-1}<0,\left|\eta^{\prime \prime}\right|<r_{P}\right\} .
\end{aligned}
$$

Функцию, получаюшуюся из $\stackrel{\circ}{u}(\xi)$ при переходе к новым переменным $(2.16)$, обозначим через $\stackrel{\circ}{v}(\eta)$. В силу леммы $2.2 \stackrel{\circ}{v}(\eta) \in W_{2}^{1}\left(Q_{1} \backslash \Sigma_{+}\right)$и, кроме того, $\operatorname{supp} \stackrel{\circ}{v}(\eta) \subset$ $Q_{1 / 2}, \stackrel{\circ}{v}(\eta)=0$, на множестве $Q_{1}^{+}=Q_{1} \cap\left\{\eta: \eta_{n}>0\right\}$; на множестве $Q_{1}^{-}=Q_{1} \cap$ $\left\{\eta: \eta_{n}<0\right\} \stackrel{\circ}{v}(\eta)$ является выражением функции $\left.u(x)\right|_{U_{P} \cap \Omega}$ в переменных $(2.16)$. 
Построим продолжение $\widehat{v}(\eta)$ функции $\left.\stackrel{\circ}{v}(\eta)\right|_{Q_{1}^{-}}$на все пространство $\mathbb{R}^{n}$ такое, чтобы $\widehat{v}(\eta) \in W_{2}^{1}\left(\mathbb{R}^{n}\right), \operatorname{supp} \widehat{v}(\eta) \subset Q_{3 / 4}$, и чтобы $\widehat{v}(\eta)=0$ на некоторой подобласти $M_{0}$ в $Q_{1}^{+}$, примыкаюшей к куску $\Sigma_{-}$. Для этого продолжим $\stackrel{\circ}{v}(\eta)$ нулем вне $Q_{1}$ и используем билипшицев гомеоморфизм

$$
\zeta^{\prime \prime}=\eta^{\prime \prime}, \quad \zeta_{n-1}=\frac{1}{\sqrt{2}}\left(\eta_{n-1}+\left|\eta_{n-1}\right|-\eta_{n}\right), \quad \zeta_{n}=\frac{1}{\sqrt{2}}\left(\eta_{n-1}-\left|\eta_{n-1}\right|+\eta_{n}\right)
$$

$\mathbb{R}^{n}$ на $\mathbb{R}^{n} ;$ обратное отображение действует по формулам

$$
\eta^{\prime \prime}=\zeta^{\prime \prime}, \quad \eta_{n-1}=\frac{1}{\sqrt{2}}\left(\zeta_{n}+\zeta_{n-1}\right), \quad \eta_{n}=\frac{1}{\sqrt{2}}\left(\zeta_{n}-\zeta_{n-1}+\left|\zeta_{n}+\zeta_{n-1}\right|\right)
$$

Обозначим через $\widetilde{v}(\zeta)$ функцию $\stackrel{\circ}{v}(\eta)$, выраженную в переменных $(2.18 \mathrm{a})$, через $\widetilde{Q}_{1}^{-}$ - образ множества $Q_{1}^{-}$при отображении (2.18a).

Поскольку это отображение представляет собой билипшицев гомеоморфизм множества $\mathbb{R}^{n} \backslash\left\{\eta: \eta_{n}=0, \eta_{n-1} \geqslant 0\right\}$ на себя, то в силу леммы 2.2 $\widetilde{v}(\zeta) \in W_{2}^{1}\left(\mathbb{R}_{-}^{n}\right)$, где $\mathbb{R}_{-}^{n}=\left\{\zeta: \zeta_{n}<0\right\}$. Далее, $\widetilde{Q}_{1}^{-}$содержится в четверти пространства $\left\{\zeta: \zeta_{n}<0, \zeta_{n-1}>0\right\}$, а потому $\widetilde{v}(\zeta)$ обращается в нуль на множестве $\left\{\zeta: \zeta_{n}<0, \zeta_{n-1} \leqslant 0\right\}$. Обозначим теперь через $\widetilde{v}_{1}(\zeta)$ четное по переменной $\zeta_{n}$ продолжение функции $\widetilde{v}(\zeta)$ с $\mathbb{R}_{-}^{n}$ на все $\mathbb{R}^{n}$ :

$$
\widetilde{v}_{1}(\zeta)= \begin{cases}\widetilde{v}(\zeta) & \text { при } \zeta_{n}<0 \\ \widetilde{v}\left(\zeta^{\prime},-\zeta_{n}\right) & \text { при } \zeta_{n}>0\end{cases}
$$

(на гиперплоскости $\zeta_{n}=0 \quad \widetilde{v}_{1}(\zeta)$ можно определить как угодно). Тогда $\widetilde{v}_{1}(\zeta) \in W_{2}^{1}\left(\mathbb{R}^{n}\right)$ и $\widetilde{v}_{1}(\zeta)=0$ п.в. в полупространстве $\left\{\zeta: \zeta_{n-1}<0\right\}$.

Это полупространство переводится отображением (2.18б) на область $M_{0}=\{\eta$ : $\left.\eta_{n-1}<\eta_{n} / 2, \eta_{n}>0\right\}$, а потому функция $v_{1}(\eta)$, определяемая как выражение функции $\widetilde{v}_{1}(\zeta)$ в переменных $(2.18 \sigma)$, обращается в нуль на $M_{0}$. Более того, $v_{1}(\eta)=$ $\stackrel{\circ}{v}(\eta)$ на $\mathbb{R}_{-}^{n}$, а потому $v_{1}(\eta)=0$ при $\eta \in \mathbb{R}_{-}^{n} \backslash Q_{1 / 2}$. Пусть теперь $\varphi(\eta) \in \mathscr{D}\left(\mathbb{R}^{n}\right)$, $\varphi(\eta) \equiv 1$ на $Q_{1 / 2}$ и $\varphi(\eta) \equiv 0$ на $\mathbb{R}^{n} \backslash Q_{3 / 4}$. Тогда функция $\widehat{v}(\eta)=\varphi(\eta) v_{1}(\eta)$ и будет необходимьм продолжением функции $\left.\stackrel{\circ}{v}(\eta)\right|_{Q_{1}^{-}}$. Она обладает следующими свойствами: $\widehat{v}(\eta) \in W_{2}^{1}\left(\mathbb{R}^{n}\right), \widehat{v}(\eta)=\stackrel{\circ}{v}(\eta) \forall \eta \in Q_{1}^{-}, \operatorname{supp} \widehat{v}(\eta) \subset Q_{3 / 4}$ и $\widehat{v}(\eta)=0$ $\forall \eta \in M_{0}$.

Образуем теперь последовательность функций $\widehat{v}_{\nu}^{\prime}(\eta)=\widehat{v}\left(\eta^{\prime}, \eta_{n}+\delta_{\nu}\right), 0<\delta_{\nu}<$ $h_{P} / 8, \delta_{\nu} \rightarrow 0$ при $\nu \rightarrow \infty$. Тогда $\widehat{v}_{\nu}^{\prime}(\eta) \rightarrow \widehat{v}(\eta)$ в $W_{2}^{1}\left(\mathbb{R}^{n}\right)$ при $\nu \rightarrow \infty, \operatorname{supp} \widehat{v}_{\nu}^{\prime} \subset$ $Q_{7 / 8}$ и $\widehat{v}_{\nu}^{\prime}(\eta)=0 \forall \eta \in M_{0}-\delta_{\nu} e_{n}$, где $e_{n}-$ вектор с координатами $(0, \ldots, 0,1)$. Отсюда каждая функция $\widehat{v}_{\nu}^{\prime}(\eta)$ обращается в нуль в некоторой окрестности множества $\Sigma_{-}$. Таким образом, последовательность функций $\widehat{u}_{\nu}^{\prime}(x)$, получаемая из функций $\left.\widehat{v}_{\nu}^{\prime}(\eta)\right|_{Q_{1}}$ выражением последних в переменных $x$ и с продолжением их нулем вне множества (2.15a), обладают следующими свойствами: $\widehat{u}_{\nu}^{\prime}(x) \rightarrow \widehat{u}(x)$ в $W_{2}^{1}\left(\mathbb{R}^{n}\right)$ при $\nu \rightarrow \infty$, где $\widehat{u}(x)$ строится по функции $\widehat{v}(\eta)$ точно таким же образом, как $\widehat{u}_{\nu}^{\prime}(x)$ строятся по $\widehat{v}_{\nu}^{\prime}(\eta), \widehat{u}_{\nu}^{\prime}(x)=0$ вне $U_{P, 7 / 8}$, и в некоторой окрестности $\Lambda^{2 \varepsilon_{\nu}}$ куска $\Lambda$, где $\Lambda^{\varepsilon}-\varepsilon$-расширение множества $\Lambda, \varepsilon_{\nu}>0, \varepsilon_{\nu} \rightarrow 0$ при $\nu \rightarrow \infty$. 
Поэтому последовательность $u_{\nu}(x)=J_{\varepsilon_{\nu}} \widehat{u}_{\nu}^{\prime}(x)$, где $J_{\varepsilon}$ - упоминавшийся в начале доказательства оператор усреднения с радиусом усреднения $\varepsilon>0$, обладает нужными свойствами: $u_{\nu}(x) \in \mathscr{D}\left(\mathbb{R}^{n}\right), u_{\nu}(x) \rightarrow \widehat{u}(x)$ в $W_{2}^{1}\left(\mathbb{R}^{n}\right)$ при $\nu \rightarrow \infty$ (здесь используется равномерная ограниченность по $\varepsilon$ норм семейства операторов $\left.J_{\varepsilon}\right)$, а поскольку $\widehat{u}(x)=u(x)$ при $x \in \Omega$, то и $u_{\nu}(x) \rightarrow u(x)$ в $W_{2}^{1}(\Omega)$ при $\nu \rightarrow \infty$, и, наконец, $\left.u_{\nu}(x)\right|_{\Lambda}=0$.

Это и заканчивает доказательство леммы 2.3.

\section{§3. Смешанная задача в липшицевых областях.}

\section{О значении конормальной производной обобщенных решений}

Схема доказательства теорем существования обобщенных решений вариационных задач, основанная на использовании на главном этапе теоремы Рисса о представлении линейного ограниченного функционала в гильбертовом пространстве и сведении затем задачи с младшими членами в уравнении и граничном условии к уравнению Фредгольма второго рода, общеизвестна (см., например, [20], [7], [17]-[20], [9]; в перечисленных руководствах она в основном реализована для краевых задач Дирихле, Неймана или третьего рода). В этом параграфе для полноты изложения приводятся формулировка и доказательство теоремы о фредгольмовости смешанной задачи $(1.1),(1.2)$ с (1.3), (1.4) и сопряженной к ней. В общем случае условия на коэффициенты те же, что и в начале $\S 1: a_{i j}(x), a_{i}(x), a_{0}(x), q(x) \in L_{\infty}(\Omega), \sigma(x) \in L_{\infty}\left(\Gamma_{2}\right)$ и выполняются условия (1.5) и (1.6); относительно $\Gamma_{1}$ и $\Gamma_{2}$ предполагается, что они являются лишь открытыми на $\Gamma$ множествами, в следуюшем смысле составляющими $\Gamma$ : $\Gamma_{1} \cap \Gamma_{2}=\varnothing, m_{\Gamma}\left(\Gamma \backslash\left(\Gamma_{1} \cup \Gamma_{2}\right)\right)=0$. Последнее означает, что $\Gamma=\Gamma_{1} \cup \Gamma_{2} \cup \delta$, где $\Gamma_{1}, \Gamma_{2}$ и $\delta$ попарно не пересекаются и $m_{\Gamma}(\delta)=0$.

Предварительно дадим формальное определение задачи, сопряженной к исходной, и определение обобшенных решений этих задач. Для этого вначале в предположениях достаточной гладкости коэффициентов $a_{i j}(x), a_{i}(x)$ (например, принадлежности пространству $\left.C^{1}(\bar{\Omega})\right)$ и функций $u(x)$ и $v(x)$ (например, принадлежности пространству $C^{2}(\bar{\Omega})$ ) приведем формулы Грина (две - первые и одну - вторую). Для дальнейшего будет удобно представить $a_{0}(x)=a_{0}^{1}(x)+a_{0}^{2}(x)$ и $\sigma(x)=\sigma_{1}(x)+\sigma_{2}(x)$, где $a_{0}^{1}(x) \in L_{\infty}(\Omega)$ и $\sigma_{1}(x) \in L_{\infty}\left(\Gamma_{2}\right)$ - действительные функции (такие представления, естественно, неоднозначны).

Итак, при достаточно гладких коэффициентах и функциях

$$
\begin{aligned}
\int_{\Omega} L(x, D, \mu) u \bar{v} d x= & a[u, v]_{\mu}+\int_{\Omega} \alpha(x, D) u \bar{v} d x \\
& +\int_{\Gamma_{2}} \sigma_{2} u \bar{v} d s-\int_{\Gamma_{1}} \frac{\partial u}{\partial \nu} \bar{v} d s-\int_{\Gamma_{2}}\left(\frac{\partial u}{\partial \nu}+\sigma u\right) \bar{v} d s \\
\int_{\Omega} L^{*}(x, D, \mu) u \bar{v} d x= & a[u, v]_{\bar{\mu}}+\int_{\Omega} u \overline{\alpha(x, D) v} d x \\
& +\int_{\Gamma_{2}} u \overline{\sigma_{2} v} d s-\int_{\Gamma_{1}}\left(\frac{\partial u}{\partial \nu}+\sum_{i=1}^{n} \bar{a}_{i} n_{i} u\right) \bar{v} d s
\end{aligned}
$$




$$
\begin{gathered}
-\int_{\Gamma_{2}}\left[\frac{\partial u}{\partial \nu}+\left(\bar{\sigma}+\sum_{i=1}^{n} \bar{a}_{i} n_{i}\right) u\right] \bar{v} d s \\
\int_{\Omega} L(x, D, \mu) u \bar{v} d x-\int_{\Gamma_{1}} u \frac{\partial \bar{v}}{\partial \nu} d s+\int_{\Gamma_{2}}\left(\frac{\partial u}{\partial \nu}+\sigma u\right) \bar{v} d s \\
=\int_{\Omega} u \overline{L^{*}(x, D, \mu) v} d x-\int_{\Gamma_{1}}\left(\frac{\partial u}{\partial \nu}-\sum_{i=1}^{n} a_{i} n_{i} u\right) \bar{v} d s \\
+\int_{\Gamma_{2}} u \overline{\left[\frac{\partial u}{\partial \nu}+\left(\bar{\sigma}+\sum_{i=1}^{n} \bar{a}_{i} n_{i}\right) v\right]} d s
\end{gathered}
$$

где

$$
\begin{gathered}
a[u, v]_{\mu}=a(u, v)_{\mu}+\int_{\Omega} a_{0}^{1}(x) u(x) \bar{v}(x) d x+\int_{\Gamma_{2}} \sigma_{1}(x) u(x) \bar{v}(x) d s, \\
a(u, v)_{\mu}=\int_{\Omega}\left(\sum_{i, j=1}^{n} a_{i j}(x) D_{j} u(x) D_{i} \bar{v}(x)+\mu^{2} q(x) u(x) \bar{v}(x)\right) d x, \\
\alpha(x, D) u(x)=\sum_{i=1}^{n} a_{i}(x) D_{i} u(x)+a_{0}^{2}(x) u(x), \\
L^{*}(x, D, \mu) u(x)=-\sum_{i, j=1}^{n} D_{i}\left(a_{i j}(x) D_{j} u(x)\right) \\
-\sum_{i=1}^{n} D_{i}\left(\bar{a}_{i}(x) u(x)\right)+\left(\bar{a}_{0}(x)+\bar{\mu}^{2} q(x)\right) u(x),
\end{gathered}
$$

$\partial / \partial \nu$ - операция (1.4) вычисления конормальной производной, $n_{i}=n_{i}(x)-$ компоненты единичной внешней нормали к $\Gamma$.

Исходя из формулы (3.1в), задача, сопряженная к задаче (1.1), (1.2), определяется как смешанная задача

$$
\begin{aligned}
& L^{*}(x, D, \mu) u(x)=f(x) \text { в } \Omega \\
& \left.u(x)\right|_{\Gamma_{1}}=\varphi(x),\left.\quad\left(\frac{\partial u}{\partial \nu}(x)+\left(\bar{\sigma}(x)+\sum_{i=1}^{n} \bar{a}_{i}(x) n_{i}(x)\right) u(x)\right)\right|_{\Gamma_{2}}=g(x) .
\end{aligned}
$$

Определения же обобшенных решений задач (1.1), (1.2) и (3.3), (3.4) даются по обычной схеме на основании интегральных соотношений (3.1а) и (3.1б).

ОПРЕДЕЛЕНИЕ 3.1. Пусть коэффициенты $a_{i j}(x), a_{i}(x), a_{0}(x), q(x)$ и $\sigma(x)$ удовлетворяют условиям, сформулированньм в начале этого параграфа, и пусть в (1.1), (1.2) или (3.3), (3.4) $f(x) \in \stackrel{\circ}{W_{2}^{-1}}\left(\Omega ; \Gamma_{1}\right), \varphi(x) \in W_{2}^{1 / 2}\left(\Gamma_{1}\right), g(x) \in \stackrel{\circ}{W_{2}^{-1 / 2}}\left(\Gamma_{2}\right)$. Под обобщенным решением (о.р.) задачи (1.1), (1.2) или задачи (3.3), (3.4) понимается функция $u(x) \in W_{2}^{1}(\Omega)$, удовлетворяюшая граничному условию $\left.u(x)\right|_{\Gamma_{1}}=$ $\varphi(x)$ в смысле следов, и для любой функции $v(x) \in \stackrel{\circ}{W} \underset{2}{1}\left(\Omega ; \Gamma_{1}\right)-$ соотношению

$$
a[u, v]_{\mu}+\int_{\Omega} \alpha(x, D) u \bar{v} d x+\int_{\Gamma_{2}} \sigma_{2} u \bar{v} d s=\langle f, \bar{v}\rangle_{\Omega}+\langle g, \bar{v}\rangle_{\Gamma_{2}}
$$


для задачи (1.1), (1.2) или соотношению

$$
a[u, v]_{\bar{\mu}}+\int_{\Omega} u \overline{\alpha(x, D) v} d x+\int_{\Gamma_{2}} u \overline{\sigma_{2} v} d s=\langle f, \bar{v}\rangle_{\Omega}+\langle g, \bar{v}\rangle_{\Gamma_{2}}
$$

для задачи (3.3), (3.4). Здесь и далее через $\langle f, v\rangle_{\Omega}$ и $\langle g, v\rangle_{\Gamma_{2}}$ обозначаем действия функционалов $f(x)$ и $g(x)$ на $v(x)$ и на $\left.v(x)\right|_{\Gamma_{2}}$, соответственно.

Отметим, что в этих определениях никак не участвуют граничные значения функций $a_{i j}(x)$ и $a_{i}(x)$, что и позволяет распространить определения обобщенных решений на случай уравнений с измеримыми ограниченными коэффициентами. При этом выражения (1.4) и $\left.\sum_{i=1}^{n} \bar{a}_{i}(x) n_{i}(x)\right|_{\Gamma_{2}}$ в (3.4) в общем носят уже символический характер.

Прежде чем перейти к теореме о разрешимости задач, обсудим понятие конормальной производной на Г от обобшенных решений сформулированных задач. Здесь мы сталкиваемся с невозможностью однозначного определения $\partial u / \partial \nu$ от о.р. и на основе данных, принадлежащих только пространствам, фигурирующим в определении 3.1. Исходя из классических формул (3.1а) и (3.1б), естественно дать следуюшее определение.

ОПРЕДЕЛЕНИЕ 3.2. Пусть $u(x)$ - o.p. задачи $(1.1),(1.2)$ или задачи $(3.3),(3.4)$ c $f(x) \in \stackrel{\circ}{W}-1\left(\Omega ; \Gamma_{1}\right), \varphi(x) \in W_{2}^{1 / 2}\left(\Gamma_{1}\right), g(x) \in \stackrel{\circ}{W_{2}^{-1 / 2}}\left(\Gamma_{2}\right)$, a $\widehat{f} \in W_{2}^{-1}(\Omega)-$ некоторое продолжение функционала $f(x)$ с $\stackrel{\circ}{W} \underset{2}{1}\left(\Omega ; \Gamma_{1}\right)$ на $W_{2}^{1}(\Omega)$. Обозначим

$$
\sigma^{0}(x)= \begin{cases}\sigma(x) & \text { при } x \in \Gamma_{2}, \\ 0 & \text { при } x \in \Gamma \backslash \Gamma_{2} .\end{cases}
$$

Под соответствуюшим продолжению $\widehat{f}$ значением

$$
\left.\left[\frac{\partial u}{\partial \nu}+\sigma^{0} u\right]\right|_{\Gamma}
$$

от о.p. $u(x)$ задачи $(1.1),(1.2)$ или значением

$$
\left.\left[\frac{\partial u}{\partial \nu}+\left(\bar{\sigma}^{0}+\sum_{i=1}^{n} \bar{a}_{i} n_{i}\right) u\right]\right|_{\Gamma}
$$

от о.p. $u(x)$ задачи $(3.3),(3.4)$ будем понимать функционал из $W_{2}^{-1 / 2}(\Gamma)$, действующий на произвольную функцию $\psi \in W_{2}^{1 / 2}(\Gamma)$ по правилу

$$
\psi \rightarrow a[u, \overline{l \psi}]_{\mu}+\int_{\Omega} \alpha(x, D) u \cdot l \psi d x+\int_{\Gamma_{2}} \sigma_{2} u \cdot l \psi d s-\langle\widehat{f}, l \psi\rangle_{\Omega}
$$

в случае задачи (1.1), (1.2) или, соответственно,

$$
\psi \rightarrow a[u, \overline{l \psi}]_{\bar{\mu}}+\int_{\Omega} u \overline{\alpha(x, D) \overline{l \psi}} d x+\int_{\Gamma_{2}} u \overline{\sigma_{2} \overline{l \psi}} d s-\langle\widehat{f}, l \psi\rangle_{\Omega}
$$


в случае задачи $(3.3),(3.4)$, где $l \psi \in W_{2}^{1}(\Omega)$ - произвольное продолжение функции $\psi:\left.l \psi\right|_{\Gamma}=\psi$.

Нетрудно видеть, что правые части в (3.7а) и (3.7б) не зависят от выбора продолжения $l \psi$ и представляют собой функционалы из $W_{2}^{-1 / 2}(\Omega)$. Проверим это, например, для (3.7a). Пусть $l_{1} \psi$ и $l_{2} \psi$ - два продолжения функции $\psi$. Тогда $v=\overline{l_{2} \psi}-\overline{l_{1} \psi} \in \stackrel{\circ}{W} \frac{1}{2}(\Omega ; \Gamma)$, и следовательно, $v \in \stackrel{\circ}{W} \underset{2}{1}\left(\Omega ; \Gamma_{1}\right),\left.v\right|_{\Gamma_{2}}=0$. Поэтому разность значений правых частей $(3.7 \mathrm{a})$ при $l \psi$ равных, соответственно, $l_{2} \psi$ и $l_{1} \psi$ в силу $(3.5 \mathrm{a})$ равна

$$
\begin{aligned}
a[u, v]_{\mu} & +\int_{\Omega} \alpha(x, D) u \bar{v} d x+\int_{\Gamma_{2}} \sigma_{2} u \bar{v} d s-\langle\widehat{f}, \bar{v}\rangle_{\Omega} \\
& =a[u, v]_{\mu}+\int_{\Omega} \alpha(x, D) u \bar{v} d x+\int_{\Gamma_{2}} \sigma_{2} u \bar{v} d s-\langle f, \bar{v}\rangle_{\Omega}-\langle g, \bar{v}\rangle_{\Gamma_{2}}=0 .
\end{aligned}
$$

Элементарно проверяется при использовании в качестве $l \psi$ продолжения $l_{\Gamma}^{\Omega} \psi$, фигурируюшего в теореме 2.4 , и теоремы 2.3 вложения ограниченность функционала правой части $(3.7 \mathrm{a})$ в $W_{2}^{1 / 2}(\Gamma)$.

Далее, значение функционала правой части (3.7а) или (3.7б) на $\Gamma_{2}$ не зависит от выбора продолжения $\widehat{f}$ и равно заданному функционалу $g$. Действительно, если $\psi \in \stackrel{\circ}{W_{2}^{1 / 2}}\left(\Gamma_{2}\right)$, то $\left.\psi\right|_{\Gamma_{1}}=0, l \psi \in \stackrel{\circ}{W} \frac{1}{2}\left(\Omega ; \Gamma_{1}\right)$, а потому в силу соотношений $(3.5 \mathrm{a})$ и $(3.5 б)$ с $v=\overline{l \psi}$ и того, что $\widehat{f}$ совпадает с $f$ на $\stackrel{\circ}{W} \frac{1}{2}\left(\Omega ; \Gamma_{1}\right)$, получаем утверждаемое.

Однако на $\Gamma_{1}$ значения правых частей $(3.7 \mathrm{a})$ и $(3.7 б)$ существенно зависят от выбора продолжения $\widehat{f}$. В самом деле, пусть $\widehat{f}_{1} \in W_{2}^{-1}(\Omega)$ - некоторое продолжение функционала $f$, и пусть $\widehat{f}_{2}=\widehat{f}_{1}+\theta$, где $\theta \in W_{2}^{-1}(\Omega)$, действующий по формуле $\langle\theta, v\rangle_{\Omega}=\int_{\Gamma_{1}} v d s\left(\left.v\right|_{\Gamma}-\right.$ след $v$ на $\left.\Gamma\right)$. Тогда $\theta$ обращается в нуль на $\stackrel{\circ}{W} \underset{2}{1}\left(\Omega ; \Gamma_{1}\right)$, а потому $\widehat{f}_{2}$ - также продолжение функционала $f$. Следовательно, при одной и той же $\psi \in \stackrel{\circ}{W}{ }_{2}^{1 / 2}\left(\Gamma_{1}\right)$ разности правых частей как (3.7а), так и (3.7б), отвечающих продолжениям $\widehat{f}_{2}$ и $\widehat{f}_{1}$, отличаются друг от друга на отличный от нулевого функционал $\psi \rightarrow-\int_{\Gamma_{1}} \psi d s$.

Выясним на примере задачи Дирихле $-\Delta u+u=f$ в $\Omega,\left.u\right|_{\Gamma}=\varphi$, в случае, когда $\Omega$ - область с границей класса $C^{\infty}$, чему соответствует значение $\left.\frac{\partial u}{\partial \boldsymbol{n}}\right|_{\Gamma}$, отвечаюшее какому-либо продолжению $\widehat{f}$. В этом случае найдутся последовательности $\widehat{f}_{k}(x) \in C_{\mathbb{R}^{n}}^{\infty}(\bar{\Omega})$ и $\varphi_{k}(x) \in C^{\infty}(\Gamma)$ такие, что $\widehat{f}_{k} \rightarrow \widehat{f}_{\text {в }} W_{2}^{-1}(\Omega)$ и $\varphi_{k} \rightarrow \varphi$ в $W_{2}^{1 / 2}(\Gamma)$ при $k \rightarrow \infty$. Классические решения приближаюших задач Дирихле $-\Delta \widehat{u}_{k}+\widehat{u}_{k}=\widehat{f}_{k}$ в $\Omega,\left.\widehat{u}_{k}\right|_{\Gamma}=\varphi_{k}$, являются гладкими: $\widehat{u}_{k} \in C^{\infty}(\bar{\Omega})$, а потому для них определены в классическом смысле значения нормальных производных $\left.\frac{\partial \widehat{u}_{k}}{\partial \boldsymbol{n}}\right|_{\Gamma} \in C^{\infty}(\Gamma)$. Решения $\widehat{u}_{k}$ являются также и обобшенными, а потому в силу теоремы, следуюшей ниже, $\widehat{u}_{k}(x) \rightarrow u(x)$ в $W_{2}^{1}(\Omega)$ при $k \rightarrow \infty$, причем этот предел $u(x)$ зависит только от исходной правой части $f \in \stackrel{\circ}{W_{2}^{-1}}(\Omega ; \Gamma)$ и $\varphi$, но не 
от продолжений $\widehat{f}$. Но значения $\left.\frac{\partial \widehat{u}_{k}}{\partial \boldsymbol{n}}\right|_{\Gamma}$ стремятся в $W_{2}^{-1 / 2}(\Gamma)$ к значению $\left.\frac{\partial u}{\partial \boldsymbol{n}}\right|_{\Gamma}$ (зависяшему, как мы уже видели, от продолжения $\widehat{f}$ ).

В самом деле, исходя их формулы Грина, с помощью приближения $l \psi$ гладкими функциями получаем, что

$$
\int_{\Gamma} \frac{\partial \widehat{u}_{k}}{\partial \boldsymbol{n}} \psi d s=\int_{\Omega}\left[\left(\nabla \widehat{u}_{k}, \nabla l \psi\right)+\widehat{u}_{k} l \psi\right] d x-\int_{\Omega} \widehat{f}_{k} l \psi d x
$$

В силу определения $\left.\frac{\partial u}{\partial \boldsymbol{n}}\right|_{\Gamma}$, соответствуюшего продолжению $\widehat{f}$,

$$
\left\langle\frac{\partial u}{\partial \boldsymbol{n}}, \psi\right\rangle_{\Gamma}=\int_{\Omega}[(\nabla u, \nabla l \psi)+u l \psi] d x-\langle\widehat{f}, l \psi\rangle_{\Omega} .
$$

Поэтому, вычитая из предыдушего равенства последнее и производя очевидные оценки, получим оценку

$$
\left|\left\langle\left(\frac{\partial \widehat{u}_{k}}{\partial \boldsymbol{n}}-\frac{\partial u}{\partial \boldsymbol{n}}\right), \psi\right\rangle_{\Gamma}\right| \leqslant\left(\left\|\widehat{u}_{k}-u\right\|_{W_{2}^{1}(\Omega)}+\left\|\widehat{f}_{k}-\widehat{f}\right\|_{W_{2}^{-1}(\Omega)}\right)\|l \psi\|_{W_{2}^{1}(\Omega)} .
$$

Полагая в последнем неравенстве $l \psi=l_{\Gamma}^{\Omega} \psi$ - продолжению, фигурируюшему в теореме 2.4 , пользуясь оценкой (2.14) $\mathrm{c} l=1$, приходим к оценке

$\left\|\frac{\partial \widehat{u}_{k}}{\partial \boldsymbol{n}}-\frac{\partial u}{\partial \boldsymbol{n}}\right\|_{W_{2}^{-1 / 2}(\Gamma)} \leqslant c_{1}(\Omega, 1)\left(\left\|\widehat{u}_{k}-u\right\|_{W_{2}^{1}(\Omega)}+\left\|\widehat{f}_{k}-\widehat{f}\right\|_{W_{2}^{-1}(\Omega)}\right) \rightarrow 0$ при $k \rightarrow \infty$.

Заметим, что последнее утверждение может быть получено и непосредственно из теории, представленной в [6].

Отметим, кроме того, что в предыдушем примере, как это нетрудно проверить, при $\varphi \in C^{\infty}(\Gamma), f \in C^{\infty}(\bar{\Omega})$, значение $\left.\frac{\partial \widehat{u}}{\partial \boldsymbol{n}}\right|_{\Gamma}$, соответствуюшее "минимальному" продолжению $\widehat{f}$ в $W_{2}^{-1}(\Omega)$, а именно продолжению $f$ как функционала из $\stackrel{\circ}{W}_{2}^{-1}(\Omega ; \Gamma)$ с сохранением нормы в $W_{2}^{-1}(\Omega)$, равно вовсе не классическому значению $\left.\frac{\partial u}{\partial \boldsymbol{n}}\right|_{\Gamma}$, а значению $\left.\frac{\partial \widehat{u}}{\partial \boldsymbol{n}}\right|_{\Gamma}$ от решения задачи $-\Delta \widehat{u}+\widehat{u}=0$ в $\Omega,\left.\widehat{u}\right|_{\Gamma}=\varphi$.

Введем еще следующие понятия. Пусть, как и вьше, $\Gamma_{1}$ и $\Gamma_{2}-$ непересекаюшиеся открытые куски, составляюшие $\Gamma, m_{\Gamma}\left(\Gamma \backslash\left(\Gamma_{1} \cup \Gamma_{2}\right)\right)=0$. Обозначим через $W_{2, \mu}^{1 / 2}\left(\Gamma_{1} \rightarrow \Gamma\right)$ факторпространство $W_{2, \mu}^{1 / 2}(\Gamma) / \stackrel{\circ}{W_{2, \mu}^{1 / 2}}\left(\Gamma_{2}\right)$, т.е. подпространство функций $\varphi(x)$ из $W_{2, \mu}^{1 / 2}\left(\Gamma_{2}\right)$, допускающих продолжения $l \varphi \in W_{2, \mu}^{1 / 2}(\Gamma)$, с нормой

$$
\|\varphi\|_{W_{2, \mu}^{1 / 2}\left(\Gamma_{1} \rightarrow \Gamma\right)}=\inf _{\substack{\left.l \varphi \in W_{2, \mu}^{1 / 2}(\Gamma) \\ l \varphi\right|_{\Gamma_{1}}=\varphi}}\|l \varphi\|_{W_{2, \mu}^{1 / 2}(\Gamma)} .
$$

Через $W_{2, \mu}^{-1 / 2}\left(\Gamma_{1} \rightarrow \Gamma\right)$ обозначим пространство, сопряженное к $W_{2, \mu}^{1 / 2}\left(\Gamma_{1} \rightarrow \Gamma\right)$. Пространство $W_{2, \mu}^{1 / 2}\left(\Gamma_{1} \rightarrow \Gamma\right)$ (как факторпространство банаховых пространств) также является банаховым пространством (см., например, [31]). 
ОПрЕДЕЛЕНИЕ 3.3. Пусть функционал $h(x) \in W_{2, \mu}^{-1 / 2}(\Gamma)$ обрашается в нуль на $\Gamma_{2}:\langle h, \psi\rangle_{\Gamma}=0 \forall \psi \in \stackrel{\circ}{W_{2, \mu}^{1 / 2}}\left(\Gamma_{2}\right)$ (т.е. $\left.\operatorname{supp} h \subseteq \bar{\Gamma}_{1}\right)$. Под значением $h(x)$ на $\Gamma_{1}$ будем понимать линейный функционал $\left.h\right|_{\Gamma_{1}}$, действие которого на произвольную функцию $\varphi(x) \in W_{2, \mu}^{1 / 2}\left(\Gamma_{1} \rightarrow \Gamma\right)$ определяется по формуле

$$
\left\langle\left. h\right|_{\Gamma_{1}}, \varphi\right\rangle_{\Gamma_{1}}=\langle h, l \varphi\rangle_{\Gamma}
$$

где $l \varphi \in W_{2, \mu}^{1 / 2}(\Gamma)$ - произвольное продолжение функции $\varphi$.

Нетрудно видеть, что это определение не зависит от выбора продолжения $l \varphi$ функции $\varphi$, что $\left.h\right|_{\Gamma_{1}} \in W_{2, \mu}^{-1 / 2}\left(\Gamma_{1} \rightarrow \Gamma\right)$ и что $\left\|\left.h\right|_{\Gamma_{1}}\right\|_{W_{2, \mu}^{-1 / 2}\left(\Gamma_{1} \rightarrow \Gamma\right)} \leqslant\|h\|_{W_{2, \mu}^{-1 / 2}(\Gamma)}$.

В случае, когда $\Gamma_{1}$ - липшицев кусок $\Gamma$, пространство $W_{2, \mu}^{1 / 2}\left(\Gamma_{1} \rightarrow \Gamma\right)$ совпадает с пространством $W_{2, \mu}^{1 / 2}\left(\Gamma_{1}\right)$, и нормы этих двух пространств эквиваленты. В самом деле, включение $W_{2, \mu}^{1 / 2}\left(\Gamma_{1} \rightarrow \Gamma\right) \subseteq W_{2, \mu}^{1 / 2}\left(\Gamma_{1}\right)$ с оценкой

$$
\|\varphi\|_{W_{2, \mu}^{1 / 2}\left(\Gamma_{1}\right)} \leqslant\|\varphi\|_{W_{2, \mu}^{1 / 2}\left(\Gamma_{1} \rightarrow \Gamma\right)}
$$

элементарно следуют из (3.8). С другой стороны, если $\varphi \in W_{2, \mu}^{1 / 2}\left(\Gamma_{1}\right)$, то по теореме 2.1.2) сушествует продолжение $l \varphi \in W_{2, \mu}^{1 / 2}(\Gamma)$ функции $\varphi$ такое, что выполняется оценка $(2.12)$ с $\Lambda=\Gamma_{1}$ и $l=1 / 2$, а потому $\|\varphi\|_{W_{2, \mu}^{1 / 2}\left(\Gamma_{1} \rightarrow \Gamma\right)} \leqslant c\left(\Gamma_{1}, \Gamma\right)\|\varphi\|_{W_{2, \mu}^{1 / 2}\left(\Gamma_{1}\right)}$. Естественно, что в этом случае и пространство $W_{2, \mu}^{-1 / 2}\left(\Gamma_{1} \rightarrow \Gamma\right)$ совпадает с пространством $W_{2, \mu}^{-1 / 2}\left(\Gamma_{1}\right)$.

Перейдем к основной теореме этого параграфа.

ТЕОРЕМА 3.1. При любом комплексном $\mu$ размерность пространства обобщенных решений однородной задачи (1.1), (1.2) конечна и совпадает с размерностью пространства о.р. однородной сопряжснной задачи (3.3), (3.4). $\Pi p u \quad f(x) \in \stackrel{\circ}{W}_{2}^{-1}\left(\Omega ; \Gamma_{1}\right), g(x) \in \stackrel{\circ}{W}_{2, \mu}^{-1 / 2}\left(\Gamma_{2}\right), \varphi(x) \in W_{2}^{1 / 2}\left(\Gamma_{1} \rightarrow \Gamma\right)^{1)}$ задача (1.1), (1.2) имеет о.р. тогда и только тогда, когда

$$
\left\langle f, \bar{z}^{*}\right\rangle_{\Omega}-\left\langle\frac{\partial \bar{z}^{*}}{\partial \nu}, \varphi\right\rangle_{\Gamma_{1}}+\left\langle g, \bar{z}^{*}\right\rangle_{\Gamma_{2}}=0
$$

для любого о.р. $z^{*}$ однородной сопряженной задачи (3.3), (3.4), а неоднородная задача (3.3), (3.4) имеет о.р. тогда и только тогда, когда

$$
\langle f, \bar{z}\rangle_{\Omega}-\left\langle\frac{\partial \bar{z}}{\partial \nu}, \varphi\right\rangle_{\Gamma_{1}}+\langle g, \bar{z}\rangle_{\Gamma_{2}}=0
$$

\footnotetext{
1) Последнее условие в силу теоремы 2.3 является необходимым условием существования обобщенного решения в $W_{2}^{1}(\Omega)$.
} 
для любого о.р. z однородной задачи (1.1), (1.2). Существует такое $\mu_{*}>0$, что при $\operatorname{Re} \mu \geqslant \mu_{*}$ каждая из задач (1.1), (1.2) и (3.3), (3.4) однозначно разрешима и для их решений $и(x)$ имеют место оценки

$$
\begin{aligned}
\|u\|_{W_{2,|\mu|}^{1}(\Omega)} \leqslant & C(1+|\operatorname{Im} \mu| / \operatorname{Re} \mu) \\
& \times\left(\|f\|_{W_{2,|\mu|}^{-1}\left(\Omega ; \Gamma_{1}\right)}+c_{1}\|\varphi\|_{W_{2,|\mu|}^{1 / 2}\left(\Gamma_{1} \rightarrow \Gamma\right)}+c_{2}\|g\|_{W_{2,|\mu|}^{-1 / 2}\left(\Gamma_{2}\right)}\right),
\end{aligned}
$$

әде $C, c_{1}$ и с ${ }_{2}$-некоторые постоянные, не зависящие от $\mu$. В (3.10)

$$
\begin{gathered}
\left\langle\frac{\partial \bar{z}}{\partial \nu}, \varphi\right\rangle_{\Gamma_{1}}=\overline{\left\langle\frac{\partial z}{\partial \nu}, \bar{\varphi}\right\rangle_{\Gamma_{1}}}, \quad\left\langle\frac{\partial \bar{z}^{*}}{\partial \nu}, \varphi\right\rangle_{\Gamma_{1}}=\overline{\left\langle\frac{\partial z^{*}}{\partial \nu}, \bar{\varphi}\right\rangle_{\Gamma_{1}}}, \\
\left\langle\frac{\partial z}{\partial \nu}, \varphi\right\rangle_{\Gamma_{1}}=\left\langle\left.\left[\frac{\partial z}{\partial \nu}+\sigma^{0} z\right]\right|_{\Gamma_{1}}, \varphi\right\rangle_{\Gamma_{1}}, \\
\left\langle\frac{\partial z^{*}}{\partial \nu}, \varphi\right\rangle_{\Gamma_{1}}=\left\langle\left.\left[\frac{\partial z^{*}}{\partial \nu}+\left(\bar{\sigma}^{0}+\sum_{i=1}^{n} \bar{a}_{i} n_{i}\right) z^{*}\right]\right|_{\Gamma_{1}}, \varphi\right\rangle_{\Gamma_{1}}
\end{gathered}
$$

(см. определения 3.2 и 3.3 ; при определении функционалов

$$
\left.\left[\frac{\partial z}{\partial \nu}+\sigma^{0} z\right]\right|_{\Gamma}
$$

$u$

$$
\left.\left[\frac{\partial z^{*}}{\partial \nu}+\left(\bar{\sigma}^{0}+\sum_{i=1}^{n} \bar{a}_{i} n_{i}\right) z^{*}\right]\right|_{\Gamma}
$$

по формулам (3.7а) и (3.7б) продолжения $\widehat{f}$ полагаются нулевьци).

$B$ случае, когда $\Gamma_{1}$ - липшичев кусок, всюду в вышеприведенной формулировке теоремь $W_{2,|\mu|}^{1 / 2}\left(\Gamma_{1} \rightarrow \Gamma\right)$ следует заменить на все пространство $W_{2,|\mu|}^{1 / 2}\left(\Gamma_{1}\right)$.

Отметим, что оценка (3.11) для случая однородной задачи Дирихле или 3-ей краевой задачи представляет собой хорошо известную оценку резольвенты эллиптического оператора вне некоторой параболы, содержащей внутри себя отрицательную полуось на плоскости $\lambda=\mu^{2}$.

ДОКАЗАТЕЛЬСТво ТЕОРЕМЫ 3.1 проведем в четыре этапа.

І-й этап. Задачи (1.1), (1.2) и (3.3), (3.4) с $a_{i}(x)=0, i=1, \ldots, n, a_{0}^{2}(x)=0$, $\sigma_{2}(x)=0, \mu>0, \varphi(x)=0$.

При таких предположениях задача (3.3), (3.4) совпадает с задачей (1.1), (1.2). Форма $a[u, v]_{\mu}$, определенная для $u(x), v(x) \in \stackrel{\circ}{W} \underset{2}{1}, \mu\left(\Omega ; \Gamma_{1}\right)$ выражением (3.2а), эрмитова и при достаточно больших $\mu: \mu \geqslant \mu_{0}>0$ эквивалентна скалярному произведению (2.6). В самом деле, в силу (1.5), (1.6) и существенной ограниченности $a_{i j}(x)$ и $q(x)$, имеем при $\mu>0$

$$
\begin{aligned}
& 2 c^{2}\|u\|_{W_{2, \mu}^{1}(\Omega)}^{2}+\underset{x \in \Omega}{\operatorname{essinf}} a_{0}^{1}(x) \cdot\|u\|_{L_{2}(\Omega)}^{2}+\underset{x \in \Gamma_{2}}{\operatorname{ess} \inf } \sigma_{1}(x) \cdot\|u\|_{L_{2}\left(\Gamma_{2}\right)}^{2} \\
& \leqslant a[u, u]_{\mu} \leqslant \frac{1}{2} C^{2}\|u\|_{W_{2, \mu}^{1}(\Omega)}^{2}+\underset{x \in \Omega}{\operatorname{ess} \sup } a_{0}^{1}(x) \cdot\|u\|_{L_{2}(\Omega)}^{2}+\underset{x \in \Gamma_{2}}{\operatorname{ess~sup} \sigma_{1}(x) \cdot\|u\|_{L_{2}\left(\Gamma_{2}\right)}^{2} .}
\end{aligned}
$$


Используя выражение (2.6), неравенство вложения (2.13) и выражение (2.7), получим оценки

$$
\begin{aligned}
& \mu^{2}\|u\|_{L_{2}(\Omega)}^{2} \leqslant\|u\|_{W_{2, \mu}^{1}(\Omega)}^{2}, \\
& \mu\|u\|_{L_{2}\left(\Gamma_{2}\right)}^{2} \leqslant c_{0}^{2}(\Omega, 1)\|u\|_{W_{2, \mu}^{1}(\Omega)}^{2} .
\end{aligned}
$$

Отсюда следует существование такого $\mu_{0}>0$, что при $\mu \geqslant \mu_{0}$

$$
c^{2}\|u\|_{W_{2, \mu}^{1}(\Omega)}^{2} \leqslant a[u, u]_{\mu} \leqslant C^{2}\|u\|_{W_{2, \mu}^{1}(\Omega)}^{2}
$$

где постоянные $c>0, C>0$ не зависят от $\mu$, причем, если $\sigma_{1}(x) \geqslant 0$ п.в. на $\Gamma_{2}$, то $\mu_{0}>0$ можно взять произвольно малым ( $C$ в этом случае будет зависеть от $\left.\mu_{0}\right)$.

В рассматриваемом сейчас случае функция $u(x) \in \stackrel{\circ}{W_{2, \mu}^{1}}\left(\Omega ; \Gamma_{1}\right)$ является о.р. задачи $(1.1),(1.2)$, если $\forall v(x) \in \stackrel{\circ}{W} \underset{2, \mu}{1}\left(\Omega ; \Gamma_{1}\right)$

$$
a[u, v]_{\mu}=\langle F, \bar{v}\rangle_{\Omega}
$$

где

$$
\langle F, \bar{v}\rangle_{\Omega}=\langle f, \bar{v}\rangle_{\Omega}+\langle g, \bar{v}\rangle_{\Gamma_{2}}
$$

Функционал $\overline{\langle F, \bar{v}}_{\Omega}$ представляет собой элемент из $\stackrel{\circ}{W} \underset{2, \mu}{-1}\left(\Omega ; \Gamma_{1}\right)$, поскольку с использованием (2.13)

$$
\left|\langle F, \bar{v}\rangle_{\Omega}\right| \leqslant\left\{\|f\|_{\stackrel{\circ}{W}_{2, \mu}^{-1}\left(\Omega, \Gamma_{1}\right)}+c_{0}(\Omega, 1)\|g\|_{\stackrel{\circ}{2, \mu}_{2,1}^{-1 / 2}\left(\Gamma_{2}\right)}\right\}\|v\|_{W_{2, \mu}^{1}(\Omega)},
$$

и норма этого функционала не превосходит выражения в фигурных скобках. Используя в качестве скалярного произведения в $\stackrel{\circ}{W} \underset{2, \mu}{1}\left(\Omega ; \Gamma_{1}\right)$ форму $a[u, v]_{\mu}$, на основании теоремы Рисса заключаем, что существует $u(x) \in \stackrel{\circ}{W} \underset{2}{1}, \mu\left(\Omega ; \Gamma_{1}\right)$ такая, что $a[v, u]_{\mu}={\overline{\langle F, \bar{v}\rangle_{\Omega}}}_{\Omega}$, т.е. $(3.13)$ выполняется $\forall v \in \stackrel{\circ}{W} \underset{2, \mu}{1}\left(\Omega ; \Gamma_{1}\right)$ и при этом значение $a[u, u]_{\mu}^{1 / 2}$ равно норме функционала $F$ при выбранной нормировке $W_{2, \mu}^{1}(\Omega)$. Поэтому (3.11) имеет место для $\mu \geqslant \mu_{0}$.

Для дальнейшего вводим линейный оператор $G_{\mu}: \stackrel{\circ}{\underset{W}{2}} \underset{2, \mu}{-1}\left(\Omega ; \Gamma_{1}\right) \rightarrow \stackrel{\circ}{W_{2, \mu}^{1}}\left(\Omega ; \Gamma_{1}\right)$, действующий на произвольный $F \in \stackrel{\circ}{W_{2}^{-1}}\left(\Omega ; \Gamma_{1}\right)$ по форомуле $G_{\mu} F=u$, где $u-$ решение задачи (3.13). Таким образом,

$$
a\left[G_{\mu} f, v\right]_{\mu}=\langle f, \bar{v}\rangle_{\Omega}, \quad f \in \stackrel{\circ}{W}_{2, \mu}^{-1}\left(\Omega ; \Gamma_{1}\right), \quad \forall v \in \stackrel{\circ}{W}_{2, \mu}^{1}\left(\Omega ; \Gamma_{1}\right) .
$$

Свойства оператора $G_{\mu}$ при $\mu \geqslant \mu_{0}$. 
$1^{\circ}$. Оператор $G_{\mu}$ представляет собой гомеоморфизм пространства $\stackrel{\circ}{W}_{2, \mu}^{-1}\left(\Omega ; \Gamma_{1}\right)$ на пространство $\stackrel{\circ}{W} \frac{1}{2, \mu}\left(\Omega ; \Gamma_{1}\right)$, причем норми $G_{\mu}$ и $G_{\mu}^{-1}$ могут быть оценены постоянными, не зависящими от $\mu$.

В самом деле, оператор $G_{\mu}$ действует взаимно однозначно: из (3.16) имеем, что если $G_{\mu} f=0$, то и $f$ как функционал равен нулю. Далее, $G_{\mu}$ отображает на все $\stackrel{\circ}{W}{ }_{2, \mu}^{1}\left(\Omega ; \Gamma_{1}\right)$. Действительно, $\forall u \in \stackrel{\circ}{W_{2, \mu}^{1}}\left(\Omega ; \Gamma_{1}\right)$ функционал $v \rightarrow a[u, \bar{v}]_{\mu}$ представляет собой в силу оценки

$$
\left|a[u, v]_{\mu}\right| \leqslant C^{2}\|u\|_{W_{2, \mu}^{1}(\Omega)}\|v\|_{W_{2, \mu}^{1}(\Omega)},
$$

устанавливаемой аналогично (3.12), некоторьй элемент $f \in \stackrel{\circ}{W_{2, \mu}^{-1}}\left(\Omega ; \Gamma_{1}\right)$. Следовательно, $G_{\mu} f=u$ и

$$
\|f\|_{\stackrel{\circ}{W}_{2, \mu}^{-1}\left(\Omega ; \Gamma_{1}\right)}=\left\|G_{\mu}^{-1} u\right\|_{\stackrel{\circ}{W}_{2, \mu}^{-1}\left(\Omega ; \Gamma_{1}\right)} \leqslant C^{2}\|u\|_{\stackrel{\circ}{W}_{2, \mu}^{1}\left(\Omega ; \Gamma_{1}\right)} \quad \forall u \in \stackrel{\circ}{W_{2, \mu}^{1}}\left(\Omega, \Gamma_{1}\right) .
$$

$2^{\circ}$. Сужение оператора $G_{\mu}$ на $L_{2}(\Omega)$, рассматриваемое как оператор в $L_{2}(\Omega)$, представляет собой вполне непрерывный самосопряженный неотрицательный оператор, у которого 0 не является собственным значением.

В самом деле, поскольку

$$
\stackrel{\circ}{W}_{2, \mu}^{-1}\left(\Omega ; \Gamma_{1}\right) \supset L_{2}(\Omega) \supset \stackrel{\circ}{W} \underset{2, \mu}{1}\left(\Omega ; \Gamma_{1}\right)
$$

оператор $G_{\mu}$ определен на всем $L_{2}(\Omega)$ и действует в $L_{2}(\Omega)$. Далее, полагая $\forall f_{1}, f_{2} \in L_{2}(\Omega)$ в $(3.16) f=f_{1}, v=G_{\mu} f_{2}$, имеем

$$
\begin{aligned}
\left(f_{1}, G_{\mu} f_{2}\right)_{L_{2}(\Omega)} & =\left\langle f_{2}, \overline{G_{\mu} f_{2}}\right\rangle_{\Omega}=a\left[G_{\mu} f_{1}, G_{\mu} f_{2}\right]_{\mu} \\
& =\overline{a\left[G_{\mu} f_{2}, G_{\mu} f_{1}\right]_{\mu}}=\overline{\left\langle f_{2}, \overline{G_{\mu} f_{1}}\right\rangle_{\Omega}}=\left(G_{\mu} f_{1}, f_{2}\right)_{L_{2}(\Omega)}
\end{aligned}
$$

Отсюда, в частности,

$$
\left(G_{\mu} f, f\right)_{L_{2}(\Omega)}=a\left[G_{\mu} f, G_{\mu} f\right]_{\mu} \geqslant 0 \quad \forall f \in L_{2}(\Omega) .
$$

Далее, если $G_{\mu} f=0$ для некоторой $f \in L_{2}(\Omega)$, то в силу предыдушего свойства $1^{\circ} f=0$ как элемент пространства $\stackrel{\circ}{W} \underset{2, \mu}{-1}\left(\Omega ; \Gamma_{1}\right)$, что означает, что

$$
\int_{\Omega} f v d x=0 \quad \forall v \in \stackrel{\circ}{W_{2, \mu}^{1}}\left(\Omega ; \Gamma_{1}\right) .
$$

Но тогда согласно обобшенной лемме Дюбуа-Реймона $f(x)=0$ п.в. на $\Omega$. Компактность оператора $G_{\mu}$ как оператора в $L_{2}(\Omega)$ следует из того, что этот оператор представляет собой композицию оператора $G_{\mu}$ и оператора вложения пространства $\stackrel{\circ}{W} \frac{1}{2, \mu}\left(\Omega ; \Gamma_{1}\right)$ в $L_{2}(\Omega)$, а последний оператор вследствие теоремы 2.5 вполне непрерывен. 
$3^{\circ}$. В силу свойства $2^{\circ}$ оператора $G_{\mu}$ существует компактный положительный оператор $G_{\mu}^{1 / 2}$ (см., например, [30]). Оператор $G_{\mu}^{1 / 2}$ на самом деле действует ограниченно из $L_{2}(\Omega)$ на все $\stackrel{\circ}{W} \underset{2, \mu}{1}\left(\Omega ; \Gamma_{1}\right)$.

Установим это. Используя (3.17), получим $\forall f_{1}, f_{2} \in L_{2}(\Omega)$ равенство

$$
a\left[G_{\mu} f_{1}, G_{\mu} f_{2}\right]_{\mu}=\left(G_{\mu}^{1 / 2} f_{1}, G_{\mu}^{1 / 2} f_{2}\right)_{L_{2}(\Omega)} .
$$

Вьполняя замены $v_{i}=G_{\mu}^{1 / 2} f_{i}, i=1,2$, находим, что

$$
a\left[G_{\mu}^{1 / 2} v_{1}, G_{\mu}^{1 / 2} v_{2}\right]_{\mu}=\left(v_{1}, v_{2}\right)_{L_{2}(\Omega)}
$$

для всех таких $v_{1}$ и $v_{2}$. Но область значений оператора $G_{\mu}^{1 / 2}$, определенного на $L_{2}(\Omega)$, плотна в $L_{2}(\Omega)$. В самом деле, из условия

$$
\left(G_{\mu}^{1 / 2} f, g\right)_{L_{2}(\Omega)}=0 \quad \forall f \in L_{2}(\Omega)
$$

следует равенство $\left(f, G_{\mu}^{1 / 2} g\right)_{L_{2}(\Omega)}=0$, откуда $G_{\mu}^{1 / 2} g=0$, и в силу положительности $G_{\mu}^{1 / 2}$ имеем, что $g=0$.

Таким образом, производя замыкание в (3.18) с таких $v_{i}=G_{\mu}^{1 / 2} f_{i}$ до любых $v_{i} \in L_{2}(\Omega), i=1,2$, получаем справедливость $3^{\circ}$ и формулы $(3.18) \forall v_{i} \in L_{2}(\Omega)$, $i=1,2$.

II-й этап. Задачи (1.1), (1.2) и (3.3), (3.4) при общих сфформулированных в теореме предположениях с дополнительными условиями: $\mu>\mu_{0}>0, \varphi(x)=0$ в (1.2) и (3.4) ( $\mu_{0}$ - то же, что и на I-м этапе).

Сведем задачи (3.5а) и (3.5б) к уравнениям Фредгольма в пространстве $\stackrel{\circ}{W}_{2, \mu}^{1}\left(\Omega ; \Gamma_{1}\right)$. Введем билинейную формулу

$$
\begin{gathered}
B(u, v)=B_{1}(u, v)+B_{2}(u, v) \\
B_{1}(u, v)=\int_{\Omega} \alpha(x, D) u v d x, \quad B_{2}(u, v)=\int_{\Gamma_{2}} \sigma_{2} u v d x
\end{gathered}
$$

$\forall u, v \in \stackrel{\circ}{W} \frac{1}{2, \mu}\left(\Omega ; \Gamma_{1}\right)$. Эта форма непрерывна на $\stackrel{\circ}{W} \underset{2, \mu}{1}\left(\Omega ; \Gamma_{1}\right) \times \stackrel{\circ}{W} \frac{1}{2, \mu}\left(\Omega ; \Gamma_{1}\right)$, поскольку с использованием неравенства Коши-Буняковского, неравенства вложения (2.13) и определений (2.6) и (2.9) получаем оценку

$$
|B(u, v)| \leqslant \mu^{-1} C\|u\|_{W_{2, \mu}^{1}(\Omega)}\|v\|_{W_{2, \mu}^{1}(\Omega)},
$$

где постоянная $C$ не зависит от $\mu$. Отсюда следует, что форма $B(u, v)$ представляет собой при фиксированной $u \in \stackrel{\circ}{\stackrel{1}{W}} \underset{2, \mu}{1}\left(\Omega ; \Gamma_{1}\right)$ функционал $B(u, \cdot) \in \stackrel{\circ}{W} \underset{2, \mu}{-1}\left(\Omega ; \Gamma_{1}\right)$ по переменной $v$ (с нормой, не превосходящей $\left.C \mu^{-1}\|u\|_{W_{2, \mu}^{1}(\Omega)}\right)$ и точно также $\bar{B}(u, v)=\overline{B(\bar{u}, \bar{v})}$ как функционал $\bar{B}(\cdot, v)$ по первой переменной $u$ при фиксированной $v$ есть элемент $\stackrel{\circ}{W} \underset{2, \mu}{-1}\left(\Omega ; \Gamma_{1}\right)$.

Поэтому линейные операторы $K_{\mu}$ и $K_{\mu}^{\prime}$, определяемые по формулам $K_{\mu} u=$ $G_{\mu} B(u, \cdot)$ и $K_{\mu}^{\prime} u=G_{\mu} \bar{B}(\cdot, u)$, а именно, соотношениями

$$
a\left[K_{\mu} u, v\right]_{\mu}=B(u, \bar{v}), \quad a\left[K_{\mu}^{\prime} u, v\right]_{\mu}=\overline{B(v, \bar{u})}
$$

$\forall v \in \stackrel{\circ}{W}{ }_{2, \mu}^{1}\left(\Omega ; \Gamma_{1}\right)$, представляют собой непрерывные операторы в $\stackrel{\circ}{W} \underset{2, \mu}{1}\left(\Omega ; \Gamma_{1}\right)$ (норма каждого не превосходит $C / \mu$ ). Эти операторы обладают следующими свойствами. 
$1^{\circ} . K_{\mu}$ и $K_{\mu}^{\prime}-$ вполне непрерьвнье операторьи на $\stackrel{\circ}{\mathrm{W}} \underset{2, \mu}{1}\left(\Omega ; \Gamma_{1}\right)$.

Это достаточно установить для $K_{\mu}$. Используя разложение (3.19), можем представить

$$
K_{\mu}=K_{\mu, 1}+K_{\mu, 2}
$$

где

$$
K_{\mu, 1} u=G_{\mu} B_{1}(u, \cdot)=G_{\mu} \alpha(x, D) u, \quad K_{\mu, 2} u=G_{\mu} B_{2}(u, \cdot) .
$$

Представим $K_{\mu, 1}=G_{\mu}^{1 / 2} G_{\mu}^{1 / 2} \alpha(x, D) u$. В силу свойства $3^{\circ}$ оператора $G_{\mu}$ оператор $G_{\mu}^{1 / 2} \alpha(x, D)$ действует ограниченно в пространстве $\stackrel{\circ}{W} \underset{2, \mu}{1}\left(\Omega ; \Gamma_{1}\right)$, а потому вполне непрерывно из $\stackrel{\circ}{W} \underset{2, \mu}{1}\left(\Omega ; \Gamma_{1}\right)$ в $L_{2}(\Omega)$. Поэтому $K_{\mu, 1}$ является вполне непрерывным оператором в $\stackrel{\circ}{W} \underset{2}{1}, \mu\left(\Omega ; \Gamma_{1}\right)$.

Перейдем к оператору $K_{\mu, 2}$. Для формы $B_{2}(u, v)$ имеем с использованием неравенства вложения (2.13) оценку

$$
\left|B_{2}(u, v)\right| \leqslant C\|u\|_{L_{2}\left(\Gamma_{2}\right)}\|v\|_{L_{2}\left(\Gamma_{2}\right)} \leqslant \mu^{-1 / 2} C\|u\|_{L_{2}\left(\Gamma_{2}\right)}\|v\|_{W_{2, \mu}^{1}(\Omega)}
$$

(отсюда норма функционала $v \rightarrow B_{2}(u, v)$ не превосходит $\left.C \mu^{-1 / 2}\|u\|_{L_{2}\left(\Gamma_{2}\right)}\right)$. Пусть теперь $u_{j}$ - ограниченная последовательность в $\stackrel{\circ}{W} \underset{2, \mu}{1}\left(\Omega ; \Gamma_{1}\right)$. Тогда в силу теоремы 2.5 последовательность $\left.u_{j}\right|_{\Gamma_{2}}$ компактна в $L_{2}\left(\Gamma_{2}\right)$ и из нее можно выделить сходящуюся в $L_{2}\left(\Gamma_{2}\right)$ подпоследовательность $\left.u_{j_{\nu}}\right|_{\Gamma_{2}}$. Поэтому подпоследовательность $K_{\mu, 2} u_{j_{\nu}}=G_{\mu} B_{2}\left(u_{j_{\nu}}, \cdot\right)$ будет фундаментальна в $\stackrel{\circ}{W} \underset{2, \mu}{1}\left(\Omega ; \Gamma_{1}\right)$. Итак, оператор $K_{\mu, 2}$ переводит всякую ограниченную последовательность в компактную, а потому является также вполне непрерывным в $\stackrel{0}{W} \underset{2, \mu}{1}\left(\Omega ; \Gamma_{1}\right)$.

$2^{\circ} . K_{\mu}^{\prime}$ сопряжен оператору $K_{\mu}$ относительно скалярного произведения $a[u, v]_{\mu}$.

В самом деле, заменяя во втором соотношении (3.20) $u$ на $v$, а $v$ на $u$, получим

$$
a\left[u, K_{\mu}^{\prime} v\right]_{\mu}=B(u, \bar{v})=a\left[K_{\mu} u, v\right]_{\mu} \quad \forall u, v \in \stackrel{\circ}{W}_{2, \mu}^{1}\left(\Omega ; \Gamma_{1}\right)
$$

Воспользуемся теперь в соотношениях (3.5а) и (3.5б) соотношениями (3.20) и (3.16), последним с $f$, замененным на $F$, определяемьм (3.14). В результате (3.5а) и (3.5б) можем переписать, соответственно, в виде

$$
a\left[\left(u+K_{\mu} u-G_{\mu} F\right), v\right]_{\mu}=0 \quad \text { и } a\left[\left(u+K_{\mu}^{\prime} u-G_{\mu} F\right), v\right]_{\mu}=0 .
$$

В силу произвольности $v$ отсюда находим, что соотношения (3.5а) и (3.5б) эквивалентны, соответственно, уравнениям $\Phi$ редгольма второго рода

$$
\begin{aligned}
u+K_{\mu} u & =G_{\mu} F \\
u+K_{\mu}^{\prime} u & =G_{\mu} F
\end{aligned}
$$


в пространстве $\stackrel{\circ}{W} \underset{2, \mu}{1}\left(\Omega ; \Gamma_{1}\right)$.

В силу хорошо известной абстрактной теории таких уравнений (см., например, [30], а также [18, гл. II]), размерность пространства решений $z$ однородного уравнения (3.21a) конечна и совпадает с размерностью пространства решений $z^{*}$ однородного уравнения (3.21б). Уравнения (3.21a) или (3.21б) разрешимы тогда и только тогда, когда, соответственно,

$$
a\left[G_{\mu} F, z^{*}\right]_{\mu}=0 \quad \forall z^{*} \quad \text { или } \quad a\left[G_{\mu} F, z\right]_{\mu}=0 \quad \forall z .
$$

Но в силу предыдушего $z$ и $z^{*}$ являются обобщенньпи решениями задач (1.1), (1.2) и $(3.3),(3.4)$, соответственно. Кроме того, в силу (3.16) и (3.14) необходимые и достаточные условия (3.22) сушествования обобшенных решений задач $(1.1),(1.2)$ и $(3.3),(3.4)$ с $\varphi(x)=0$ приводятся, соответственно, к виду (3.10а) и (3.10б).

III-й этап. $\mu$-произвольное, $\varphi(x)=0$ в (1.2) и (3.4).

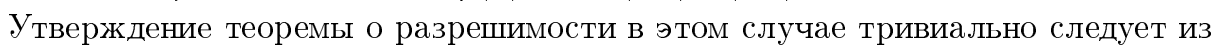
уже установленных на II-ом этапе утверждений. В самом деле, уравнение (1.1) всегда можно переписать в виде $\widetilde{L}(x, D, \widetilde{\mu})=f$ с $\widetilde{a}_{i j}(x)=a_{i j}(x), \widetilde{a}_{i}(x)=a_{i}(x)$, $\widetilde{a}_{0}(x)=a_{0}(x)+\left(\mu^{2}-\widetilde{\mu}^{2}\right) q(x)$, и с $\widetilde{\mu}>\mu_{0}$ - достаточно большим неотрицательным.

Перейдем к выводу оценки (3.11). Обозначим $\mu_{1}=\operatorname{Re} \mu, \mu_{2}=\operatorname{Im} \mu$. Пусть, например, $u(x)$ - o.p. задачи (1.1), (1.2) с $\varphi(x)=0$. Положим $\sigma_{1}(x)=\operatorname{Re} \sigma(x)$, $\sigma_{2}(x)=i \operatorname{Im} \sigma(x)$. Далее положим в соотношении (3.5a) $v=u$ и отделим в нем действительные и мнимые части. Приходим к двум равенствам

$$
\begin{gathered}
a[u, u]_{|\mu|}-2 \mu_{2}^{2} \int_{\Omega} q|u|^{2} d x=\operatorname{Re}\langle F, \bar{u}\rangle_{\Omega}-\operatorname{Re} \int_{\Omega} \alpha(x, D) u \bar{u} d x, \\
2 \mu_{1} \mu_{2} \int_{\Omega} q|u|^{2} d x=\operatorname{Im}\langle F, \bar{u}\rangle_{\Omega}-\operatorname{Im} \int_{\Omega} \alpha(x, D) u \bar{u} d x-\int_{\Gamma_{2}} \operatorname{Im} \sigma \cdot|u|^{2} d s,
\end{gathered}
$$

где функционал $F$ определен (3.14). Исключая из этих двух соотношений

$$
\int_{\Omega} q|u|^{2} d x
$$

получаем равенство

$$
\begin{aligned}
a[u, u]_{|\mu|}=\operatorname{Re}\langle F, \bar{u}\rangle_{\Omega}+\frac{\mu_{2}}{\mu_{1}} & \operatorname{Im}\langle F, \bar{u}\rangle_{\Omega}-\operatorname{Re} \int_{\Omega} \alpha(x, D) u \bar{u} d x \\
& -\frac{\mu_{2}}{\mu_{1}} \operatorname{Im} \int_{\Omega} \alpha(x, D) u \bar{u} d x-\frac{\mu_{2}}{\mu_{1}} \int_{\Gamma_{2}} \operatorname{Im} \sigma \cdot|u|^{2} d s .
\end{aligned}
$$

Оценивая сверху правую часть этого равенства с использованием неравенств

$$
\left|\int_{\Omega} \alpha(x, D) u \bar{u} d x\right|,\left.\quad\left|\int_{\Gamma_{2}} \operatorname{Im} \sigma \cdot\right| u\right|^{2} d s \mid \leqslant \frac{C}{|\mu|}\|u\|_{W_{2,|\mu|}^{1}(\Omega)}^{2}
$$


(получаемых точно так же, как и приведенная выше оценка для формы $B(u, v)$ ) и левой оценки (3.12), получим, что для $\mu_{1}>\mu_{0}$

$$
\begin{aligned}
\|u\|_{W_{2,|\mu|}^{1}(\Omega)}^{2} \leqslant C^{2}\left(1+\left|\mu_{2}\right| / \mu_{1}\right) \| F & \cdot\|u\|_{W_{2,|\mu|}^{1}(\Omega)} \\
& +C_{1}\left(1+\left|\mu_{2}\right| / \mu_{1}\right)|\mu|^{-1}\|u\|_{W_{2,|\mu|}^{1}(\Omega)},
\end{aligned}
$$

где $\|F\|$ - норма функционала $F$, постоянные $C$ и $C_{1}$ не зависят от $\mu, \mu_{1}>\mu_{0}$. Поскольку $\left(1+\left|\mu_{2}\right| / \mu_{1}\right)|\mu|^{-1} \leqslant 2 \mu_{1}^{-1}$, то, если еще потребовать, чтобы выполнялось $\mu_{1}=\operatorname{Re} \mu \geqslant 4 C_{1}$, т.е. если взять $\mu_{*} \geqslant \max \left\{\mu_{0}, 4 C_{1}\right\}$, при $\mu_{1} \geqslant \mu_{*}$ выполняется оценка $C_{1}\left(1+\left|\mu_{2}\right| / \mu_{1}\right)|\mu|^{-1} \leqslant 1 / 2$. При этом (3.23) с вытекающей из $(3.15)$ оценки для $\|F\|$ дают оценку (3.11).

Для обобшенного решения $u(x)$ задачи $(3.3),(3.4)$ совершенно аналогично получается такая же оценка (3.11).

IV-й этап. Общий случай, $\varphi \not \equiv 0$ в (1.2) или в (3.4).

Сначала рассмотрим случай $\operatorname{Re} \mu \geqslant \mu_{*}>0$. Пусть $\varphi(x) \in W_{2,|\mu|}^{1 / 2}\left(\Gamma_{1} \rightarrow \Gamma\right)$. В силу (3.8) найдется продолжение $l \varphi \in W_{2,|\mu|}^{1 / 2}(\Gamma)$ функции $\varphi$ такое, что

$$
\|l \varphi\|_{W_{2,|\mu|}^{1 / 2}(\Gamma)} \leqslant 2\|\varphi\|_{W_{2,|\mu|}^{1 / 2}\left(\Gamma_{1} \rightarrow \Gamma\right)} .
$$

Далее, функция $u_{0}(x)=l_{\Gamma}^{\Omega} \cdot l \varphi$, где $l_{\Gamma}^{\Omega}-$ оператор продолжения, фигурирующий в теореме 2.4 , принадлежит $W_{2,|\mu|}^{1}(\Omega),\left.u_{0}(x)\right|_{\Gamma_{1}}=\varphi(x)$ и

$$
\left\|u_{0}\right\|_{W_{2,|\mu|}^{1}(\Omega)} \leqslant C\|\varphi\|_{W_{2,|\mu|}^{1 / 2}\left(\Gamma_{1} \rightarrow \Gamma\right)},
$$

где $C$ - некоторая постоянная, не зависящая от $|\mu|$. Если искать решение задачи $(1.1),(1.2)$ или $(3.3),(3.4)$ в виде $u(x)=u_{0}(x)+u_{1}(x)$, где $u_{1}(x) \in \stackrel{\circ}{W_{2}^{1},|\mu|}\left(\Omega ; \Gamma_{1}\right)$, то подстановка такого разложения в (3.5a) или (3.5б) приводит, соответственно, $\mathrm{k}$ вариационной задаче

$$
a[u, v]_{\mu}+\int_{\Omega} \alpha(x, D) u \bar{v} d x+\int_{\Gamma_{2}} \sigma_{2} u \bar{v} d s=\langle F, \bar{v}\rangle_{\Omega}-\langle h, \bar{v}\rangle_{\Omega}
$$

или

$$
a[u, v]_{\bar{\mu}}+\int_{\Omega} u \overline{\alpha(x, D) v} d x+\int_{\Gamma_{2}} u \overline{\sigma_{2} v} d s=\langle F, \bar{v}\rangle_{\Omega}-\left\langle h^{\prime}, \bar{v}\right\rangle_{\Omega}
$$

$\forall v \in \stackrel{\circ}{W}{ }_{2,|\mu|}^{1}\left(\Omega ; \Gamma_{1}\right)$, где $\langle F, \bar{v}\rangle_{\Omega}$ определен $(3.14)$,

$$
\begin{aligned}
\langle h, v\rangle_{\Omega} & =a\left[u_{0}, \bar{v}\right]_{\mu}+\int_{\Omega} \alpha(x, D) u_{0} v d x+\int_{\Gamma_{2}} \sigma_{2} u_{0} v d s \\
\left\langle h^{\prime}, v\right\rangle_{\Omega} & =a\left[u_{0}, \bar{v}\right]_{\bar{\mu}}+\int_{\Omega} u_{0} \overline{\alpha(x, D) \bar{v}} d x+\int_{\Gamma_{2}} u_{0} \overline{\sigma_{2} \bar{v}} d s .
\end{aligned}
$$


Функционалы (3.26a) и (3.26б) являются ограниченными на $\stackrel{\circ}{W} \underset{2,|\mu|}{1}\left(\Omega ; \Gamma_{1}\right)$. Действительно, аналогично оцениванию формы $B(u, v)$ получаем

$$
\left|\langle h, v\rangle_{\Omega}\right|,\left|\left\langle h^{\prime}, v\right\rangle_{\Omega}\right| \leqslant\left(C+C_{1}|\mu|^{-1}\right)\left\|u_{0}\right\|_{W_{2,|\mu|}^{1}(\Omega)}\|v\|_{W_{2,|\mu|}^{1}(\Omega)}
$$

Поэтому, используя эти оценки с оценкой (3.24), а также оценку для функционала $F$ точно так же, как и на III-м этапе, с единственной лишш заменой $F$ на $F-h$ или $F-h^{\prime}$, соответственно, приходим к полной оценке (3.11).

Если $\mu$ - произвольное, то как и выше, найдется продолжение $u_{0}(x)$ функции $\varphi(x)$, для которого справедлива оценка вида (3.24) (с $|\mu|$ замененным, например, на $|\mu|+1)$. Условие разрешимости задачи $(1.1),(1.2)$ или (3.3), (3.4) является, соответственно, условием разрешимости вариационной задачи (3.25a) или (3.25б), которое имеет вид

$$
\left\langle F, \bar{z}^{*}\right\rangle_{\Omega}+\left\langle h, \bar{z}^{*}\right\rangle_{\Omega}=0 \quad \forall z^{*}
$$

или

$$
\langle F, \bar{z}\rangle_{\Omega}+\langle h, \bar{z}\rangle_{\Omega}=0 \quad \forall z
$$

где $z$ или $z^{*}$ - произвольное о.р. однородной задачи (1.1), (1.2) или (3.3), (3.4). Согласно (3.26a) и (3.26б), определениям 3.2 и 3.3 получаем, что

$$
\begin{aligned}
\overline{\left\langle h, \bar{z}^{*}\right\rangle_{\Omega}} & =a\left[z^{*}, u_{0}\right]_{\bar{\mu}}+\int_{\Omega} z^{*} \overline{\alpha(x, D) u_{0}} d x+\int_{\Gamma_{2}} z^{*} \overline{\sigma_{2} u_{0}} d s \\
& =\left\langle\left.\left[\frac{\partial z^{*}}{\partial \nu}+\left(\bar{\sigma}^{0}+\sum_{i=1}^{n} \bar{a}_{i} n_{i}\right) z^{*}\right]\right|_{\Gamma},\left.\bar{u}_{0}\right|_{\Gamma}\right\rangle_{\Gamma} \\
& =\left\langle\frac{\partial z^{*}}{\partial \nu}, \bar{\varphi}\right\rangle_{\Gamma_{1}}=\left\langle\frac{\partial \bar{z}^{*}}{\partial \nu}, \varphi\right\rangle_{\Gamma_{1}}
\end{aligned}
$$

и аналогично, что

$$
\left\langle h^{\prime}, \bar{z}\right\rangle_{\Omega}=\left\langle\frac{\partial \bar{z}}{\partial \nu}, \varphi\right\rangle_{\Gamma_{1}} .
$$

Последнее утверждение теоремы является непосредственным следствием утверждения, установленного перед теоремой 3.1 .

Таким образом, теорема 3.1. полностью установлена.

\section{§4. О собственных функциях эллиптического оператора со смешанными граничными условиями (самосопряженный случай)}

Рассмотрим эллиптический оператор $L(x, D)=L(x, D, 0)$, где $L(x, D, \mu)$ определен (1.3), с однородными граничными условиями вида (1.2). В дополнение к предыдущим предположениям полагаем здесь, что все $a_{i}(x) \equiv 0, a_{0}(x)$ и $\sigma(x)$ - действительные функции из $L_{\infty}(\Omega)$ и $L_{\infty}\left(\Gamma_{2}\right)$, соответственно. $\Gamma_{1}$ и $\Gamma_{2}$ предполагаются открытыми кусками $\Gamma$, удовлетворяющими условиям $\Gamma_{1} \cap \Gamma_{2}=\varnothing$, $m_{\Gamma}\left(\Gamma \backslash\left(\Gamma_{1} \cup \Gamma_{2}\right)\right)=0$.

В этом параграфе приведем с доказательством основное утверждение о разложении по обобщенным собственным функциям такого оператора в пространствах 
$L_{2}(\Omega), \stackrel{\circ}{W}=\frac{\circ}{2}\left(\Omega ; \Gamma_{1}\right)$ и $\stackrel{\circ}{W}_{2}^{-1}\left(\Omega ; \Gamma_{1}\right)$. Для случая оператора с однородными граничньми условиями Дирихле или третьего рода в областях класса $C^{1}$ доказательство базисности системы собственных функций в пространствах $L_{2}(\Omega)$ и $\stackrel{\circ}{W} \underset{2}{\stackrel{1}{W}}(\Omega)$ или $W_{2}^{1}(\Omega)$, соответственно, имеется, например, в [18, гл. 4].

ОПРЕДЕЛЕНИЕ 4.1. Обобщенная собственная функция (о.с.ф.) $e(x)$, отвечающая собственному значению (с.з.) $\lambda$, оператора $L(x, D)$ с однородными граничными условиями вида (1.2) является нетривиальным решением задачи

$$
L(x, D) e=\lambda e \text { в }\left.\Omega \quad e\right|_{\Gamma_{1}}=0,\left.\quad\left(\frac{\partial e}{\partial \nu}+\sigma e\right)\right|_{\Gamma_{2}}=0
$$

т.е. функцией, принадлежащей пространству $\stackrel{\circ}{\underset{W}{W}} \underset{2}{2}\left(\Omega ; \Gamma_{1}\right)$ и удовлетворяющей условиям $\|e\|_{L_{2}(\Omega)} \neq 0$ и

$$
a[e, v]_{0}=\lambda(e, v)_{L_{2}(\Omega)} \quad \forall v \in \stackrel{\circ}{W} \underset{2}{1}\left(\Omega ; \Gamma_{1}\right)
$$

где $a[u, v]_{0}-$ форма $(3.2 \mathrm{a})$ с $\mu=0, a_{0}^{1}(x)=a_{0}(x), \sigma_{1}(x)=\sigma(x)$.

Естественно, что $e(x)$ будет о.с.ф., отвечающей с.з. $\lambda$, оператора $L(x, D)$ с однородными граничными условиями (1.2) тогда и только тогда, когда $e(x)$ - о.с.ф. оператора $L(x, D)+\mu^{2} I, \mu>0$, с теми же граничными условиями, отвечающая с.з. $\lambda+\mu^{2}$. Таким образом,

$$
a[e, v]_{\mu}=\left(\lambda+\mu^{2}\right)(e, v)_{L_{2}(\Omega)} \quad \forall v \in \stackrel{\circ}{W_{2}^{1}}\left(\Omega ; \Gamma_{1}\right)
$$

где $a[u, v]_{\mu}$ - форма $(3.2 \mathrm{a})$ с $q(x) \equiv 1, \sigma_{1}(x)=\sigma(x)$. Поскольку при достаточно больших $\mu: \mu \geqslant \mu_{0}>0$, имеет место оценка (3.12), удобно использовать для определения о.с.ф. (4.3), а в качестве скалярного произведения в $\stackrel{\circ}{W} \underset{2, \mu}{1}\left(\Omega ; \Gamma_{1}\right)$ - форму $a[u, v]_{\mu}\left(\right.$ c $\left.q(x) \equiv 1, \sigma_{1}(x)=\sigma(x)\right)$ при каком либо $\mu \geqslant \mu_{0}$.

Далее будет существенно использоваться оператор $G_{\mu}$, определяемый по форме $a[u, v]_{\mu}$ с помощью соотношения (3.16). Поскольку $G_{\mu}$ представляет собой гомеоморфизм пространства $\stackrel{\circ}{W} \underset{2, \mu}{-1}\left(\Omega ; \Gamma_{1}\right)$ на пространство $\stackrel{\circ}{W} \underset{2, \mu}{1}\left(\Omega ; \Gamma_{1}\right)$, то в качестве скалярного произведения в $\stackrel{\circ}{W} \underset{2, \mu}{-1}\left(\Omega ; \Gamma_{1}\right)$ можно использовать форму

$$
a^{-1}\left[f_{1}, f_{2}\right]_{\mu}=a\left[G_{\mu} f_{1}, G_{\mu} f_{2}\right]_{\mu}=\left\langle f_{1}, \overline{G_{\mu} f_{2}}\right\rangle_{\Omega} \quad \forall f_{1}, f_{2} \in \stackrel{\circ}{W_{2, \mu}^{-1}}\left(\Omega ; \Gamma_{1}\right) .
$$

При этом

$$
c^{2}\|f\|_{W_{2, \mu}^{-1}\left(\Omega ; \Gamma_{1}\right)}^{2} \leqslant a^{-1}[f, f]_{\mu} \leqslant C^{2}\|f\|_{W_{2, \mu}^{-1}\left(\Omega ; \Gamma_{1}\right)}^{2}
$$

$\forall f \in \stackrel{\circ}{W}-1, \mu\left(\Omega ; \Gamma_{1}\right)$ с некоторыми постоянными $c>0, C>0$, не зависящими от $\mu$. 
ТЕОРЕма 4.1. При сформулированных выше условиях оператор $L(x, D)$ с однородными граничнымм условиями (1.2) обладает ортонормированным в $L_{2}(\Omega)$ базисом $\left\{e_{k}(x)\right\}_{k=1}^{\infty}$ из о.с.ф. Соответствующие $e_{k}(x)$ с.з. $\lambda_{k}$ действительны и стремятся $\kappa+\infty$ при $k \rightarrow \infty$ (будем считать, что $e_{k}(x)$ занумерованы в порядке неубывания $\left.\lambda_{k}: \lambda_{k} \leqslant \lambda_{k+1} \forall k \in \mathbb{N}\right)$. Системы о.с.ф.

$$
\begin{aligned}
& \left\{\left(\lambda_{k}+\mu^{2}\right)^{-1 / 2} e_{k}(x)\right\}_{k=1}^{\infty}, \\
& \left\{\left(\lambda_{k}+\mu^{2}\right)^{1 / 2} e_{k}(x)\right\}_{k=1}^{\infty}
\end{aligned}
$$

представляют собой ортонормированнье базисъ, соответственно, в пространстве $\stackrel{\circ}{\mathrm{W}} \underset{2, \mu}{1}\left(\Omega ; \Gamma_{1}\right)$ со скалярным произведением $a[u, v]_{\mu}$ и в пространстве $\stackrel{\circ}{W}-1, \mu\left(\Omega ; \Gamma_{1}\right)$ со скалярным произведением (4.4).

Всякая функция $u(x) \in \stackrel{\circ}{W} \underset{2}{1}\left(\Omega ; \Gamma_{1}\right)$ представима сходящимся в $W_{2}^{1}(\Omega)$ рядом

$$
u(x)=\sum_{k=1}^{\infty} c_{k}(u) e_{k}(x)
$$

где $c_{k}(u)=\left(u, e_{k}\right)_{L_{2}(\Omega)}-$ коэффициенть Фурье функиии и $(x)$. Для любъх и и из $\stackrel{\circ}{W}_{2, \mu}^{1}\left(\Omega ; \Gamma_{1}\right)$ справедливо равенство Парсеваля-Стеклова

$$
a[u, v]_{\mu}=\sum_{k=1}^{\infty}\left(\lambda_{k}+\mu^{2}\right) c_{k}(u) \overline{c_{k}(v)} .
$$

Всякий $f(x) \in \stackrel{\circ}{W_{2, \mu}^{-1}}\left(\Omega ; \Gamma_{1}\right)$ представимм сходящимся в $\stackrel{\circ}{W_{2}^{-1}}\left(\Omega ; \Gamma_{1}\right)$ рядом

$$
f(x)=\sum_{k=1}^{\infty} c_{k}(f) e_{k}(x)
$$

где $c_{k}(f)=\left\langle f, \bar{e}_{k}\right\rangle_{\Omega}-$ коэффициенть Фурье функиионала $f$. Для любъх $f$ и $g$ из $\stackrel{\circ}{W} \underset{2, \mu}{-1}\left(\Omega ; \Gamma_{1}\right)$ справедливо равенство Парсеваля-Стеклова

$$
a^{-1}[f, g]_{\mu}=\sum_{k=1}^{\infty}\left(\lambda_{k}+\mu^{2}\right)^{-1} c_{k}(f) \overline{c_{k}(g)}
$$

ДокАЗАТЕЛЬСТво. На основании определений (3.16) оператора $G_{\mu}$ и (4.3) элементарно проверяется, что функция $e(x) \in \stackrel{\circ}{W} \underset{2}{1}\left(\Omega ; \Gamma_{1}\right)$ является отвечающей с.з. $\lambda$ о.c.ф. оператора $L(x, D)$ с однородными граничными условиями $(1.2)$ тогда и только тогда, когда $e(x)$ - собственная функция (с.ф.) оператора $G_{\mu}$, отвечающая с.з. $\left(\lambda+\mu^{2}\right)^{-1}$. Но в силу свойств $2^{\circ}$ оператора $G_{\mu}$ как оператора в $L_{2}(\Omega)$ (компактности и положительной определенности) у оператора $G_{\mu}$ сушествует ортонормированный в $L_{2}(\Omega)$ базис $\left\{e_{k}(x)\right\}_{k=1}^{\infty}$ из с.ф.., отвечаюших собственным значениям $\gamma_{k}$, 
$\gamma_{k}>0, \gamma_{k} \rightarrow 0$ при $k \rightarrow \infty: G_{\mu} e_{k}=\gamma_{k} e_{k}$ (см., например, [30, гл. VI, п. 93]). Отсюда $\left\{e_{k}(x)\right\}_{k=1}^{\infty}-$ ортонормированньй базис в $L_{2}(\Omega)$ из о.с.ф., отвечающих с.з. $\lambda_{k}=\gamma_{k}^{-1}-\mu^{2}$ оператора $L(x, D)$ с однородньпи условиями $(1.2)$.

Далее, в силу (4.3) и (4.4)

$$
\begin{aligned}
a\left[e_{k}, e_{j}\right]_{\mu} & =\left(\lambda_{k}+\mu^{2}\right)\left(e_{k}, e_{j}\right)_{L_{2}(\Omega)}=\left(\lambda_{k}+\mu^{2}\right) \delta_{k}^{j}, \\
a^{-1}\left[e_{k}, e_{j}\right]_{\mu} & =a\left[G_{\mu} e_{k}, G_{\mu} e_{j}\right]_{\mu}=\left(\lambda_{k}+\mu^{2}\right)^{-2} a\left[e_{k}, e_{j}\right]_{\mu}=\left(\lambda_{k}+\mu^{2}\right)^{-1} \delta_{k}^{j},
\end{aligned}
$$

где $\delta_{k}^{j}$ - символ Кронекера.

Проверим, что система $\left\{e_{k}(x)\right\}_{k=1}^{\infty}$ является замкнутой как в $\stackrel{\circ}{W} \frac{1}{2, \mu}\left(\Omega ; \Gamma_{1}\right)$, так

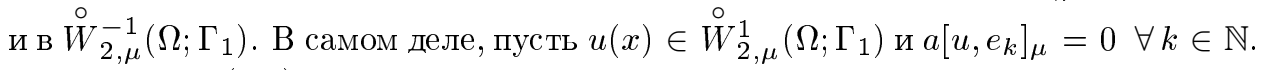
Тогда, в силу (4.3)

$$
a\left[u, e_{k}\right]_{\mu}={\overline{a\left[e_{k}, u\right.}}_{\mu}=\left(\lambda_{k}+\mu^{2}\right){\overline{\left(e_{k}, u\right)}}_{L_{2}(\Omega)}=\left(\lambda_{k}+\mu^{2}\right)\left(u, e_{k}\right)_{L_{2}(\Omega)}=0,
$$

и так как $\left(\lambda_{k}+\mu^{2}\right)>0,\left(u, e_{k}\right)_{L_{2}(\Omega)}=0 \forall k \in \mathbb{N}$. Но $\left\{e_{k}\right\}_{k=1}^{\infty}$ - замкнутая система в $L_{2}(\Omega)$, а потому последние равенства влекут, что $u(x)=0$ п.в. на $\Omega$. Таким образом, замкнута в $\stackrel{\circ}{W} \underset{2, \mu}{1}\left(\Omega ; \Gamma_{1}\right)$ и ортонормированная система $(4.6 \mathrm{a})$, а потому она образует ортонормированный базис в $\stackrel{\circ}{W} \underset{2, \mu}{1}\left(\Omega ; \Gamma_{1}\right)$.

Точно так же, пусть $f(x) \in \stackrel{\circ}{W} \underset{2, \mu}{-1}\left(\Omega ; \Gamma_{1}\right)$ и $a^{-1}\left[f, e_{k}\right]_{\mu}=0 \forall k \in \mathbb{N}$. Тогда в силу (4.4) и (3.16)

$$
a^{-1}\left[f, e_{k}\right]_{\mu}={\overline{a\left[G_{\mu} e_{k}, G_{\mu} f\right.}}_{\mu}={\overline{\left(e_{k}, G_{\mu} f\right)_{L_{2}(\Omega)}}}=\left(G_{\mu} f, e_{k}\right)_{L_{2}(\Omega)}=0 \quad \forall k \in \mathbb{N} .
$$

Отсюда $G_{\mu} f=0$, и $f=0$ в силу обратимости $G_{\mu}$. Таким образом, замкнута в $\stackrel{\circ}{W}_{2, \mu}^{-1}\left(\Omega ; \Gamma_{1}\right)$ и ортонормированная система (4.6б), а потому она образует ортонормированный базис в $\stackrel{\circ}{W} \underset{2, \mu}{-1}\left(\Omega ; \Gamma_{1}\right)$ со скалярньм произведением (4.4).

Пусть теперь функция $u(x) \in \stackrel{\circ}{W} \underset{2, \mu}{1}\left(\Omega ; \Gamma_{1}\right)$. Тогда $u(x)$ представима сходящимся в $\stackrel{\circ}{W} \underset{2, \mu}{1}\left(\Omega ; \Gamma_{1}\right)$ рядом Фурье по системе (4.6а):

$$
u(x)=\sum_{k=1}^{\infty} a\left[u,\left(\lambda_{k}+\mu^{2}\right)^{-1 / 2} e_{k}\right]_{\mu}\left(\lambda_{k}+\mu^{2}\right)^{-1 / 2} e_{k}(x) .
$$

Используя (4.3), имеем

$\left(\lambda_{k}+\mu^{2}\right)^{-1} a\left[u, e_{k}\right]_{\mu}=\left(\lambda_{k}+\mu^{2}\right)^{-1}{\overline{a\left[e_{k}, u\right]_{\mu}}}_{\mu}={\overline{\left(e_{k}, u\right)}}_{L_{2}(\Omega)}=\left(u, e_{k}\right)_{L_{2}(\Omega)}=c_{k}(u)$, а потому ряд (4.10a) совпадает с рядом в правой части (4.7a).

Точно так же, всякий функционал $f \in \stackrel{\circ}{W_{2, \mu}^{-1}}\left(\Omega ; \Gamma_{1}\right)$ представи́м сходящимся в $\stackrel{\circ}{W} \underset{2, \mu}{-1}\left(\Omega ; \Gamma_{1}\right)$ рядом Фурье в этом пространстве по системе (4.6б):

$$
f(x)=\sum_{k=1}^{\infty} a^{-1}\left[f,\left(\lambda_{k}+\mu^{2}\right)^{1 / 2} e_{k}\right]_{\mu}\left(\lambda_{k}+\mu^{2}\right)^{1 / 2} e_{k}(x) .
$$


Воспользовавшись $(4.4),(3.16)$ и равенствами $\left(\lambda_{k}+\mu^{2}\right) G_{\mu} e_{k}=e_{k}$, находим, что

$$
\left(\lambda_{k}+\mu^{2}\right) a^{-1}\left[f, e_{k}\right]_{\mu}=\left(\lambda_{k}+\mu^{2}\right) a\left[G_{\mu} f, G_{\mu} e_{k}\right]_{\mu}=\left\langle f, \bar{e}_{k}\right\rangle_{\Omega}=c_{k}(f),
$$

а потому ряд (4.10б) совпадает с рядом в правой части (4.7б).

Наконец, равенства Парсеваля-Стеклова (4.8а) и (4.8б) получаются подстановкой в формы $a[u, v]_{\mu}$ и $a^{-1}[f, g]_{\mu}$ разложений элементов в ряды Фурье при использовании соотношений ортогональности (4.9a) и (4.9б).

По поводу о.р. краевых задач и разложениям по о.с.ф. в самосопряженном случае см. также [32]. По поводу разложений по с.ф. сильно эллиптических несамосопряженных операторов с однородньми условиями Дирихле или Неймана см., например, [33].

\section{§5. Об операторах Пуанкаре-Стеклова}

В настоящем параграф̆е будет рассмотрено обобщение развитой в [15] и [16] теории операторов Пуанкаре-Стеклова на куске $\gamma$ границы $Г$ для того случая, когда на оставшейся части $\Gamma \backslash \bar{\gamma}$ задаются однородные смешанные граничные условия.

Предположим, что граница Г липшицевой области $\Omega$ разбита на три открытых непересекающихся куска $\gamma, \Gamma_{1}$ и $\Gamma_{2}$ в том смысле, что $m_{\Gamma}\left(\Gamma \backslash\left(\gamma \cup \Gamma_{1} \cup \Gamma_{2}\right)\right)=0$. Последнее означает, что $\Gamma=\gamma \cup \Gamma_{1} \cup \Gamma_{2} \cup \delta$, где $\gamma, \Gamma_{1}, \Gamma_{2}$ и $\delta$ попарно не пересекаются и $m_{\Gamma}(\delta)=0$. Рассмотрим граничную задачу:

$$
\begin{aligned}
& L(x, D, \mu) u(x)=0 \text { в } \Omega \\
& \left.u(x)\right|_{\Gamma_{1}}=0,\left.\quad\left(\frac{\partial u}{\partial \nu}(x)+\sigma(x) u(x)\right)\right|_{\Gamma_{2}}=0,\left.\quad \frac{\partial u}{\partial \nu}(x)\right|_{\gamma}=g(x),
\end{aligned}
$$

где $L(x, D, \mu)$ - равномерно эллиптический оператор (1.3). Вначале предположения о коэффициентах уравнения, $\sigma(x)$ и значениях параметра $\mu$ остаются теми же, что в $\oint 1$ и в начале $\S 3$. Рассмотрения этого параграфа будут существенно использованы в следуюшем параграфе.

Задачу (5.1), (5.2) будем рассматривать, в соответствии с предыдушей теорией, как смешанную задачу для уравнения (5.1) с граничньми условиями

$$
\left.u\right|_{\Gamma_{1}}=0,\left.\quad\left(\frac{\partial u}{\partial \nu}(x)+\stackrel{\circ}{\sigma}(x) u(x)\right)\right|_{\Gamma_{3}}=\stackrel{\circ}{g}(x)
$$

где $\Gamma_{3}=\Gamma \backslash \bar{\Gamma}_{1}$

$$
\stackrel{\circ}{\sigma}(x)= \begin{cases}\sigma(x) & \text { при } x \in \Gamma_{2}, \\ 0 & \text { при } x \in \Gamma_{3} \backslash \Gamma_{2},\end{cases}
$$

$\stackrel{\circ}{g}(x)$ - функционал из $\stackrel{\circ}{W}_{2,|\mu|}^{-1 / 2}\left(\Gamma_{3}\right)$ с носителем на $\bar{\gamma}$, т.е. $\stackrel{\circ}{g}(x)$ обращается в нуль на $\Gamma_{2}$ :

$$
\langle\stackrel{\circ}{g}, \psi\rangle_{\Gamma_{3}}=0 \quad \forall \psi \in \stackrel{\stackrel{1}{W}}{1 / 2},|\mu|\left(\Gamma_{2}\right),
$$


и при этом $\stackrel{\circ}{g}(x)$ совпадает на $\gamma$ с $g(x)$ в определенном смысле. Нам будет для дальнейшего удобно дать эквивалентное описание таких функционалов $\stackrel{\circ}{g}(x)$ через их значения $g(x)$ на $\gamma$, описываемые как функционалы над некоторым пространством функций, определенных только на $\gamma$.

Обозначим $\stackrel{\stackrel{1}{W}}{2, \mu}\left(\gamma \rightarrow \Gamma ; \Gamma_{1}\right), \mu>0$, факторпространство $\stackrel{\circ}{W} \underset{2, \mu}{1 / 2}\left(\Gamma_{3}\right) / \stackrel{\circ}{W_{2, \mu}^{1 / 2}}\left(\Gamma_{2}\right)$, т.е. подпространство функций $\varphi(x)$ из $W_{2, \mu}^{1 / 2}(\gamma)$, допускающих продолжения $l \varphi \in$ $\stackrel{\circ}{W} \underset{2, \mu}{1 / 2}\left(\Gamma_{3}\right)$, с нормой в этом пространстве, определяемой выражением

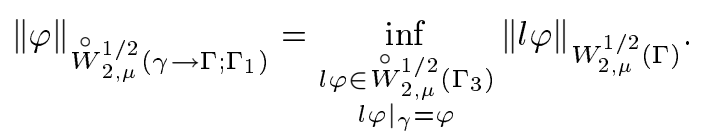

Пространство $\stackrel{\circ}{W} \underset{2, \mu}{1 / 2}\left(\gamma \rightarrow \Gamma ; \Gamma_{1}\right)$ является (как факторпространство банаховых) банаховым пространством (см., например, [31]). Обозначим $\stackrel{\circ}{W_{2, \mu}^{-1 / 2}}\left(\gamma \rightarrow \Gamma ; \Gamma_{1}\right)$ пространство, сопряженное пространству $\stackrel{\circ}{W} \underset{2, \mu}{1 / 2}\left(\gamma \rightarrow \Gamma ; \Gamma_{1}\right)$.

ЛЕмма 5.1. Существует естественнъй изометрический изоморфизм между подпространством функиионалов из $\stackrel{\circ}{\underset{2}{2}-\mu}\left(\Gamma_{3}\right)$ с носителями в $\bar{\gamma}$ и пространством $\left.\stackrel{\circ}{W}-1 / 2, \gamma \rightarrow \Gamma ; \Gamma_{1}\right)$. А именно, всякому функционалу $\stackrel{\circ}{g}(x) \in$ $\stackrel{\circ}{W_{2, \mu}^{-1 / 2}}\left(\Gamma_{3}\right)$ с носителем в $\bar{\gamma}$ можно поставить во взаимно однозначное соответствие функционал $g(x) \in \stackrel{\circ}{W_{2, \mu}^{-1 / 2}}\left(\gamma \rightarrow \Gamma ; \Gamma_{1}\right)$ так, что при этом

$$
\langle g, \varphi\rangle_{\gamma}=\langle\stackrel{\circ}{g}, l \varphi\rangle_{\Gamma_{3}},
$$

где $l \varphi \in \stackrel{\circ}{W_{2, \mu}^{1 / 2}}\left(\Gamma_{3}\right)$ - произвольное продолжение $\varphi \in \stackrel{\circ}{\stackrel{1}{W}_{2, \mu}^{1 / 2}}\left(\gamma \rightarrow \Gamma ; \Gamma_{1}\right)$, и имеет место равенство

$$
\|g\|_{\stackrel{\circ}{2, \mu}_{2,1 / 2}^{\left.-1 / \gamma \rightarrow \Gamma ; \Gamma_{1}\right)}}=\|\stackrel{\circ}{g}\|_{\stackrel{\circ}{2, \mu}_{2,1 / 2}^{-1}\left(\Gamma_{3}\right)} .
$$

ДокАЗАТЕЛЬСтво. Пусть $\stackrel{\circ}{g}(x) \in \stackrel{\circ}{W_{2, \mu}^{-1 / 2}}\left(\Gamma_{3}\right)$, supp $\stackrel{\circ}{g}(x) \subseteq \bar{\gamma}$. Тогда значение правой части в (5.7) в силу (5.5) не зависит от продолжения $l \varphi$ элемента $\varphi \in$ $\stackrel{\circ}{W}_{2, \mu}^{1 / 2}\left(\gamma \rightarrow \Gamma ; \Gamma_{1}\right)$, а потому формула (5.7) определяет линейный функционал на этом пространстве. Кроме того,

$$
\left|\langle g, \varphi\rangle_{\gamma}\right|=\left|\langle\stackrel{\circ}{g}, l \varphi\rangle_{\Gamma_{3}}\right| \leqslant\|\stackrel{\circ}{g}\|_{\stackrel{\circ}{2, \mu}_{2,1 / 2}^{\left.-1 / \Gamma_{3}\right)}} \cdot\|l \varphi\|_{W_{2, \mu}^{1 / 2}\left(\Gamma_{3}\right)} .
$$

Переходя в этой оценке к inf по продолжениям $l \varphi$ получим, что левая часть в $(5.8)$ не превосходит правой части.

Пусть задан $g(x) \in \stackrel{\circ}{{ }^{2}} \underset{2, \mu}{-1 / 2}\left(\gamma \rightarrow \Gamma ; \Gamma_{1}\right)$. Определим функционал $\stackrel{\circ}{g}(x)$ формулой (5.7), а более точно, действие $\stackrel{\circ}{g}(x)$ на произвольную функцию $\psi \in \stackrel{\circ}{W_{2, \mu}^{1 / 2}}\left(\Gamma_{3}\right)$ определим по формуле

$$
\langle\stackrel{\circ}{g}, \psi\rangle_{\Gamma_{3}}=\left\langle g,\left.\psi\right|_{\gamma}\right\rangle_{\gamma}
$$


Очевидно выполняется (5.5) и, кроме того,

$$
\left|\langle\stackrel{\circ}{g}, \psi\rangle_{\Gamma_{3}}\right|=\left|\left\langle g,\left.\psi\right|_{\gamma}\right\rangle_{\gamma}\right| \leqslant\|g\|_{W_{2, \mu}^{-1 / 2}\left(\gamma \rightarrow \Gamma ; \Gamma_{1}\right)} \cdot\|\psi\|_{W_{2, \mu}^{1 / 2}\left(\Gamma_{3}\right)},
$$

откуда следует, что правая часть в (5.8) не превосходит левую. Легко также убедиться, что введенные только что операции $\stackrel{\circ}{g} \rightarrow g$ и $g \rightarrow \stackrel{\circ}{g}$ действительно являются взаимно обратньми.

Итак, в задаче (5.1), (5.2) будем предполагать, что $g(x) \in \stackrel{\circ}{\underset{W}{2}-1 / 2}\left(\gamma \rightarrow \Gamma ; \Gamma_{1}\right)$, а саму эту задачу понимать как задачу $(5.1),(5.3)$ с $\stackrel{\circ}{g}(x) \in \stackrel{\circ}{W_{2,|\mu|}^{-1 / 2}}\left(\Gamma_{3}\right)$, определяемым по $g(x)$ согласно соответствию, устанавливаемому леммой 5.1. Будем предполагать также, что задача (5.1), (5.3) однозначно разрешима, например, ограничимся для определенности рассмотрением случая $\operatorname{Re} \mu \geqslant \mu_{*}$ (см. теоремy 4.1).

ОПРЕДЕЛЕНИЕ 5.1. Линейный оператор $S_{\mu}$, определенный на любом функционале $g \in \stackrel{\circ}{W}-1 / 2\left(\gamma \mid\left(\gamma \rightarrow \Gamma ; \Gamma_{1}\right)\right.$ по формуле

$$
S_{\mu} g=\left.u\right|_{\gamma},
$$

где $u(x) \in \stackrel{\circ}{W} \frac{1}{2,|\mu|}\left(\Omega ; \Gamma_{1}\right)$ - решение задачи $(5.1),(5.2)$, назовем оператором Пуанкаре-Стеклова, соответствуюшим этой задаче (см. [15], [16]).

Теорема 5.1. При $\operatorname{Re} \mu \geqslant \mu_{*}$, где $\mu_{*}$ - значение, появляющееся в теореме 4.1, оператор $S_{\mu}$ представляет собой гомеоморфизм пространства $\stackrel{\circ}{W}-1 / 2\left(\gamma \rightarrow \Gamma ; \Gamma_{1}\right)$ на пространство $\stackrel{\circ}{W_{2,|\mu|}^{1 / 2}}\left(\gamma \rightarrow \Gamma ; \Gamma_{1}\right)$. При этом, если еще $\mu$ подчинено, например, ограничению $|\arg \mu|<(\pi / 2)-\delta, \delta>0$, то нормы $S_{\mu}$ и $S_{\mu}^{-1}$ могут быть оценены постоянныцми, не зависящими от $\mu$. Сужение оператора $S_{\mu}$ на $L_{2}(\gamma)$, рассматриваемое как оператор в $L_{2}(\gamma)$, является вполне непрерывным оператором.

ДокАЗАТЕЛЬСтво. Так как решение $u(x)$ задачи $(5.1),(5.3)$ принадлежит про-

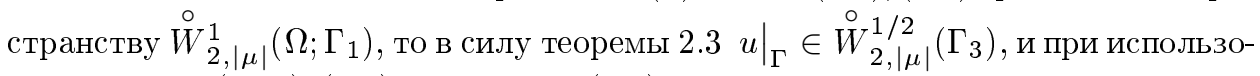
вании оценок $(2.13),(3.1)$ и равенства $(5.8)$ получаем

$$
\begin{aligned}
\left\|S_{\mu} g\right\|_{\stackrel{\circ}{2}_{2,|\mu|}^{1 / 2}\left(\gamma \rightarrow \Gamma ; \Gamma_{1}\right)} & \leqslant\|u\|_{\stackrel{\circ}{2,|\mu|}_{1 / 2}^{1 / 2}\left(\Gamma_{3}\right)} \leqslant c_{0}(\Omega, 1)\|u\|_{W_{2,|\mu|}^{1}(\Omega)} \\
& \leqslant C(1+|\operatorname{Im} \mu| / \operatorname{Re} \mu)\|g\|_{W_{2,|\mu|}^{-1 / 2}\left(\gamma \rightarrow \Gamma ; \Gamma_{1}\right)} .
\end{aligned}
$$

Чтобы получить обратимость $S_{\mu}$ и оценку нормы $S_{\mu}^{-1}$, рассмотрим для произвольной $\varphi(x) \in \stackrel{\circ}{\stackrel{1}{W}} 2,|\mu|\left(\gamma \rightarrow \Gamma ; \Gamma_{1}\right)$ смешанную задачу для уравнения $(5.1) \mathrm{c}$ граничньм условием

$$
\left.u\right|_{\Gamma_{1}}=0,\left.\quad u\right|_{\gamma}=\varphi,\left.\quad\left(\frac{\partial u}{\partial \nu}+\sigma u\right)\right|_{\Gamma_{2}}=0
$$


Обозначим

$$
\stackrel{\circ}{\varphi}(x)= \begin{cases}\varphi(x) & \text { при } x \in \gamma, \\ 0 & \text { при } x \in \Gamma_{1} .\end{cases}
$$

Легко видеть, что

$$
\|\stackrel{\circ}{\varphi}\|_{W_{2,|\mu|}^{1 / 2}\left(\Gamma \backslash \bar{\Gamma}_{2}\right)} \leqslant\|\varphi\|_{\stackrel{\circ}{2}_{2,|\mu|}^{1 / 2}\left(\gamma \rightarrow \Gamma ; \Gamma_{1}\right)},
$$

а потому (это можно установить, проанализировав вывод оценки (3.11)) для решения $u(x)$ задачи (5.1), (5.12) справедлива оценка (3.11) с $f=0, g=0$ и с $\varphi$, замененной на $\stackrel{\circ}{\varphi}, \Gamma_{1}$, замененным на $\Gamma \backslash \bar{\Gamma}_{2}$. Далее, на основании определения 3.2 можно построить по формуле (3.7a) с $\widehat{f}=0$ функционал

$$
\left.\left(\frac{\partial u}{\partial \nu}+\sigma^{0} u\right)\right|_{\Gamma} \in W_{2,|\mu|}^{-1 / 2}(\Gamma)
$$

где коэффициент $\sigma^{0}$ определен (3.6). Используя в (3.7a) $l \psi=l_{\Gamma}^{\Omega} \psi$, где $l_{\Gamma}^{\Omega}-$ оператор продолжения, фигурирующий в теореме 2.4 (соответствующий значению параметра $|\mu|)$, и производя оценки, аналогичные уже проводившимся, получаем, что

$$
\left\|\left.\left(\frac{\partial u}{\partial \nu}+\sigma^{0} u\right)\right|_{\Gamma}\right\|_{W_{2,|\mu|}^{-1 / 2}(\Gamma)} \leqslant C\|u\|_{W_{2,|\mu|}^{1}(\Omega)}
$$

с постоянной $C$, не зависящей от $\mu$. Кроме того, из предыдушего следует, что функционал

$$
\left.\left(\frac{\partial u}{\partial \nu}+\sigma^{0} u\right)\right|_{\Gamma}
$$

обрашается в нуль на $\Gamma_{2}$. Обозначим через $\stackrel{\circ}{g}(x)$ сужение этого функционала на подпространство $\stackrel{\circ}{W} \underset{2,|\mu|}{1 / 2}\left(\Gamma_{3}\right)$. Поскольку носитель $\stackrel{\circ}{g}(x)$ сосредоточен на $\bar{\gamma}$, можно построить функционал $g(x) \in \stackrel{\circ}{\underset{W}{2}}-1 / 2 \mid\left(\gamma \rightarrow \Gamma ; \Gamma_{1}\right)$, удовлетворяющий (5.7). С использованием (5.8) легко получаем, что

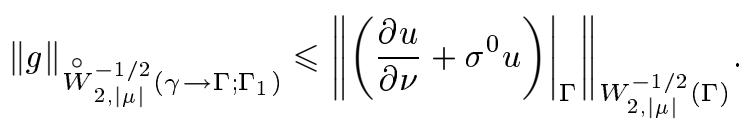

Все это приводит окончательно к оценке

$$
\|g\|_{\stackrel{\circ}{W}_{2,|\mu|}^{-1 / 2}\left(\gamma \rightarrow \Gamma ; \Gamma_{1}\right)} \leqslant C(1+|\operatorname{Im} \mu| / \operatorname{Re} \mu)\|\varphi\|_{W_{2,|\mu|}^{1 / 2}\left(\gamma \rightarrow \Gamma ; \Gamma_{1}\right)}
$$

с постоянной $C$, также не зависящей от $\mu$.

Далее, нетрудно проверить, что решение $u(x)$ задачи $(5.1),(5.2)$ с таким образом найденным по $\varphi(x)$ функционалом $g(x)$, совпадает с решением $u(x)$ задачи $(5.1),(5.12)$. Поэтому $S_{\mu} g=\varphi$. Это дает, что $S_{\mu}$ отображает на все $\stackrel{\circ}{W} \underset{2,|\mu|}{1 / 2}\left(\gamma \rightarrow \Gamma ; \Gamma_{1}\right)$

Осталось еше проверить, что $S_{\mu}$ обратим. В самом деле, если $S_{\mu} g=0$, то решение $u$ задачи (5.1), (5.2) является в то же время решением задачи (5.1), (5.12) 
с $\varphi=0$, а потому в силу единственности решения этой задачи $u=0$. Тогда в силу (3.7а) и равенства

$$
\stackrel{\circ}{g}=\left.\left(\frac{\partial u}{\partial \nu}+\sigma^{0} u\right)\right|_{\Gamma_{3}}
$$

получаем, что $\stackrel{\circ}{g}=0$, а потому и $g=0$. Оценку нормы $S_{\mu}^{-1}$ получаем при подстановке в (5.13) $g=S_{\mu}^{-1} \varphi$.

Утверждение о полной непрерывности $S_{\mu}$, рассматриваемого на $L_{2}(\gamma)$, получается аналогично соответствуюшему утверждению для оператора $G_{\mu}$.

Ниже спектральные свойства оператора $S_{\mu}$ рассмотрим (как и в $\left.[15],[16]\right)$ только для самосопряженного случая, т.е. для случая, когда коэффициенты $a_{i}(x)=0$, $i=1, \ldots, n$, коэффициенты $a_{0}(x)$ и $\sigma(x)$ - действительные функции из $L_{\infty}(\Omega)$ и $L_{\infty}\left(\Gamma_{2}\right)$, соответственно, $\mu \geqslant \mu_{0}>0$, где $\mu_{0}$ появляется на I-м этапе доказательства теоремы 3.1. Через $a[u, v]_{\mu}$ будем обозначать форму $(3.2 \mathrm{a})$ с $a_{0}^{1}(x)=a_{0}(x)$ и $\sigma_{1}(x)=\sigma(x)$. Итак, $\mu_{0}>0$ таково, что выполняется оценка (3.12) (с постоянными $c$ и $C$ не зависяшими от $\mu)$. Основными для дальнейшего являются следующие соотношения.

ЛЕмма 5.2. 1. Пусть для $g_{i} \in \stackrel{\circ}{W_{2, \mu}^{-1 / 2}}\left(\gamma \rightarrow \Gamma ; \Gamma_{1}\right)$ функиии $u_{i}(x) \in \stackrel{\circ}{W} \frac{1}{2, \mu}\left(\Omega ; \Gamma_{1}\right)$ представляют собой решения задачи (5.1), (5.2) с $g=g_{i}, i=1,2$. Тогда

$$
a\left[u_{1}, u_{2}\right]_{\mu}=\left\langle g_{1}, \overline{S_{\mu} g_{2}}\right\rangle_{\gamma}
$$

и в качестве әквивалентного скалярного произведения в $\stackrel{\circ}{W_{2, \mu}^{-1 / 2}}\left(\gamma \rightarrow \Gamma ; \Gamma_{1}\right)$ можсно принять форму (5.14).

2. Пусть для $\varphi_{i} \in \stackrel{\circ}{W} \underset{2, \mu}{1 / 2}\left(\gamma \rightarrow \Gamma ; \Gamma_{1}\right)$ функиии $u_{i}(x) \in \stackrel{\circ}{W} \underset{2, \mu}{1}\left(\Omega ; \Gamma_{1}\right)$ представляют собой решения задачи (5.1), (5.12) с $\varphi=\varphi_{i}, i=1,2$. Тогда

$$
a\left[u_{1}, u_{2}\right]_{\mu}=\left\langle S_{\mu}^{-1} \varphi_{1}, \bar{\varphi}_{2}\right\rangle_{\gamma}
$$

и в качестве әквивалентного скалярного произведения в $\stackrel{\circ}{W_{2, \mu}^{1 / 2}}\left(\gamma \rightarrow \Gamma ; \Gamma_{1}\right)$ мохсно принять форму (5.15).

ДокАЗАТЕЛЬСтво. Полагая в (3.5a) (с $\left.\sigma_{2}=0\right) u=u_{1}, v=u_{2}$, пользуясь определением (5.7) и равенством $\left.u_{2}\right|_{\gamma}=S_{\mu} g_{2}$, приходим к (5.14). Соотношение (5.15) получается из (5.14) при введении функционалов $g_{i}=S_{\mu}^{-1} \varphi_{i}$ и использовании уже установленного факта, что решения $u_{i}(x)$ задачи $(5.1),(5.2) \mathrm{c} g$, равным таким $g_{i}$, совпадают с решениями задачи (5.1), (5.12) с $\varphi=\varphi_{i}, i=1,2$.

Далее, формы (5.14) и (5.15) являются эрмитовыми. Более того, в силу теоремы 5.1, теоремы 2.3 вложения, оценки (3.12) и, наконец, оценки (3.11), имеем для решения $u(x)$ задачи $(5.1),(5.2)$

$$
\begin{aligned}
\|g\|_{W_{2, \mu}^{-1 / 2}\left(\gamma \rightarrow \Gamma ; \Gamma_{1}\right)}^{2} & \sim\left\|S_{\mu} g\right\|_{W_{2, \mu}^{1 / 2}\left(\gamma \rightarrow \Gamma ; \Gamma_{1}\right)}^{2} \leqslant\|u\|_{W_{2, \mu}^{1 / 2}(\Gamma)}^{2} \leqslant c_{0}^{2}(\Omega, 1)\|u\|_{W_{2, \mu}^{1}(\Omega)}^{2}(\Omega) \\
& \sim a[u, u]_{\mu} \leqslant C\|g\|_{W_{2, \mu}^{-1 / 2}\left(\gamma \rightarrow \Gamma ; \Gamma_{1}\right)}^{2} \cdot
\end{aligned}
$$


Если теперь $u(x)$ - решение задачи $(5.1),(5.12)$, то $u(x)$ является также и решением задачи (5.1), (5.2) с $g=S_{\mu}^{-1} \varphi$. Поэтому, пользуясь предыдущим и теоремой 5.1 , получим

$$
\begin{aligned}
\left\langle S_{\mu}^{-1} \varphi, \varphi\right\rangle_{\gamma} & =\left\langle g, S_{\mu} g\right\rangle_{\gamma} \sim\|g\|_{W_{2, \mu}^{-1 / 2}\left(\gamma \rightarrow \Gamma ; \Gamma_{1}\right)}^{2} \\
& =\left\|S_{\mu}^{-1} \varphi\right\|_{W_{2, \mu}^{-1 / 2}\left(\gamma \rightarrow \Gamma ; \Gamma_{1}\right)}^{2} \sim\|\varphi\|_{W_{2, \mu}^{1 / 2}\left(\gamma \rightarrow \Gamma ; \Gamma_{1}\right)}^{2} .
\end{aligned}
$$

Здесь под эквивалентностью понимается наличие двусторонней оценки с положительными постоянными, не зависящими от $\mu$.

В связи с этим используем следующие обозначения для этих скалярных произведений:

$$
\begin{aligned}
\left(g_{1}, g_{2}\right)_{\stackrel{\circ}{{ }^{\circ}-1 / 2}}^{*}\left(\gamma \rightarrow \Gamma ; \Gamma_{1}\right) & =\left\langle g_{1}, \overline{S_{\mu} g_{2}}\right\rangle_{\gamma}, \\
\left(\varphi_{1}, \varphi_{2}\right)_{W_{2, \mu}^{\circ}}^{*}\left(\gamma \rightarrow \Gamma ; \Gamma_{1}\right) & =\left\langle S_{\mu}^{-1} \varphi_{1}, \bar{\varphi}_{2}\right\rangle_{\gamma} .
\end{aligned}
$$

ТЕОРема 5.2. При сформулированных выше условиях самосопряженности задачи (5.1), (5.2) и условиях на параметр $\mu$, ограничение оператора $S_{\mu}$ на $L_{2}(\gamma)$ представляет собой самосопряженный вполне непрерывный неотрицательный оператор в $L_{2}(\gamma)$, у которого 0 не является собственным значением, а потому, он обладает ортонормированным базисом $\left\{\varepsilon_{k}(x)\right\}_{k=1}^{\infty}$ в $L_{2}(\gamma)$ из с.ф.:

$$
S_{\mu} \varepsilon_{k}=\nu_{k}^{-1} \varepsilon_{k}
$$

где $\nu_{k}>0, \nu_{k} \leqslant \nu_{k+1}, k \in \mathbb{N}, \nu_{k} \rightarrow+\infty n p u k \rightarrow \infty, \varepsilon_{k}(x) \in \stackrel{\circ}{W_{2, \mu}^{1 / 2}}\left(\gamma \rightarrow \Gamma ; \Gamma_{1}\right)$ ( $\nu_{k}$ и $\varepsilon_{k}$, естественно, зависят и от $\mu$ ). Системы с.ф.

$$
\begin{aligned}
& \left\{\nu_{k}^{-1 / 2} \varepsilon_{k}(x)\right\}_{k=1}^{\infty}, \\
& \left\{\nu_{k}^{1 / 2} \varepsilon_{k}(x)\right\}_{k=1}^{\infty}
\end{aligned}
$$

образуют ортонормированнье базисы, соответственно, в пространствах $\stackrel{\circ}{W}_{2, \mu}^{1 / 2}\left(\gamma \rightarrow \Gamma ; \Gamma_{1}\right)$ со скалярныц произведением (5.16a) $и \stackrel{\circ}{W_{2, \mu}^{-1 / 2}}\left(\gamma \rightarrow \Gamma ; \Gamma_{1}\right)$ со скалярныц произведением (5.16б).

Всякая $\varphi(x) \in \stackrel{\circ}{W_{2, \mu}^{1 / 2}}\left(\gamma \rightarrow \Gamma ; \Gamma_{1}\right)$ представима сходящимся в $\stackrel{\circ}{W_{2, \mu}^{1 / 2}}\left(\gamma \rightarrow \Gamma ; \Gamma_{1}\right)$ рядом

$$
\varphi(x)=\sum_{k=1}^{\infty} c_{k}(\varphi) \varepsilon_{k}(x),
$$

где $c_{k}(\varphi)=\left(\varphi, \varepsilon_{k}\right)_{L_{2}(\gamma)}-$ коэффициенты Фурье функции $\varphi$. Для любых $\varphi_{1}$ и $\varphi_{2}$ из $\stackrel{\circ}{W} \underset{2, \mu}{1 / 2}\left(\gamma \rightarrow \Gamma ; \Gamma_{1}\right)$ справедливо равенство Парсеваля-Стеклова

$$
\left(\varphi_{1}, \varphi_{2}\right)_{W_{2, \mu}^{\circ}\left(\gamma \rightarrow \Gamma ; \Gamma_{1}\right)}^{*}=\sum_{k=1}^{\infty} \nu_{k} c_{k}\left(\varphi_{1}\right) \overline{c_{k}\left(\varphi_{2}\right)} .
$$


Всякий $g \in \stackrel{\circ}{W_{2, \mu}^{-1 / 2}}\left(\gamma \rightarrow \Gamma ; \Gamma_{1}\right)$ представиім сходящимся в $\stackrel{\circ}{W_{2, \mu}^{-1 / 2}}\left(\gamma \rightarrow \Gamma ; \Gamma_{1}\right)$ рядом

$$
g(x)=\sum_{k=1}^{\infty} c_{k}(g) \varepsilon_{k}(x)
$$

где $c_{k}(g)=\left\langle g, \bar{\varepsilon}_{k}\right\rangle_{\gamma}-$ коэффициенты Фурье функционала $g$. Для любъх $g_{1}$ и $g_{2}$ из $\stackrel{\circ}{W}_{2, \mu}^{-1 / 2}\left(\gamma \rightarrow \Gamma ; \Gamma_{1}\right)$ справедливо равенство Парсеваля-Стеклова

$$
\left(g_{1}, g_{2}\right)_{\substack{W_{2, \mu}^{-1 / 2} \\\left(\gamma \rightarrow \Gamma ; \Gamma_{1}\right)}}^{*}=\sum_{k=1}^{\infty} \nu_{k}^{-1} c_{k}\left(g_{1}\right) \overline{c_{k}\left(g_{2}\right)} .
$$

ДоказАТЕЛьство. Компактность оператора $S_{\mu}$ как оператора из $L_{2}(\gamma)$ в $L_{2}(\gamma)$ уже установлена (см. теорему 5.1).

Далее, пусть $g_{i}(x) \in L_{2}(\gamma)$ - произвольные, а $u_{i}(x) \in \stackrel{\circ}{W_{2}^{1}} \underset{2, \mu}{ }\left(\Omega ; \Gamma_{1}\right)$ - решения задачи (5.1), (5.2) с $g(x)=g_{i}(x), i=1,2$. Тогда в силу леммы 5.2.1, а именно равенства (5.14),

$$
\begin{aligned}
\left(g_{1}, S_{\mu} g_{2}\right)_{L_{2}(\Omega)} & =\left\langle g_{1}, \overline{S_{\mu} g_{2}}\right\rangle_{\Omega}=a\left[u_{1}, u_{2}\right]_{\mu} \\
& =\overline{a\left[u_{2}, u_{1}\right]_{\mu}}=\overline{\left\langle g_{2}, \overline{S_{\mu} g_{1}}\right\rangle_{\Omega}}=\left(S_{\mu} g_{1}, g_{2}\right)_{L_{2}(\Omega)}
\end{aligned}
$$

Отсюда и из положительности формы $a[u, v]_{\mu}$ следует самосопряженность и неотрицательность оператора $S_{\mu}$ в $L_{2}(\gamma)$. Если же для некоторой $g \in L_{2}(\gamma) S_{\mu} g=0$, то в силу теоремы $5.1 g=0$ как элемент пространства $\stackrel{\circ}{W} \underset{2, \mu}{-1 / 2}\left(\gamma \rightarrow \Gamma ; \Gamma_{1}\right)$, что означает

$$
\int_{\gamma} g \varphi d x=0
$$

$\forall \varphi \in \stackrel{\circ}{W} \underset{2, \mu}{1 / 2}\left(\gamma \rightarrow \Gamma ; \Gamma_{1}\right)$, а потому и $\forall \varphi \in \stackrel{\circ}{W}_{2, \mu}^{1 / 2}(\gamma)$. Но тогда в силу обобшения леммы Дюбуа-Реймона для этого случая (такое обобщение может быть легко установлено с помощью проведения локализации и использования, например, леммы 2.3), получаем, что $g(x)=0$ п.в. на $\gamma$.

Далее доказательство теоремы 5.2 совершенно аналогично доказательству соответствующей части теоремы 4.1. В самом деле, в теореме 4.1 устанавливается утверждение о разложении по собственнып функциям сужения в $L_{2}(\Omega)$ (вполне непрерьвного и положительного) оператора $G_{\mu}$ в пространствах $\stackrel{\circ}{W} \frac{1}{2, \mu}\left(\Omega ; \Gamma_{1}\right)$ и $\stackrel{\circ}{W}_{2, \mu}^{-1}\left(\Omega ; \Gamma_{1}\right)$, в которых скалярные произведения вводились по сути дела по формулам $\left\langle G_{\mu}^{-1} u_{1}, \bar{u}_{2}\right\rangle_{\Omega}$ и $\left\langle f, \overline{G_{\mu} f_{2}}\right\rangle_{\Omega}$, соответственно. Настоящая же теорема представляет собой аналогичное утверждение о разложении по собственным функциям сужения в $L_{2}(\gamma)$ (вполне непрерывного и положительного) оператора $S_{\mu}$ в пространствах $\stackrel{\circ}{W} \underset{2, \mu}{1 / 2}\left(\gamma \rightarrow \Gamma ; \Gamma_{1}\right)$ и $\stackrel{\circ}{W}-1 / 2\left(\gamma \rightarrow \Gamma ; \Gamma_{1}\right)$, в которых скалярные произведения вводятся по формулам (5.16а) и (5.16б), соответственно. В связи с этим доказательство оставшейся части теоремы 5.2 опустим. 
Отметим, что оператор $S_{\mu}$ переводит базис $(5.18 \mathrm{a})$ в $\stackrel{\circ}{{ }^{2}} \underset{2, \mu}{-1 / 2}\left(\gamma \rightarrow \Gamma ; \Gamma_{1}\right)$, ортонормированный относительно скалярного произведения (5.16a), а базис (5.18б) в $\stackrel{\circ}{W} \underset{2, \mu}{1 / 2}\left(\gamma \rightarrow \Gamma ; \Gamma_{1}\right)$, ортонормированный относительно скалярного произведения (5.16б).

ОПРеДЕЛЕниЕ 5.2. Решение $E_{k}(x) \in \stackrel{\circ}{W} \frac{1}{2, \mu}\left(\Omega ; \Gamma_{1}\right)$ задачи $(5.1),(5.2)$ с $g(x)=$ $\nu_{k}^{1 / 2} \varepsilon_{k}(x)$ или, что то же самое, решение задачи $(5.1),(5.12)$ с $\varphi(x)=\nu_{k}^{-1 / 2} \varepsilon_{k}(x)$ назовем фундаментальной функиией (ф.ф.) Пуанкаре-Стеклова, соответствующей с.ф.. $\varepsilon_{k}(x)$ оператора $S_{\mu}$ (см. [15], [16]).

С помошью разложений функционала $g$ в граничном условии в (5.2) или функции $\varphi$ в граничном условии $(5.12)$ в ряды Фурье по с.ф. $\varepsilon_{k}(x)$ оператора $S_{\mu}$ легко устанавливается следующее утверждение (см. [15], [16] для случая, соответствуюшего $\left.\Gamma_{2}=\varnothing\right)$.

Теорема 5.3. Система $\oint . \phi .\left\{E_{k}(x)\right\}_{k=1}^{\infty}$ Пуанкаре-Стеклова, соответствующая оператору $S_{\mu}$, ортонормирована относительно скалярного произве-

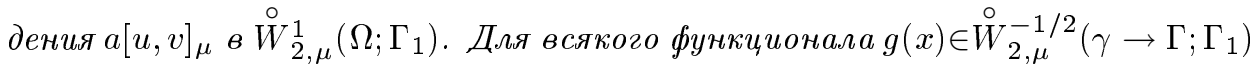
в (5.2) ряд

$$
\sum_{k=1}^{\infty} \nu_{k}^{-1 / 2} c_{k}(g) E_{k}(x),
$$

где $c_{k}(g)$ - коэффициенть Фурье функиионала $g(x)$ по системе с.ф. $\varepsilon_{k}(x)$ оператора $S_{\mu}$ (см. (5.19a)), сходится в $W_{2, \mu}^{1}(\Omega)$ к решению и $(x)$ задачи (5.1), (5.2). Для всякой функиии $\varphi(x) \in \stackrel{\circ}{W_{2, \mu}^{1 / 2}}\left(\gamma \rightarrow \Gamma ; \Gamma_{1}\right)$ в (5.12) ряд

$$
\sum_{k=1}^{\infty} \nu_{k}^{1 / 2} c_{k}(\varphi) E_{k}(x)
$$

где $c_{k}(\varphi)$ - коэффициенты Фурье функиии $\varphi(x)$ по системе с.ф. $\varepsilon_{k}(x)$ (см. $(5.19 б))$, сходится в $W_{2, \mu}^{1}(\Omega)$ к решению и $(x)$ задачи (5.1), (5.12).

ДокАЗАТЕЛЬСтво. Подставляя в (5.14) $g_{1}=\nu_{k}^{1 / 2} \varepsilon_{k}(x), g_{2}=\nu_{j}^{1 / 2} \varepsilon_{j}(x)$, имеем:

$$
\begin{aligned}
a\left[E_{k}, E_{j}\right]_{\mu} & =\left(\frac{\nu_{k}}{\nu_{j}}\right)^{1 / 2}\left\langle\varepsilon_{k}, \overline{\nu_{j} S_{\mu} \varepsilon_{j}}\right\rangle_{\gamma} \\
& =\left(\frac{\nu_{k}}{\nu_{j}}\right)^{1 / 2}\left\langle\varepsilon_{k}, \bar{\varepsilon}_{j}\right\rangle_{\gamma}=\left(\frac{\nu_{k}}{\nu_{j}}\right)^{1 / 2}\left(\varepsilon_{k}, \varepsilon_{j}\right)_{L_{2}(\gamma)}=\delta_{k}^{j} .
\end{aligned}
$$

Соотношения (5.14) и (5.15) с использованием (5.16a) и (5.16б) перепишем в виде

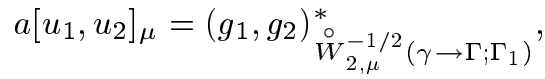

$$
\begin{aligned}
& a\left[u_{1}, u_{2}\right]_{\mu}=\left(\varphi_{1}, \varphi_{2}\right)_{W_{2, \mu}^{1 / 2}\left(\gamma \rightarrow \Gamma ; \Gamma_{1}\right)}^{*},
\end{aligned}
$$


соответственно. Если в $(5.2) g(x) \in \stackrel{\circ}{\stackrel{W}{W}_{2, \mu}^{-1 / 2}}\left(\gamma \rightarrow \Gamma ; \Gamma_{1}\right)$, то в силу теоремы 5.2 $g(x)-\sum_{k=1}^{N} c_{k}(g) \varepsilon_{k}(x) \rightarrow 0$ в $\stackrel{\circ}{W}-1 / 2\left(\gamma \rightarrow \Gamma ; \Gamma_{1}\right)$ при $N \rightarrow \infty$. Решением задачи $(5.1),(5.2)$ с $g(x)$, замененнып на $g(x)-\sum_{k=1}^{N} c_{k}(g) \varepsilon_{k}(x)$, согласно определению 5.3 будет функция $u(x)-\sum_{k=1}^{N} \nu_{k}^{-1 / 2} c_{k}(g) E_{k}(x)$, и в силу (5.21а) получаем, что

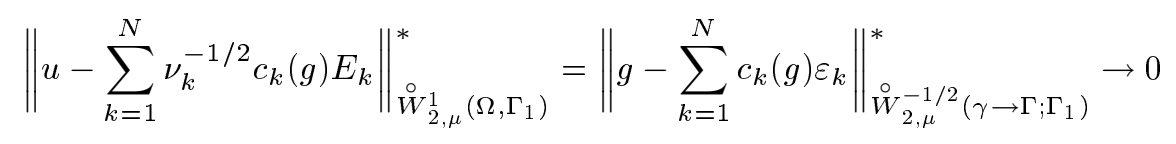

при $N \rightarrow \infty$, где

$$
\|u\|_{W_{2, \mu}^{1}\left(\Omega ; \Gamma_{1}\right)}^{*}=a[u, u]_{\mu}^{1 / 2}
$$

Совершенно аналогично с использованием (5.21б) устанавливается последнее утверждение теоремы 5.3.

В заключение параграфа следующее утверждение, которое будет использовано и в дальнейшем, выделим в виде леммы.

ЛЕмма 5.3. Если в дополнение к предъдущим предположениям $\Gamma_{2}$ - липшичев кусок границы $\Gamma$, то функиия $\varphi(x)$ принадлежит $\left.\stackrel{\circ}{{ }^{1 / 2}} \underset{2, \mu}{(\gamma} \rightarrow \Gamma ; \Gamma_{1}\right)$ тогда и только тогда, когда функиия

$$
\stackrel{\circ}{\varphi}(x)= \begin{cases}\varphi(x) & \text { nрu } x \in \gamma \\ 0 & \text { nрu } x \in \Gamma \backslash\left(\gamma \cup \bar{\Gamma}_{2}\right)\end{cases}
$$

принадлежст пространству $W_{2, \mu}^{1 / 2}\left(\Gamma \backslash \bar{\Gamma}_{2}\right)$. При этом

$$
\|\stackrel{\circ}{\varphi}\|_{W_{2, \mu}^{1 / 2}\left(\Gamma \backslash \bar{\Gamma}_{2}\right)} \leqslant\|\varphi\|_{W_{2, \mu}^{1 / 2}\left(\gamma \rightarrow \Gamma ; \Gamma_{1}\right)} \leqslant c\|\stackrel{\circ}{\varphi}\|_{W_{2, \mu}^{1 / 2}\left(\Gamma \backslash \bar{\Gamma}_{2}\right)}
$$

әде $c=c\left(\Gamma \backslash \bar{\Gamma}_{2} ; \Gamma\right)$ - постоянная, фигурирующая в $(2.12)$ (не зависит от $\left.\mu\right)$.

Доказательство аналогично доказательству утверждения, приведенного непосредственно перед теоремой 3.1 , и использует оператор продолжения $l_{\Lambda}^{\Gamma}$, существование которого гарантируется теоремой 2.1.2.

Таким образом, взаимно однозначное соответствие $\varphi \leftrightarrow \stackrel{\circ}{\varphi}$ устанавливает в этом случае отождествление пространств $\stackrel{\circ}{W} \underset{2, \mu}{1 / 2}\left(\gamma \rightarrow \Gamma ; \Gamma_{1}\right)$ и $\stackrel{\circ}{W}_{2, \mu}^{1 / 2}\left(\Gamma \backslash \bar{\Gamma}_{2} ; \Gamma_{1}\right)$. С этим получаем отождествление и двойственных пространств $\stackrel{\circ}{W_{2, \mu}^{-1 / 2}}\left(\gamma \rightarrow \Gamma ; \Gamma_{1}\right)$ и $\stackrel{\circ}{W}-1 / 2\left(\Gamma \backslash \bar{\Gamma}_{2} ; \Gamma_{1}\right)$. 


\section{§6. О смешанной задаче в цилиндрических областях. Обоснование метода Фурье}

Пусть $\Lambda$ - область с липшицевой границей $\partial \Lambda$ в $\mathbb{R}^{n-1}, \Omega=\Lambda \times\left(a_{0}, a_{1}\right), a_{0}<a_{1}$, - цилиндрическая область в $\mathbb{R}^{n}$. Пусть, далее, $\partial \Lambda$ разбита на два открытых липшицева куска $\gamma_{1}$ и $\gamma_{2}: \gamma_{1} \cap \gamma_{2}=\varnothing, \bar{\gamma}_{1} \cup \gamma_{2}=\partial \Lambda$ (в частном случае одно из $\gamma_{i}$ может быть пустым множеством). Обозначим: $\Lambda_{0}=\Lambda \times\left\{a_{0}\right\}$ и $\Lambda_{1}=\Lambda \times\left\{a_{1}\right\}-$ торцы цилиндра $\Omega, \Gamma_{1}=\gamma_{1} \times\left(a_{0}, a_{1}\right)$ и $\Gamma_{2}=\gamma_{2} \times\left(a_{0}, a_{1}\right)$ - части, составляющие $\Gamma_{0}=\partial \Lambda \times\left(a_{0}, a_{1}\right)-$ боковую поверхность цилиндра $\Omega$. Всю границу области $\Omega$ обозначаем, как и раньше, через $\Gamma$.

ЛЕмма 6.1. При сделанных предположениях $\Omega$ является тажже областью с липшицевой границей, $\Lambda_{0}, \Lambda_{1}, \Gamma_{0}, \Gamma_{1}$ и $\Gamma_{2}$ представляют собой липиицевь куски границь Г.

Доказательство этого интуитивно достаточно очевидного в $\mathbb{R}^{3}$ утверждения в общем случае технически довольно громоздко и будет опущено.

Настоящий параграф̆ в основном посвяшен обоснованию метода Фурье для смешанной задачи для однородного уравнения

$$
L(x, D, \mu) u(x)=0 \quad \text { в } \Omega
$$

при однородных граничных условиях на боковой поверхности

$$
\left.u(x)\right|_{\Gamma_{1}}=0,\left.\quad\left(\frac{\partial u}{\partial \nu}(x)+\sigma\left(x^{\prime}\right) u(x)\right)\right|_{\Gamma_{2}}=0
$$

и с неоднородными граничньми условиями на торцах одного из следующих видов:

$$
\begin{aligned}
\left.u(x)\right|_{\Lambda_{0}} & =\varphi_{0}(x), & \left.u(x)\right|_{\Lambda_{1}} & =\varphi_{1}(x) ; \\
\left.\frac{\partial u}{\partial \boldsymbol{n}}(x)\right|_{\Lambda_{0}} & =g_{0}(x), & & \left.\frac{\partial u}{\partial \boldsymbol{n}}(x)\right|_{\Lambda_{1}}=g_{1}(x) ; \\
\left.u(x)\right|_{\Lambda_{0}} & =\varphi_{0}(x), & & \left.\frac{\partial u}{\partial \boldsymbol{n}}(x)\right|_{\Lambda_{1}}=g_{1}(x)
\end{aligned}
$$

где $\partial / \partial \boldsymbol{n}$ - производная по направлению внешней нормали. Оператор (6.1) предполагаем допускающим разделение переменных на группу $x^{\prime}=\left(x_{1}, \ldots, x_{n-1}\right)$ и переменную $x_{n}$ вида

$$
L(x, D, \mu)=L^{\prime}\left(x^{\prime}, D^{\prime}\right)-D_{n}^{2}+\mu^{2} I
$$

где

$$
L^{\prime}\left(x^{\prime}, D^{\prime}\right) u(x)=-\sum_{i, j=1}^{n-1} D_{i}\left(a_{i j}\left(x^{\prime}\right) D_{j} u(x)\right)+a_{0}\left(x^{\prime}\right) u(x)
$$


$a_{i j}\left(x^{\prime}\right) \in L_{\infty}(\Lambda)$ и образуют эрмитову равномерно по $x^{\prime} \in \Lambda$ положительно определенную матрицу:

$$
\sum_{i, j=1}^{n-1} a_{i j}\left(x^{\prime}\right) \zeta_{i} \bar{\zeta}_{j} \geqslant \alpha_{0}\left|\zeta^{\prime}\right|^{2} \quad \forall \zeta^{\prime} \in \mathbb{C}^{n-1}, \quad \alpha_{0}=\text { const }>0
$$

Предполагается также, что $a_{0}\left(x^{\prime}\right) \in L_{\infty}(\Lambda), \sigma\left(x^{\prime}\right) \in L_{\infty}\left(\gamma_{2}\right)$ - действительные функции, и что $\mu \geqslant \mu_{0}>0$, где $\mu_{0}$ - столь большое, чтобы рассматриваемые задачи были однозначно разрешимы.

Сначала получим такие формулировки обобшенных решений рассматриваемых задач, в которых функции и функционалы правых частей неоднородных граничных условий (6.3а)-(6.3в) представляют собой соответствующие объекты уже только на одном и том же поперечном сечении $\Lambda$ цилиндра $\Omega$.

Исходя из рассмотрений $\S 3$ и $\S 5$, задачу $(6.1),(6.2),(6.3 \mathrm{a})$ будем понимать как задачу вида (5.1), (5.12) с $\gamma=\Lambda_{0} \cup \Lambda_{1}$ и с

$$
\varphi(x)= \begin{cases}\varphi_{0}(x) & \text { при } x \in \Lambda_{0} \\ \varphi_{1}(x) & \text { при } x \in \Lambda_{1}\end{cases}
$$

При этом следует полагать, что

$$
\varphi(x) \in \stackrel{\circ}{W}{ }_{2, \mu}^{1 / 2}\left(\Lambda_{0} \cup \Lambda_{1} \rightarrow \Gamma ; \Gamma_{1}\right) .
$$

При достаточно больших $\mu, \mu \geqslant \mu_{0}$, задача имеет единственное обобщенное решение $u(x)$, для которого выполняется оценка вида (3.11) с $f$ и $g$ нулевыми и с членом $\|\varphi\|_{W_{2,|\mu|}^{1 / 2}\left(\Gamma_{1} \rightarrow \Gamma\right)}$, замененным на $\|\varphi\|_{W_{2, \mu}^{\circ / 2}\left(\Lambda_{0} \cup \Lambda_{1} \rightarrow \Gamma ; \Gamma_{1}\right)}$.

Задачу $(6.1),(6.2),(6.3 б)$ в силу соответствующих рассмотрений $\S 5$ будем понимать как задачу вида (5.1), (5.2), опять же с $\gamma=\Lambda_{0} \cup \Lambda_{1}$ и с

$$
g(x)= \begin{cases}g_{0}(x) & \text { на } \Lambda_{0} \\ g_{1}(x) & \text { на } \Lambda_{1}\end{cases}
$$

(однако здесь требуется еще придать смысл последнему выражению, что будет сделано ниже). Должно выполняться включение

$$
g(x) \in \stackrel{\circ}{W}-1 / 2, \mu\left(\Lambda_{0} \cup \Lambda_{1} \rightarrow \Gamma ; \Gamma_{1}\right) .
$$

Такую задачу на самом деле нужно понимать как задачу вида (5.1), (5.3) с функционалом $\stackrel{\circ}{g}(x) \in \stackrel{\circ}{W} \underset{2, \mu}{-1 / 2}\left(\Gamma \backslash \bar{\Gamma}_{1}\right)$, представляющим собой “продолжение нулем на $\Gamma_{2}$ " функционала $g(x)$ в смысле леммы 5.1. При достаточно больших $\mu, \mu \geqslant \mu_{0}$, задача имеет единственное обобшенное решение $u(x)$, для которого выполняется оценка вида самого правого неравенства в (5.11). 
Задачу (6.1), (6.2), (6.3в) будем рассматривать как обобщение задачи (5.1), (5.2) с $\gamma=\Lambda_{1}, g(x)=g_{1}(x)$, для которого однородное граничное условие $\left.u\right|_{\Gamma_{1}}=0$ заменено на неоднородное: $\left.u\right|_{\Gamma_{1}}=0,\left.u\right|_{\Lambda_{0}}=\varphi_{0}$. Итак, в (5.1), (5.2) $\Gamma_{1}$ заменяется на $\widetilde{\Gamma}_{1}=\operatorname{int}\left(\overline{\Gamma_{1} \cup \Lambda_{0}}\right)$ и на этом множестве задается функция

$$
\varphi(x)= \begin{cases}\varphi_{0}(x) & \text { при } x \in \Lambda_{0}, \\ 0 & \text { при } x \in \widetilde{\Gamma}_{1} \backslash \Lambda_{0} .\end{cases}
$$

Предполагаем, что

$$
\begin{aligned}
& g_{1}(x) \in \stackrel{\circ}{W_{2, \mu}^{-1 / 2}}\left(\Lambda_{1} \rightarrow \Gamma ; \Gamma_{1} \cup \Lambda_{0}\right), \\
& \varphi_{0}(x) \in \stackrel{\circ}{{ }^{1 / 2}} \underset{2, \mu}{1 / 2}\left(\Lambda_{0} \rightarrow \Gamma ; \Gamma_{1}\right) .
\end{aligned}
$$

Отметим, что последнее условие эквивалентно условию $\varphi(x) \in W_{2, \mu}^{1 / 2}\left(\widetilde{\Gamma}_{1} \rightarrow \Gamma\right)$.

При выполнении первого условия (6.6в) по функционалу $g_{1}(x)$ согласно лемме 5.1 может быть построен функционал $\stackrel{\circ}{g}(x) \in \stackrel{\circ}{W_{2, \mu}^{-1 / 2}}\left(\widetilde{\Gamma}_{2}\right)$, где $\widetilde{\Gamma}_{2}=\operatorname{int}\left(\overline{\Gamma_{2} \cup \Lambda_{1}}\right)$, являюшийся "продолжением нулем на $\Gamma_{2}$ " функционала $g_{1}(x)$, и задача $(6.1),(6.2)$, (6.3в) понимается как задача вида $(1.1),(1.2)$ :

$$
L(x, D, \mu) u=0 \quad \text { в } \quad \Omega,\left.\quad u\right|_{\widetilde{\Gamma}_{1}}=\varphi,\left.\quad\left(\frac{\partial u}{\partial \nu}+\stackrel{\circ}{\sigma} u\right)\right|_{\widetilde{\Gamma}_{2}}=\stackrel{\circ}{g}
$$

где

$$
\stackrel{\circ}{\sigma}(x)= \begin{cases}\sigma\left(x^{\prime}\right) & \text { при } x \in \Gamma_{2}, \\ 0 & \text { при } x \in \Lambda_{1} .\end{cases}
$$

Обобщенным решением такой задачи является функция $u(x) \in \stackrel{\circ}{W} \underset{2, \mu}{1}\left(\Omega ; \Gamma_{1}\right)$, удовлетворяюшая граничному условию

$$
\left.u(x)\right|_{\Lambda_{0}}=\varphi_{0}(x)
$$

и $\forall v(x) \in \stackrel{\circ}{W} \frac{1}{2, \mu}\left(\Omega ; \Gamma_{1} \cup \Lambda_{0}\right)$ - соотношению

$$
a[u, v]_{\mu}=\left\langle g_{1}, \bar{v}\right\rangle_{\Lambda_{1}},
$$

где

$$
\begin{aligned}
a[u, v]_{\mu}= & \int_{a_{0}}^{a_{1}} a^{\prime}[u, v]_{\mu} d x_{n}+\int_{\Omega} D_{n} u D_{n} \bar{v} d x \\
a^{\prime}[u, v]_{\mu}= & \int_{\Lambda}\left[\sum_{i, j=1}^{n-1} a_{i j}\left(x^{\prime}\right) D_{j} u(x) D_{i} \bar{v}(x)+\left(a_{0}\left(x^{\prime}\right)+\mu^{2}\right) u(x) \bar{v}(x)\right] d x^{\prime} \\
& +\int_{\gamma_{1}} \sigma\left(x^{\prime}\right) u(x) \bar{v}(x) d s_{x^{\prime}} .
\end{aligned}
$$

Последняя задача имеет единственное решение $u(x)$ при $\mu \geqslant \mu_{0}$, где $\mu_{0}$ достаточно большое, и при этом выполняется оценка вида (3.11):

$$
\|u\|_{W_{2, \mu}^{1}(\Omega)} \leqslant\left(c_{1}\left\|\varphi_{0}\right\|_{\stackrel{\circ}{2, \mu}_{2}^{1 / 2}\left(\Lambda_{0} \rightarrow \Gamma ; \Gamma_{1}\right)}+c_{2}\left\|g_{1}\right\|_{\stackrel{\circ}{2, \mu}_{2}^{-1 / 2}\left(\Lambda_{1} \rightarrow \Gamma ; \Gamma_{1} \cup \Lambda_{0}\right)}\right) .
$$

Ниже под эквивалентностью двух зависяших от параметра $\mu$ нормировок будем понимать, не оговаривая это, наличие двусторонних оценок с оценивающими постоянными, не зависящими от $\mu$. 
Лемма 6.2. 1. Пространства $\stackrel{\circ}{\mathrm{W}} \underset{2, \mu}{1 / 2}\left(\Lambda_{m} \rightarrow \Gamma ; \Gamma_{1}\right)$ u $\stackrel{\circ}{{ }^{1 / 2}} \underset{2, \mu}{ }\left(\Lambda_{m} \rightarrow \Gamma ; \Gamma_{1} \cup \Lambda_{1-m}\right)$ совпадают; их нормы әквивалентны, $m=0,1$.

2. Включение (6.6а) для функиии (6.5a) имеет место тогда и только тогда, когда $\varphi_{m}(x) \in \stackrel{\circ}{W_{2, \mu}^{1 / 2}}\left(\Lambda_{m} \rightarrow \Gamma ; \Gamma_{1}\right), m=0,1$. Норма функиии (6.5а) в

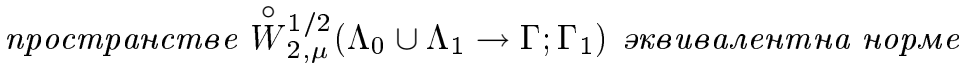

$$
\sum_{m=0}^{1}\left\|\varphi_{m}\right\|_{\stackrel{\circ}{W}_{2, \mu}^{1 / 2}\left(\Lambda_{m} \rightarrow \Gamma ; \Gamma_{1}\right)} .
$$

ДокАЗАТЕЛЬство. 1. Из определений непосредственно следует оценка

$$
\left\|\varphi_{m}\right\|_{\stackrel{\circ}{W}_{2, \mu}^{1 / 2}\left(\Lambda_{m} \rightarrow \Gamma ; \Gamma_{1}\right)} \leqslant\left\|\varphi_{m}\right\|_{\stackrel{\circ}{W}_{2, \mu}^{1 / 2}\left(\Lambda_{m} \rightarrow \Gamma ; \Gamma_{1} \cup \Lambda_{1-m}\right)} .
$$

Обозначим, далее, $\eta_{0}(x)=\left(a_{1}-x_{n}\right) /\left(a_{1}-a_{0}\right), \eta_{1}(x)=\left(x_{n}-a_{0}\right) /\left(a_{1}-a_{0}\right)$. Тогда, если $l \varphi_{m} \in \stackrel{\circ}{W_{2, \mu}^{1 / 2}}\left(\Gamma ; \Gamma_{1}\right)$ такая, что $\left.l \varphi_{m}\right|_{\Lambda_{m}}=\varphi_{m}$ и

$$
\left\|l \varphi_{m}\right\|_{W_{2, \mu}^{1 / 2}(\Gamma)} \leqslant 2\left\|\varphi_{m}\right\|_{W_{2, \mu}^{1 / 2}\left(\Lambda_{m} \rightarrow \Gamma ; \Gamma_{1}\right)},
$$

то в силу леммы $2.1 \eta_{m}(x) l \varphi_{m}(x) \in \stackrel{\circ}{W_{2, \mu}^{1 / 2}}\left(\Lambda_{m} \rightarrow \Gamma ; \Gamma_{1} \cup \Lambda_{1-m}\right)$ и

$$
\left\|\varphi_{m}\right\|_{\stackrel{\circ}{W}_{2, \mu}^{1 / 2}\left(\Lambda_{m} \rightarrow \Gamma ; \Gamma_{1} \cup \Lambda_{1-m}\right)} \leqslant\left\|\eta_{m} l \varphi_{m}\right\|_{W_{2, \mu}^{1 / 2}(\Gamma)} \leqslant c\|\varphi\|_{\stackrel{\circ}{2, \mu}_{2 / 2}^{1 / 2}\left(\Lambda_{m} \rightarrow \Gamma ; \Gamma_{1}\right)},
$$

где $c$ - постоянная, не зависяшая от $\mu$. Итак, первое утверждение леммы установлено.

2. На основании определений функции (6.5a), удовлетворяющей (6.6а), и встречающихся пространств легко получаем, что

$$
\begin{aligned}
\left\|\varphi_{k}\right\|_{\stackrel{\circ}{W}_{2, \mu}^{1 / 2}\left(\Lambda_{k} \rightarrow \Gamma ; \Gamma_{1}\right)} & \leqslant\|\varphi\|_{\stackrel{\circ}{W}_{2, \mu}^{1 / 2}\left(\Lambda_{0} \cup \Lambda_{1} \rightarrow \Gamma ; \Gamma_{1}\right)} \\
& \leqslant \sum_{m=0}^{1}\left\|\varphi_{m}\right\|_{\stackrel{\circ}{W}_{2, \mu}^{1 / 2}\left(\Lambda_{m} \rightarrow \Gamma ; \Gamma_{1} \cup \Lambda_{1-m}\right)}, \quad k=0,1 .
\end{aligned}
$$

Эта оценка с учетом уже доказанного устанавливает справедливость второй части леммы 6.2 .

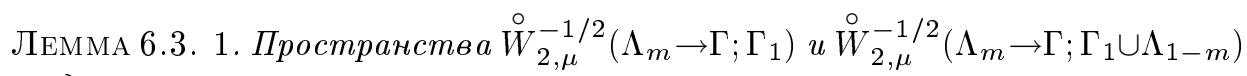
совпадают, а их нормы әквивалентныл.

2. Для всякого функиионала $g(x)$, удовлетворяющего (6.6б), существует однозначно определенные функичональ $g_{m}(x) \in \stackrel{\circ}{W_{2, \mu}^{-1 / 2}}\left(\Lambda_{m} \rightarrow \Gamma ; \Gamma_{1}\right), m=0,1$, такие, что для любой функичи (6.5a), удовлетворяющей (6.6a),

$$
\langle g, \varphi\rangle_{\Lambda_{0} \cup \Lambda_{1}}=\sum_{m=0}^{1}\left\langle g_{m},\left.\varphi\right|_{\Lambda_{m}}\right\rangle_{\Lambda_{m}} .
$$


Обратно, для любъхх двух функционалов $g_{m}(x) \in \stackrel{\circ}{W} \underset{2, \mu}{-1 / 2}\left(\Lambda_{m} \rightarrow \Gamma ; \Gamma_{1}\right), m=0,1$, функционал $g(x)$, определяемый по формуле (6.14), удовлетворяет (6.6б), и при этом

$$
\|g\|_{\stackrel{\circ}{W}_{2, \mu}^{-1 / 2}\left(\Lambda_{0} \cup \Lambda_{1} \rightarrow \Gamma ; \Gamma_{1}\right)} \leqslant \sum_{m=0}^{1}\left\|g_{m}\right\|_{\stackrel{\circ}{2, \mu}_{2 / 2}^{1 / 2}\left(\Lambda_{m} \rightarrow \Gamma ; \Gamma_{1}\right)} .
$$

ДокАЗАТЕЛЬСтво. Первое утверждение непосредственно вытекает из п. 1 леммы 6.2 .

2. Для каждого $m=0,1$ определим линейньй оператор $\lambda_{m}$, которьй всякую функцию $\varphi_{m}(x) \in \stackrel{\circ}{{ }^{1}} \underset{2, \mu}{2 / 2}\left(\Lambda_{m} \rightarrow \Gamma ; \Gamma_{1}\right)$ переводит в функцию

$$
\lambda_{m} \varphi_{m}(x)= \begin{cases}\varphi_{m}(x) & \text { при } x \in \Lambda_{m}, \\ 0 & \text { при } x \in \Lambda_{1-m} .\end{cases}
$$

Поскольку

$$
\begin{aligned}
\left\|\lambda_{m} \varphi_{m}\right\|_{\stackrel{\circ}{2, \mu}_{2}^{1 / 2}\left(\Lambda_{0} \cup \Lambda_{1} \rightarrow \Gamma ; \Gamma_{1}\right)} & \left.=\left\|\varphi_{m}\right\|_{\stackrel{\circ}{2, \mu}_{2 / 2}^{1 / 2}\left(\Lambda_{m} \rightarrow \Gamma ; \Gamma\right.} \cup \Lambda_{1-m}\right) \\
& \leqslant c\left\|\varphi_{m}\right\|_{W_{2, \mu}^{1 / 2}\left(\Lambda_{m} \rightarrow \Gamma ; \Gamma_{1}\right)},
\end{aligned}
$$

то непрерывен каждьй из операторов $\lambda_{m}$. Поэтому функционалы $g_{m}$, определяемые по формуле

$$
\left\langle g_{m}, \varphi_{m}\right\rangle_{\Lambda_{m}}=\left\langle g, \lambda_{m} \varphi_{m}\right\rangle_{\Lambda_{0} \cup \Lambda_{1}}
$$

принадлежат пространствам $\stackrel{\circ}{W} \underset{2, \mu}{-1 / 2}\left(\Lambda_{m} \rightarrow \Gamma ; \Gamma_{1}\right)$. Всякую функцию (6.5а), удовлетворяюшую (6.6а), представим

$$
\varphi=\left.\sum_{m=0}^{1} \lambda_{m} \varphi\right|_{\Lambda_{m}} .
$$

Подставляя это разложение в $\langle g, \varphi\rangle_{\Lambda_{0} \cup \Lambda_{1}}$, приходим к представлению (6.14) с функционалами $g_{m}$, определяемыми по формуле (6.16).

Единственность представления (6.14) устанавливается при подстановке в него $\varphi=\left.\lambda_{k} \varphi\right|_{\Lambda_{k}}$ : для $g_{m}$ тогда необходимо получаются выражения (6.16).

Наконец, если $g_{m}(x) \in \stackrel{\circ}{W_{2, \mu}^{-1 / 2}}\left(\Lambda_{m} \rightarrow \Gamma ; \Gamma_{1}\right), m=0,1,-$ произвольнье, то формула (6.14) определяет функционал $g(x) \in \stackrel{\circ}{W_{2, \mu}^{-1 / 2}}\left(\Lambda_{0} \cup \Lambda_{1} \rightarrow \Gamma ; \Gamma_{1}\right)$, причем, в силу левой части оценки (6.13), получаем (6.15).

Отметим, что в доказательствах лемм 6.2 и 6.3 не использовалось то условие, что $\gamma_{1}-$ липшицев кусок $\partial \Lambda$.

Перейдем теперь к трактовке встречающихся функций и функционалов на торцах $\Lambda_{0}$ и $\Lambda_{1}$ как соответствуюших объектов на сечении $\Lambda \subset \mathbb{R}^{n-1}$. Для функций это тривиально. Всякой функции $\varphi_{m}(x)$, определенной на $\Lambda_{m}$, поставим во взаимно однозначное соответствие функцию

$$
\varphi_{m}\left(x^{\prime}, a_{m}\right), \quad x^{\prime} \in \Lambda,
$$


определенную на $\Lambda$. Покажем, что все встречающиеся выше пространства функций на $\Lambda_{m}$ очень просто описываются принадлежностью функции (6.17) “эталонному" пространству $\stackrel{\circ}{\underset{W}{2}} \underset{2, \mu}{(2}\left(\Lambda ; \gamma_{1}\right)$, которое сейчас определим.

Обозначим в $\mathbb{R}^{n}$ через $\stackrel{\circ}{\Lambda}$ и $\Sigma_{1}^{ \pm}$следуюшие множества:

$$
\stackrel{\circ}{\Lambda}=\Lambda \times\{0\}, \quad \Sigma_{1}^{+}=\gamma_{1} \times[0,1), \quad \Sigma_{1}^{-}=\gamma_{1} \times(-1,0] .
$$

ОПредЕлениЕ 6.1. Скажем, что функция $\varphi\left(x^{\prime}\right)$ из $W_{2}^{1 / 2}(\Lambda)$ принадлежит пространству $\stackrel{\circ}{W}{ }_{2, \mu}^{1 / 2}\left(\Lambda ; \gamma_{1}\right)$, если одна из функций

$$
\stackrel{\circ}{\varphi}^{ \pm}(x)= \begin{cases}\varphi\left(x^{\prime}\right) & \text { при }\left(x^{\prime}, x_{n}\right) \in \stackrel{\circ}{\Lambda}, \\ 0 & \text { при } x \in \Sigma_{1}^{ \pm},\end{cases}
$$

например, $\stackrel{\circ}{\varphi}^{+}(x)$, принадлежит пространству $W_{2, \mu}^{1 / 2}\left(\stackrel{\circ}{\Lambda} \cup \Sigma_{1}^{+}\right.$) (тогда и другая функция $\stackrel{\circ}{\varphi}^{-}(x)$ - принадлежит $\left.W_{2, \mu}^{1 / 2}\left(\stackrel{\circ}{\Lambda} \cup \Sigma_{1}^{-}\right)\right)$. Норму в $\stackrel{\circ}{W} \underset{2, \mu}{1 / 2}\left(\Lambda ; \gamma_{1}\right)$ определим любым из выражений:

$$
\|\varphi\|_{W_{2, \mu}^{1 / 2}\left(\Lambda ; \gamma_{1}\right)}=\left\|\stackrel{\circ}{\varphi}^{+}\right\|_{W_{2, \mu}^{1 / 2}\left(\stackrel{\circ}{\Lambda} \cup \Sigma_{1}^{+}\right)}=\left\|\stackrel{\circ}{\varphi}^{-}\right\|_{W_{2, \mu}^{1 / 2}\left(\stackrel{\circ}{\Lambda} \cup \Sigma_{1}^{-}\right)} .
$$

Лемма 6.4. Пусть выполнень условия, приведенные в начале §6. Тогда функция $\varphi_{m}(x), m=0$ или 1 , принадлежит пространству $\stackrel{\circ}{{ }_{2}^{1 / 2}}\left(\Lambda_{m} \rightarrow \Gamma ; \Gamma_{1}\right)$ $\left(=\stackrel{\circ}{W} \underset{2, \mu}{1 / 2}\left(\Lambda_{m} \rightarrow \Gamma ; \Gamma_{1} \cup \Lambda_{1-m}\right)\right)$ тогда и только тогда, когда функция (6.17) принадлежит пространству $\stackrel{\circ}{W_{2, \mu}^{1 / 2}}\left(\Lambda ; \gamma_{1}\right)$. Норма $\varphi_{m}(x)$ в $\stackrel{\circ}{W_{2, \mu}^{1 / 2}}\left(\Lambda_{m} \rightarrow \Gamma ; \Gamma_{1}\right)$ $\left(\right.$ или в $\left.\stackrel{\circ}{W} \underset{2, \mu}{1 / 2}\left(\Lambda_{m} \rightarrow \Gamma ; \Gamma_{1} \cup \Lambda_{1-m}\right)\right)$ әквивалентна норме функиии $\varphi_{m}\left(x^{\prime}, a_{m}\right)$ в $\stackrel{\circ}{W}{ }_{2, \mu}^{1 / 2}\left(\Lambda ; \gamma_{1}\right)$.

ДокАЗАТЕЛЬСТво. Установим это утверждение, например, для $\varphi_{1}(x)$. Без ограничения обшности при этом можем предполагать, что $a_{1}=0$, а потому $\stackrel{\circ}{\Lambda}=\Lambda_{1}$. Обозначим через

$$
\stackrel{\circ}{\varphi}_{1}(x)= \begin{cases}\varphi_{1}(x) & \text { при } x \in \Lambda_{1}, \\ 0 & \text { при } x \in \Gamma_{1}\end{cases}
$$

а через

$$
\stackrel{\circ}{\varphi}_{1}^{-}(x)= \begin{cases}\varphi_{1}(x) & \text { при } x \in \Lambda_{1}=\stackrel{\circ}{\Lambda}, \\ 0 & \text { при } x \in \Sigma_{1}^{-} .\end{cases}
$$

В силу леммы 5.3 , поскольку согласно лемме 6.1 множество int $\left(\overline{\Gamma_{2} \cup \Lambda_{0}}\right)$ является липшицевьм куском $\Gamma$, можем оценить

$$
\left\|\stackrel{\circ}{\varphi}_{1}\right\|_{W_{2, \mu}^{1 / 2}\left(\Lambda_{1} \cup \Gamma_{1}\right)} \leqslant\left\|\varphi_{1}\right\|_{\stackrel{\circ}{2, \mu}_{2,2}^{1 / 2}\left(\Lambda_{1} \rightarrow \Gamma ; \Gamma_{1}\right)} \leqslant c\left\|\stackrel{\circ}{\varphi}_{1}\right\|_{W_{2, \mu}^{1 / 2}\left(\Lambda_{1} \cup \Gamma_{1}\right)} .
$$


В случае, когда $\left(a_{1}-a_{0}\right)=1$, эта оценка и является уже доказываемой оценкой, поскольку $\bar{\Gamma}_{1}=\bar{\Sigma}_{1}^{-}$и $\stackrel{\circ}{\varphi}_{1}(x)=\stackrel{\circ}{\varphi}_{1}^{-}(x)$.

Пусть теперь $\left(a_{1}-a_{0}\right)>1$. Тогда $\Sigma_{1}^{-} \subset \bar{\Gamma}_{1}$. Обозначим $\Delta^{-}=\Gamma_{1} \backslash \Sigma_{1}^{-}$. Тогда $\operatorname{dist}\left\{\Lambda_{1}, \Delta^{-}\right\}=1$, а потому можем оценить

$$
\begin{aligned}
& \left\|\stackrel{\circ}{\varphi}_{1}^{-}\right\|_{W_{2, \mu}^{1 / 2}\left(\stackrel{\circ}{\Lambda} \cup \Sigma_{1}^{-}\right)}^{2} \leqslant\left\|\stackrel{\circ}{\varphi}_{1}\right\|_{W_{2, \mu}^{1 / 2}\left(\Lambda_{1} \cup \Gamma_{1}\right)}^{2}
\end{aligned}
$$

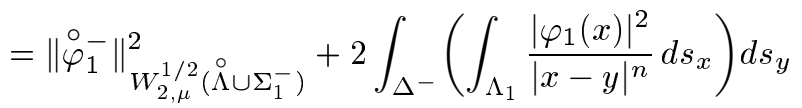

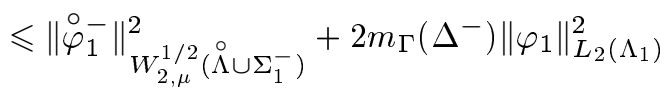

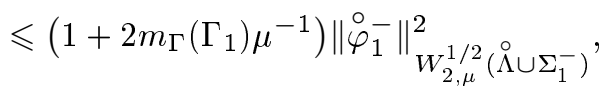

что вместе с (6.19) дает желаемую оценку эквивалентности.

Случай $\left(a_{1}-a_{0}\right)<1$ обрабатьвается аналогично: теперь $\Gamma_{1}$ и $\Sigma_{1}^{-}$как бы меняются ролями.

Как следствие этой леммы и леммы 6.2.2 получаем, что для функции (6.5a) включение (6.6а) имеет место тогда и только тогда, когда функции $\varphi_{m}\left(x^{\prime}, a_{m}\right)$, $m=0,1$, принадлежат пространству $\stackrel{\circ}{W_{2, \mu}^{1 / 2}}\left(\Lambda ; \gamma_{1}\right)$. При этом норма $(6,6 \mathrm{a})$ эквивалентна норме

$$
\sum_{m=0}^{1}\left\|\varphi_{m}\left(x^{\prime}, a_{m}\right)\right\|_{\stackrel{\circ}{2, \mu}_{2,2}^{1 / 2}\left(\Lambda ; \gamma_{1}\right)} .
$$

Перейдем теперь к функционалам. По функционалам $g_{m}(x)$ на $\Lambda_{m}$ построим функционалы $g_{m}\left(x^{\prime}, a_{m}\right)$ на $\Lambda$.

ОПРЕДЕЛЕНИЕ 6.2. Пусть $g_{m}(x), m=0$ или $1,-$ линейньй функционал, действуюший на некотором линейном пространстве $\mathscr{L}_{m}\left(\Lambda_{m}\right)$ функций, определенных на $\Lambda_{m}$. Совокупность функций (6.17), определенных на $\Lambda$, где $\varphi_{m}$ пробегают все $\mathscr{L}_{m}\left(\Lambda_{m}\right)$, образуют линейное пространство, которое обозначим через $\mathscr{L}_{m}(\Lambda)$. Тогда для любой $\varphi\left(x^{\prime}\right) \in \mathscr{L}_{m}(\Lambda)$ функция $\varphi_{m}(x)$ на $\Lambda_{m}$ такая, что $\varphi_{m}\left(x^{\prime}, a_{m}\right)=$ $\varphi\left(x^{\prime}\right)$, принадлежит $\mathscr{L}_{m}\left(\Lambda_{m}\right)$. Обозначим через $g_{m}\left(x^{\prime}, a_{m}\right)$ линейньй функционал на $\mathscr{L}_{m}(\Lambda)$, действующий на $\varphi\left(x^{\prime}\right)$ по формуле

$$
\left\langle g_{m}\left(x^{\prime}, a_{m}\right), \varphi\left(x^{\prime}\right)\right\rangle_{\Lambda}=\left\langle g_{m}(x), \varphi_{m}(x)\right\rangle_{\Lambda_{m}}
$$

Непосредственным следствием этого определения и предыдущих лемм являются следуюшие утверждения.

Лемма 6.5. 1. Функиионал $g_{m}(x), m=0$ или 1 , принадлежит пространству $\stackrel{\circ}{W} \underset{2, \mu}{-1 / 2}\left(\Lambda_{m} \rightarrow \Gamma ; \Gamma_{1}\right)\left(=\stackrel{\circ}{W_{2, \mu}^{-1 / 2}}\left(\Lambda_{m} \rightarrow \Gamma ; \Gamma_{1} \cup \Lambda_{1-m}\right)\right)$ тогда и только тогда, когда $g_{m}\left(x^{\prime}, a_{m}\right) \in \stackrel{\circ}{W_{2, \mu}^{-1 / 2}}\left(\Lambda ; \gamma_{1}\right)$. Норма $g_{m}(x)$ в $\stackrel{\circ}{\stackrel{\circ}{W}_{2, \mu}^{-1 / 2}}\left(\Lambda_{m} \rightarrow \Gamma ; \Gamma_{1}\right)$ 
$\left(\right.$ или в $\left.\stackrel{\circ}{W}{ }_{2, \mu}^{-1 / 2}\left(\Lambda_{m} \rightarrow \Gamma ; \Gamma_{1} \cup \Lambda_{1-m}\right)\right)$ әквивалентна норме $g_{m}\left(x^{\prime}, a_{m}\right)$ в пространстве $\stackrel{\circ}{W} \underset{2, \mu}{-1 / 2}\left(\Lambda ; \gamma_{1}\right)$. Для любой $\varphi_{m}(x) \in \stackrel{\circ}{{ }^{1 / 2}} \underset{2, \mu}{ }\left(\Lambda_{m} \rightarrow \Gamma ; \Gamma_{1}\right)$

$$
\left\langle g_{m}(x), \varphi_{m}(x)\right\rangle_{\Lambda_{m}}=\left\langle g_{m}\left(x^{\prime}, a_{m}\right), \varphi_{m}\left(x^{\prime}, a_{m}\right)\right\rangle_{\Lambda} .
$$

2. Eсли $g(x)$ - функционал, удовлетворяющий (6.6б), $g_{m}(x)$ - компоненты әтого функционала на $\Lambda_{m}$ в смысле $n .2$ леммы 6.3, то $\forall \varphi(x)$, удовлетворяющей (6.6a),

$$
\langle g, \varphi\rangle_{\Lambda}=\sum_{m=0}^{1}\left\langle g_{m}\left(x^{\prime}, a_{m}\right), \varphi_{m}\left(x^{\prime}, a_{m}\right)\right\rangle_{\Lambda} .
$$

На основании введенных понятий переформулируем рассматриваемые задачи в следуюшей форме.

Формально задачи (6.1), (6.2), (6.3а)-(6.3в) будем записьвать, соответственно, как задачи для уравнения (6.1), при однородных граничных условиях (6.2) и с неоднородными граничными условиями на торцах вида

$$
\begin{aligned}
u\left(x^{\prime}, a_{0}\right) & =\varphi_{0}\left(x^{\prime}\right), & u\left(x^{\prime}, a_{1}\right) & =\varphi_{1}\left(x^{\prime}\right) ; \\
\frac{\partial u}{\partial \boldsymbol{n}}\left(x^{\prime}, a_{0}\right) & =g_{0}\left(x^{\prime}\right), & \frac{\partial u}{\partial \boldsymbol{n}}\left(x^{\prime}, a_{1}\right) & =g_{1}\left(x^{\prime}\right) ; \\
u\left(x^{\prime}, a_{0}\right) & =\varphi_{0}\left(x^{\prime}\right), & \frac{\partial u}{\partial \boldsymbol{n}}\left(x^{\prime}, a_{1}\right) & =g_{1}\left(x^{\prime}\right),
\end{aligned}
$$

где через $\varphi_{m}\left(x^{\prime}\right)$ и $g_{m}\left(x^{\prime}\right)$ для сокращения обозначены, соответственно, $\varphi_{m}\left(x^{\prime}, a_{m}\right)$ и $g_{m}\left(x^{\prime}, a_{m}\right), m=0,1$. Предполагаем, что

$$
\varphi_{0}\left(x^{\prime}\right), \varphi_{1}\left(x^{\prime}\right) \in \stackrel{\circ}{{ }^{1}}{ }_{2, \mu}^{1 / 2}\left(\Lambda ; \gamma_{1}\right), \quad g_{0}\left(x^{\prime}\right), g_{1}\left(x^{\prime}\right) \in \stackrel{\circ}{{ }^{-1 / 2}}-2\left(\Lambda ; \gamma_{1}\right) .
$$

О.p. задачи $(6.1),(6.2),(6.22 \mathrm{a})$ является функция $u(x) \in \stackrel{\circ}{W} \underset{2, \mu}{1}\left(\Omega ; \Gamma_{1}\right)$, удовле-

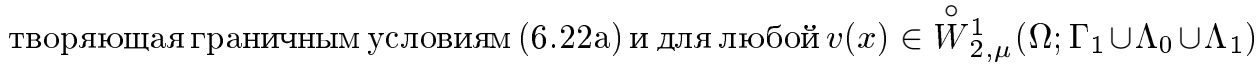
- соотношению

$$
a[u, v]_{\mu}=0 .
$$

О.p. задачи $(6.1),(6.2),(6.22 б)$ является функция $u(x) \in \stackrel{\circ}{W} \underset{2, \mu}{1}\left(\Omega ; \Gamma_{1}\right)$, удовлетворяющая для любой $v(x) \in \stackrel{\circ}{W} \underset{2, \mu}{1}\left(\Omega ; \Gamma_{1}\right)$ соотношению

$$
a[u, v]_{\mu}=\sum_{m=0}^{1}\left\langle g_{m}\left(x^{\prime}\right), \overline{v\left(x^{\prime}, a_{m}\right)}\right\rangle_{\Lambda} .
$$

О.p. задачи $(6.1),(6.2),(6.22$ в $)$ является функция $u(x) \in \stackrel{\circ}{W} \underset{2, \mu}{1}\left(\Omega ; \Gamma_{1}\right)$, удовлетворяющая первому граничному условию в (6.22в) и для любой функции $v(x) \in$ $\stackrel{\circ}{W} \underset{2, \mu}{1}\left(\Omega ; \Gamma_{1} \cup \Lambda_{0}\right)$ - соотношению

$$
a[u, v]_{\mu}=\left\langle g_{1}\left(x^{\prime}\right), \overline{v\left(x^{\prime}, a_{1}\right)}\right\rangle_{\Lambda}
$$


При $\mu \geqslant \mu_{0}$, где $\mu_{0}$ - достаточно большое, для обобшенного решения $u(x)$ каждой из этих задач имеет место, соответственно, оценка

$$
\begin{aligned}
& \|u\|_{W_{2, \mu}^{1}(\Omega)} \leqslant c \sum_{m=0}^{1}\left\|\varphi_{m}\right\|_{\stackrel{\circ}{2, \mu}_{2, \mu}^{1 / 2}\left(\Lambda ; \gamma_{1}\right)} ; \\
& \|u\|_{W_{2, \mu}^{1}(\Omega)} \leqslant c \sum_{m=0}^{1}\left\|g_{m}\right\|_{\stackrel{\circ}{W}_{2, \mu}^{-1 / 2}\left(\Lambda ; \gamma_{1}\right)} ; \\
& \|u\|_{W_{2, \mu}^{1}(\Omega)} \leqslant\left(c_{0}\left\|\varphi_{0}\right\|_{\stackrel{\circ}{W}_{2, \mu}^{1 / 2}\left(\Lambda ; \gamma_{1}\right)}+c_{1}\left\|g_{1}\right\|_{\stackrel{\circ}{2, \mu}_{2}^{-1 / 2}\left(\Lambda ; \gamma_{1}\right)}\right),
\end{aligned}
$$

где постоянные $c, c_{0}$ и $c_{1}$ не зависят от $\mu$.

Сформулируем еше, исходя из всех предыдущих определений, определения функционалов $\frac{\partial u}{\partial \boldsymbol{n}}\left(x^{\prime}, a_{m}\right), m=0,1$, от о.р. задачи $(6.1),(6.2),(6.22 \mathrm{a})$, а также функционал $\frac{\partial u}{\partial \boldsymbol{n}}\left(x^{\prime}, a_{0}\right)$ от о.р. задачи $(6.1),(6.2),(6.22 \mathrm{~B})$. Если $u(x)-$ o.p. задачи $(6.1)$, $(6.2),(6.22 \mathrm{a})$, то согласно определениям 3.2 и $\left.3.3 \frac{\partial u}{\partial \nu}\right|_{\Gamma \backslash \bar{\Gamma}_{2}}$ представляет собой функционал из пространства $W_{2, \mu}^{-1 / 2}\left(\Gamma \backslash \bar{\Gamma}_{2} \rightarrow \Gamma\right)$, действуюший на произвольную $\psi(x) \in W_{2, \mu}^{1 / 2}\left(\Gamma \backslash \bar{\Gamma}_{2} \rightarrow \Gamma\right)$ по формуле

$$
\left\langle\left.\frac{\partial u}{\partial \nu}\right|_{\Gamma \backslash \bar{\Gamma}_{2}}, \psi\right\rangle_{\Gamma \backslash \bar{\Gamma}_{2}}=a[u, \overline{l \psi}]_{\mu}
$$

где $l \psi \in W_{2, \mu}^{1}(\Omega)$ - произвольное продолжение функции $\psi:\left.l \psi\right|_{\Gamma \backslash \bar{\Gamma}_{2}}=\psi$ (такие продолжения сушествуют в силу теоремы 2.4 и определения пространства $\left.W_{2, \mu}^{1 / 2}\left(\Gamma \backslash \bar{\Gamma}_{2} \rightarrow \Gamma\right)\right)$. Значения, $\left.\frac{\partial u}{\partial \boldsymbol{n}}\right|_{\Lambda_{m}}, m=0,1$, естественно определить как сужение функционала $\left.\frac{\partial u}{\partial \nu}\right|_{\Gamma \backslash \bar{\Gamma}_{2}}$ на подпространство

$$
W_{2, \mu}^{1 / 2}\left(\Gamma \backslash \bar{\Gamma}_{2} \rightarrow \Gamma\right) \cap \stackrel{\circ}{W_{2, \mu}^{1 / 2}}\left(\Gamma \backslash \bar{\Gamma}_{2} ; \Gamma_{1} \cup \Lambda_{1-m}\right)=\stackrel{\circ}{W_{2, \mu}^{1 / 2}}\left(\Lambda_{m} \rightarrow \Gamma ; \Gamma_{1} \cup \Lambda_{1-m}\right) .
$$

Поэтому, используя еще леммы $6.4,6.5 .1$ и определение 6.2 , окончательно приходим к следующему определению.

ОПРЕДЕЛЕниЕ 6.3. Под значением нормальных производных $\frac{\partial u}{\partial \boldsymbol{n}}\left(x^{\prime}, a_{m}\right)$, $m=0,1$, от о.р. задачи $(6.1),(6.2),(6.22 \mathrm{a}), \frac{\partial u}{\partial \boldsymbol{n}}\left(x^{\prime}, a_{0}\right)$ от о.р. задачи $(6.1),(6.3)$, (6.22в) будем понимать функционалы из $\stackrel{\circ}{W_{2, \mu}^{-1 / 2}}\left(\Lambda ; \gamma_{1}\right)$, действуюшие на произвольную функцию $\psi(x) \in \stackrel{\circ}{W_{2}^{1 / 2}}\left(\Lambda ; \gamma_{1}\right)$ по формулам

$$
\left\langle\frac{\partial u}{\partial \boldsymbol{n}}\left(x^{\prime}, a_{m}\right), \psi\left(x^{\prime}\right)\right\rangle_{\Lambda}=a\left[u, \overline{l_{m} \psi}\right]_{\mu}
$$


где $l_{m} \psi(x)$ - произвольная функция из $\stackrel{\circ}{W} \underset{2, \mu}{1}\left(\Omega ; \Gamma_{1} \cup \Lambda_{1-m}\right)$, удовлетворяющая условию $l_{m} \psi\left(x^{\prime}, a_{m}\right)=\psi\left(x^{\prime}\right)$.

Такое определение нормальной производной $\frac{\partial u}{\partial \boldsymbol{n}}\left(x^{\prime}, a_{0}\right)$ от о.р. задачи $(6.1),(6.2)$, (6.22в) получается рассуждениями, совершенно аналогичными рассуждениями, приведенным перед этим определением для о.р. задачи (6.1), (6.2), (6.22a).

Заметим, что независимость данного определения от выбора продолжения $l_{m} \psi$ следует из рассуждений, приводящих к определению 6.3; она может быть проверена и непосредственно, исходя из определений (6.24a) и (6.24в) о.р. этих задач.

Для так определенных $\frac{\partial u}{\partial \boldsymbol{n}}\left(x^{\prime}, a_{m}\right)$ справедливы оценки

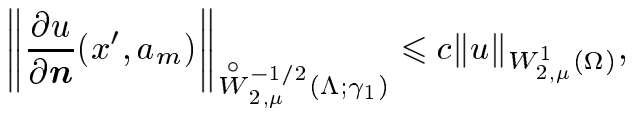

где постоянную $c$ можно взять не зависящей от $\mu$. В самом деле, в силу леммы 6.4 функция $\psi_{m}(x)$ на $\Lambda_{m}$ такая, что $\psi_{m}\left(x^{\prime}, a_{m}\right)=\psi\left(x^{\prime}\right)$, принадлежит $\stackrel{\circ}{W}_{2, \mu}^{1 / 2}\left(\Lambda_{m} \rightarrow \Gamma ; \Gamma_{1} \cup \Lambda_{1-m}\right)$, и при этом

$$
\left\|\psi_{m}\right\|_{\stackrel{\circ}{W}_{2, \mu}^{1 / 2}\left(\Lambda_{m} \rightarrow \Gamma ; \Gamma_{1} \cup \Lambda_{1-m}\right)} \leqslant c\left\|\psi\left(x^{\prime}\right)\right\|_{\stackrel{\circ}{W}_{2, \mu}^{1 / 2}\left(\Lambda ; \gamma_{1}\right)}
$$

с постоянной $c$, не зависящей от $\mu$. В силу определения $(5.6)$ для $\psi_{m}(x)$ найдется такая функция $\lambda \psi_{m}(x) \in W_{2, \mu}^{1 / 2}(\Gamma)$, что $\left.\lambda \psi_{m}\right|_{\Lambda_{m}}=\psi_{m},\left.\lambda \psi_{m}\right|_{\Gamma_{1} \cup \Lambda_{1-m}}=0$ и

$$
\left\|\lambda \psi_{m}\right\|_{W_{2, \mu}^{1 / 2}(\Gamma)} \leqslant 2 c\left\|\psi\left(x^{\prime}\right)\right\|_{W_{2, \mu}^{1 / 2}\left(\Lambda ; \gamma_{1}\right)}
$$

В силу же теоремы 2.4 функция $l_{m} \psi=l_{\Gamma}^{\Omega} \lambda \psi_{m} \in \stackrel{\circ}{{ }^{\circ}} \frac{1}{2, \mu}\left(\Omega ; \Gamma_{1} \cup \Lambda_{1-m}\right)$, $l_{m} \psi\left(x^{\prime}, a_{m}\right)=\psi\left(x^{\prime}\right)$ и

$$
\left\|l_{m} \psi\right\|_{W_{2, \mu}^{1}(\Omega)} \leqslant \widetilde{c}\left\|\psi\left(x^{\prime}\right)\right\|_{W_{2, \mu}^{1 / 2}\left(\Lambda ; \gamma_{1}\right)} .
$$

Использование теперь в (6.26) такого продолжения $l_{m} \psi$ вместе с оценкой (3.12) приводит к оценке (6.27).

Перейдем к обоснованию метода Фурье для рассматриваемых задач. Обозначим через

$$
\left\{e_{k}\left(x^{\prime}\right)\right\}_{k=1}^{\infty}
$$

ортонормированный базис в $L_{2}(\Lambda)$ из о.с.ф. оператора $L^{\prime}\left(x^{\prime}, D^{\prime}\right)$, определяемого (6.4б), при однородных смешанных граничных условиях

$$
\left.u\left(x^{\prime}\right)\right|_{\gamma_{1}}=0,\left.\quad\left(\frac{\partial u}{\partial \nu}\left(x^{\prime}\right)+\sigma\left(x^{\prime}\right) u\left(x^{\prime}\right)\right)\right|_{\gamma_{2}}=0
$$


каждая о.с.ф. $e_{k}\left(x^{\prime}\right)$ отвечает с.з. $\lambda_{k}$, и будем предполагать нумерацию элементов базиса (6.28) такой, что $\lambda_{k} \leqslant \lambda_{k+1} \forall k \in \mathbb{N}$ (теорема 4.1 устанавливает существование такого базиса).

При решении каждой из рассматриваемых смешанных задач граничные данные - функции $\varphi_{m}\left(x^{\prime}\right)$ или/и функционалы $g_{m}\left(x^{\prime}\right)$ разлагаются в ряды Фурье вида:

$$
\begin{aligned}
& \text { для } \varphi\left(x^{\prime}\right) \in \stackrel{\circ}{W_{2, \mu}^{1 / 2}}\left(\Lambda ; \gamma_{1}\right) \\
& \qquad\left(x^{\prime}\right)=\sum_{k=1}^{\infty} c_{k}(\varphi) e_{k}\left(x^{\prime}\right), \text { где } c_{k}(\varphi)=\left(\varphi, e_{k}\right)_{L_{2}(\Lambda)}, \\
& \text { для } g\left(x^{\prime}\right) \in \stackrel{\circ}{W_{2, \mu}^{-1 / 2}}\left(\Lambda ; \gamma_{1}\right) \\
& g\left(x^{\prime}\right)=\sum_{k=1}^{\infty} c_{k}(g) e_{k}\left(x^{\prime}\right), \text { где } c_{k}(g)=\left\langle g\left(x^{\prime}\right), \overline{e_{k}\left(x^{\prime}\right)}\right\rangle_{\Lambda},
\end{aligned}
$$

по системе о.с.ф. (6.28). Решение каждой из задач ищется в виде ряда

$$
u(x)=\sum_{k=1}^{\infty} Y_{k}\left(x_{n}\right) e_{k}\left(x^{\prime}\right) .
$$

Формальная подстановка таких рядов в уравнение и граничные условия приводит к следуюшим формальных разложениям для решения $u(x)$ каждой из этих задач, соответственно,

$$
\begin{aligned}
& u(x) \sim \sum_{k=1}^{\infty} \sum_{m=0}^{1} X_{k}^{m}\left(x_{n}\right) c_{k}\left(\varphi_{m}\right) e_{k}\left(x^{\prime}\right) \\
& u(x) \sim \sum_{k=1}^{\infty} \sum_{m=0}^{1} X_{k}^{m}\left(x_{n}\right) c_{k}\left(g_{m}\right) e_{k}\left(x^{\prime}\right) ; \\
& u(x) \sim \sum_{k=1}^{\infty}\left(X_{k}^{0}\left(x_{n}\right) c_{k}\left(\varphi_{0}\right)+X_{k}^{1}\left(x_{n}\right) c_{k}\left(g_{1}\right)\right) e_{k}\left(x^{\prime}\right),
\end{aligned}
$$

где $X_{k}^{m}\left(x_{n}\right)$ - решения уравнений

$$
-D_{n}^{2} X_{k}^{m}\left(x_{n}\right)+\left(\lambda_{k}+\mu^{2}\right) X_{k}^{m}\left(x_{n}\right)=0
$$

удовлетворяющие, соответственно, следующим краевым условиям

$$
\begin{aligned}
& X_{k}^{0}\left(a_{0}\right)=1, \quad X_{k}^{0}\left(a_{1}\right)=0, \quad X_{k}^{1}\left(a_{0}\right)=0, \quad X_{k}^{1}\left(a_{1}\right)=1 ;(6.34 \mathrm{a}) \\
& -D_{n} X_{k}^{0}\left(a_{0}\right)=1, \quad D_{n} X_{k}^{0}\left(a_{1}\right)=0, \quad D_{n} X_{k}^{1}\left(a_{0}\right)=0, \quad D_{n} X_{k}^{1}\left(a_{1}\right)=1 ;(6.34 \sigma) \\
& X_{k}^{0}\left(a_{0}\right)=1, \quad D_{n} X_{k}^{0}\left(a_{1}\right)=0, \quad X_{k}^{1}\left(a_{0}\right)=0, \quad D_{n} X_{k}^{1}\left(a_{1}\right)=1 . \quad\left(6.34_{\mathrm{B}}\right)
\end{aligned}
$$


ТЕОРема 6.1. Пусть выполнены все условия, приведенные в начале 6 , $u \mu \geqslant \mu_{0}$, әде $\mu_{0}$ - достаточно большое. Тогда справедливь следующие утверждения.

1. $\forall \varphi\left(x^{\prime}\right) \in \stackrel{\circ}{{ }^{1 / 2}} \underset{2, \mu}{ }\left(\Lambda ; \gamma_{1}\right)$ ряд Фурве (6.30) сходится $\kappa \varphi\left(x^{\prime}\right)$ в пространстве $\stackrel{\circ}{W} \underset{2, \mu}{1 / 2}\left(\Lambda ; \gamma_{1}\right)$. Эквивалентным скалярным произведением в әтом пространстве двух функиий $\varphi_{1}\left(x^{\prime}\right)$ и $\varphi_{2}\left(x^{\prime}\right)$ является скалярное произведение:

$$
\left(\varphi_{1}, \varphi_{2}\right) \underset{\stackrel{\circ}{W}_{2, \mu}^{1 / 2}\left(\Lambda ; \gamma_{1}\right)}{\sim}=\sum_{k=1}^{\infty}\left(\lambda_{k}+\mu^{2}\right)^{1 / 2} c_{k}\left(\varphi_{1}\right) \overline{c_{k}\left(\varphi_{2}\right)},
$$

относительно которого система о.с.ф. (6.28) ортогональна.

$\forall g\left(x^{\prime}\right) \in \stackrel{\circ}{W} \underset{2, \mu}{-1 / 2}\left(\Lambda ; \gamma_{1}\right)$ ряд Фурье (6.31) сходится $к g\left(x^{\prime}\right)$ в пространстве $\stackrel{\circ}{W}_{2, \mu}^{-1 / 2}\left(\Lambda ; \gamma_{1}\right)$. Эквивалентным скалярным произведением в әтом пространстве двух функционалов $g_{1}\left(x^{\prime}\right)$ и $g_{2}\left(x^{\prime}\right)$ является скалярное произведение

$$
\left(g_{1}, g_{2}\right) \underset{\stackrel{\circ}{W_{2, \mu}^{-1 / 2}\left(\Lambda ; \gamma_{1}\right)}}{\sim}=\sum_{k=1}^{\infty}\left(\lambda_{k}+\mu^{2}\right)^{-1 / 2} c_{k}\left(g_{1}\right) \overline{c_{k}\left(g_{2}\right)},
$$

относительно которого система (6.28) также ортогональна.

2. При выполнении (6.23) каждый из формальных рядов (6.32a)-(6.32в) сходится в $W_{2, \mu}^{1}(\Omega)$ к обобщенному решению соответствующей смешанной задачи (6.1), (6.2), (6.22a)-(6.22в).

3. Значения $\frac{\partial u}{\partial \boldsymbol{n}}\left(x^{\prime}, a_{j}\right), j=0,1$, от о.р. задачи (6.1), (6.2), (6.22a), а такәке $\frac{\partial u}{\partial \boldsymbol{n}}\left(x^{\prime}, a_{0}\right)$ от о.р. задачи (6.1), (6.2), (6.22в) представимьи, соответственно, рядами

$$
\begin{gathered}
\sum_{k=1}^{\infty} \sum_{m=0}^{1}(-1)^{j+1} D_{n} X_{k}^{m}\left(a_{j}\right) c_{k}\left(\varphi_{m}\right) e_{k}\left(x^{\prime}\right), \quad j=0,1, \\
-\sum_{k=1}^{\infty}\left(D_{n} X_{k}^{0}\left(a_{0}\right) c_{k}\left(\varphi_{0}\right)+D_{n} X_{k}^{1}\left(a_{0}\right) c_{k}\left(g_{1}\right)\right) e_{k}\left(x^{\prime}\right),
\end{gathered}
$$

сходящимися в $\stackrel{\circ}{W}_{2, \mu}^{-1 / 2}\left(\Lambda ; \gamma_{1}\right)$ и представляющими собой рядь Фурье для этих функиионалов. Значения $u\left(x^{\prime}, a_{j}\right), j=0,1$, от о.р. задачи (6.1), (6.2), (6.22б) и и $\left(x^{\prime}, a_{1}\right)$ от о.р. задачи (6.1), (6.2), (6.22в) представимы, соответственно, рядами

$$
\begin{aligned}
& \sum_{k=1}^{\infty} \sum_{m=0}^{1} X_{k}^{m}\left(a_{j}\right) c_{k}\left(g_{m}\right) e_{k}\left(x^{\prime}\right), \quad j=0,1, \\
& \sum_{k=1}^{\infty}\left(X_{k}^{0}\left(a_{1}\right) c_{k}\left(\varphi_{0}\right)+X_{k}^{1}\left(a_{1}\right) c_{k}\left(g_{1}\right)\right) e_{k}\left(x^{\prime}\right),
\end{aligned}
$$

сходящимися в $\stackrel{\circ}{\mathrm{W}} \underset{2, \mu}{1 / 2}\left(\Lambda ; \gamma_{1}\right)$ и представляющими собой рядь Фурье для этих граничных значений. 
ДокАЗАТЕЛЬСтво. Сначала проверим, что всякая функция

$$
E_{k}\left(x^{\prime}, x_{n}\right)=X_{k}\left(x_{n}\right) e_{k}\left(x^{\prime}\right),
$$

где $X_{k}\left(x_{n}\right)$ - некоторое решение уравнения $(6.33)$ на $\left(a_{0}, a_{1}\right)$, является о.р. любой из задач: (6.1) , (6.2) плюс один из следующих наборов граничных условий на торцах $\Lambda_{0}$ и $\Lambda_{1}$ :

$$
\begin{gathered}
E_{k}\left(x^{\prime}, a_{j}\right)=X_{k}\left(a_{j}\right) e_{k}\left(x^{\prime}\right), \quad j=0,1, \\
\frac{\partial E_{k}}{\partial \boldsymbol{n}}\left(x^{\prime}, a_{j}\right)=(-1)^{j+1} D_{n} X_{k}\left(a_{j}\right) e_{k}\left(x^{\prime}\right), \quad j=0,1, \\
E_{k}\left(x^{\prime}, a_{0}\right)=X_{k}\left(a_{0}\right) e_{k}\left(x^{\prime}\right), \quad \frac{\partial E_{k}}{\partial \boldsymbol{n}}\left(x^{\prime}, a_{1}\right)=D_{n} X_{k}\left(a_{1}\right) e_{k}\left(x^{\prime}\right),
\end{gathered}
$$

например, задачи (6.1), (6.2), (6.40в) (эта задача содержит оба вида граничных условий на торцах). Для остальных задач проверка аналогична.

Для этого воспользуемся следующим элементарным фактом. Пусть $\Omega=\Omega^{\prime} \times \Omega^{\prime \prime}$ - область в $\mathbb{R}^{n_{1}+n_{2}}$, где $\Omega^{\prime}$ и $\Omega^{\prime \prime}$ - области в $\mathbb{R}^{n_{1}}$ и $\mathbb{R}^{n_{2}}$, соответственно. Тогда, если $u\left(x^{\prime}\right) \in W_{2, \mu}^{1}\left(\Omega^{\prime}\right), v\left(x^{\prime \prime}\right) \in W_{2, \mu}^{1}\left(\Omega^{\prime \prime}\right)$, то $u\left(x^{\prime}\right) v\left(x^{\prime \prime}\right) \in W_{2, \mu}^{1}(\Omega)$, и справедлива оценка

$$
\|u v\|_{W_{2, \mu}^{1}(\Omega)} \leqslant \mu^{-1}\|u\|_{W_{2, \mu}^{1}\left(\Omega^{\prime}\right)}\|v\|_{W_{2, \mu}^{1}\left(\Omega^{\prime \prime}\right)} .
$$

Поэтому $E_{k}\left(x^{\prime}, x_{n}\right) \in W_{2, \mu}^{1}(\Omega)$. Далее, покажем, что в смысле следов

$$
\left.E_{k}\left(x^{\prime}, x_{n}\right)\right|_{\Gamma_{1}}=0, \quad E_{k}\left(x^{\prime}, a_{j}\right)=X_{k}\left(a_{j}\right) e_{k}\left(x^{\prime}\right), \quad j=0,1 .
$$

В силу леммы 2.3 для о.с.ф. $e_{k}\left(x^{\prime}\right)$ найдется последовательность функций $e_{k, \nu}\left(x^{\prime}\right) \in C^{\infty}(\bar{\Lambda})$ таких, что $\left.e_{k, \nu}\left(x^{\prime}\right)\right|_{\gamma_{1}}=0$ и $e_{k, \nu}\left(x^{\prime}\right) \rightarrow e_{k}\left(x^{\prime}\right)$ в $W_{2, \mu}^{1}(\Lambda)$ при $\nu \rightarrow \infty$. Тогда $E_{k, \nu}\left(x^{\prime}, x_{n}\right)=X_{k}\left(x_{n}\right) e_{k, \nu}\left(x^{\prime}\right) \in C^{\infty}(\bar{\Omega})$, и в силу $(6.41)$ $E_{k, \nu}\left(x^{\prime}, x_{n}\right) \rightarrow E_{k}\left(x^{\prime}, x_{n}\right)$ в $W_{2, \mu}^{1}(\Omega)$ при $\nu \rightarrow \infty$.

Поскольку $\left.E_{k, \nu}\left(x^{\prime}, x_{n}\right)\right|_{\Gamma_{1}}=0$, согласно определению следа получаем первое равенство в (6.42). Кроме того, $E_{k, \nu}\left(x^{\prime}, a_{j}\right)=X_{k}\left(a_{j}\right) e_{k, \nu}\left(x^{\prime}\right) \rightarrow X_{k}\left(a_{j}\right) e_{k}\left(x^{\prime}\right)$ в $W_{2, \mu}^{1}(\Lambda)$, а потому и в $L_{2}(\Lambda)$ при $\nu \rightarrow \infty$. С другой стороны, по определению следа $E_{k, \nu}\left(x^{\prime}, a_{j}\right) \rightarrow E_{k}\left(x^{\prime}, a_{j}\right)$ в $W_{2, \mu}^{1 / 2}(\Lambda)$, а потому также и в $L_{2}(\Lambda)$ при $\nu \rightarrow \infty$. Отсюда получаем два последних равенства в (6.42). (Заметим, что (6.42) могут быть установлены и без привлечения леммы 2.3 и условия, что $\gamma_{1}$ - липшицев кусок.)

Проверим теперь выполнение $\forall v(x) \in \stackrel{\circ}{W_{2, \mu}^{1}}\left(\Omega ; \Gamma_{1} \cup \Lambda_{0}\right)$ соотношения вида $(6.24 \mathrm{~B})$, а именно:

$$
a\left[E_{k}, v\right]_{\mu}=\int_{\Lambda} D_{n} X_{k}\left(a_{1}\right) e_{k}\left(x^{\prime}\right) \overline{v\left(x^{\prime}, a_{1}\right)} d x^{\prime}
$$

В силу леммы 2.3 это соотношение достаточно проверить $\forall v(x) \in C^{\infty}(\bar{\Omega})$, удовлетворяюшей условию $\left.v(x)\right|_{\overline{\Gamma_{1} \cup \Lambda_{0}}}=0$. Обрашаясь к формулам $(6.9),(6.10)$ и используя определяюшее соотношение вида $(4.3)$ для $e_{k}\left(x^{\prime}\right)$ :

$$
a^{\prime}\left[e_{k}\left(x^{\prime}\right), v\left(x^{\prime}, x_{n}\right)\right]_{\mu}=\left(\lambda_{k}+\mu^{2}\right) \int_{\Lambda} e_{k}\left(x^{\prime}\right) \overline{v\left(x^{\prime}, x_{n}\right)} d x^{\prime}
$$


$\left(v\left(x^{\prime}, x_{n}\right) \in \stackrel{\circ}{W} \underset{2, \mu}{1}\left(\Lambda ; \gamma_{1}\right)\right.$ при каждом фиксированном $\left.x_{n}\right)$, а также теорему Фубини, получаем, что

$$
\begin{aligned}
a\left[E_{k}, v\right]_{\mu}=\left(\lambda_{k}+\mu^{2}\right)\left(E_{k}, v\right)_{L_{2}(\Omega)} & \\
& +\int_{\Lambda} e_{k}\left(x^{\prime}\right)\left(\int_{a_{0}}^{a_{1}} D_{n} X_{k}\left(x_{n}\right) D_{n} \overline{v\left(x^{\prime}, x_{n}\right)} d x_{n}\right) d x^{\prime}
\end{aligned}
$$

Перебрасывая в последнем интеграле по $x_{n}$ интегрированием по частям оператор $D_{n}$ с $\bar{v}$ на $D_{n} X_{k}$ и учитывая при этом, что $v\left(x^{\prime}, a_{0}\right)=0$, а $X_{k}\left(x_{n}\right)$ удовлетворяет уравнению (6.33), получаем справедливость (6.43). Итак, сформулированные свойства функций (6.39) установлены. Отметим, что условие, что $\gamma_{1}$ является липшицевьм куском $\partial \Lambda$, существенно используется именно на этом этапе.

Нетрудно установить, сравнивая определение 6.3 и определение о.p. (6.24б), что имеют место равенства $(6.40 б)$, в которых $\frac{\partial E_{k}}{\partial \boldsymbol{n}}\left(x^{\prime}, a_{j}\right)$ - уже значения на торцах нормальной производной от о.р. $E_{k}$ какой-либо из задач $(6.1),(6.2),(6.40 \mathrm{a})$ или $(6.40 \mathrm{~B})$.

Перейдем теперь к доказательству первой части теоремы 6.1. Рассмотрим смешанную задачу $(6.1),(6.2),(6.3$ в $)$ с $\varphi_{0}(x)=0$ и обозначим через $S_{\mu}$ оператор Пуанкаре-Стеклова, соответствующий этой задаче (см. определение 5.1). Обозначим

$$
E_{k}^{m}\left(x^{\prime}, x_{n}\right)=X_{k}^{m}\left(x_{n}\right) e_{k}\left(x^{\prime}\right), \quad m=0,1
$$

где $X_{k}^{m}\left(x_{n}\right)$ - решения уравнения (6.33) с граничньпи условиями (6.34в). В силу предыдушего $E_{k}^{1}\left(x^{\prime}, x_{n}\right)$ - решение задачи $(6.1),(6.2)$ плюс граничные условия

$$
E_{k}^{1}\left(x^{\prime}, a_{0}\right)=0, \quad \frac{\partial E_{k}^{1}}{\partial \boldsymbol{n}}\left(x^{\prime}, a_{1}\right)=e_{k}\left(x^{\prime}\right) .
$$

Обозначим, далее, через $\varepsilon_{k}(x)$ функции на $\Lambda_{1}$ такие, что $\varepsilon_{k}\left(x^{\prime}, a_{1}\right)=e_{k}\left(x^{\prime}\right)$. Тогда $E_{k}^{1}\left(x^{\prime}, x_{n}\right)$ представляет собой решение задачи $(6.1),(6.2),(6.3 \mathrm{~B})$ с $\varphi_{0}(x)=0$ и с $g_{1}(x)=\varepsilon_{k}(x)$ (точнее с функционалом $g_{1}(x)$, порождаемым функцией $\varepsilon_{k}(x)$ : $\left.\left\langle g_{1}, \psi\right\rangle_{\Lambda_{1}}=\int_{\Lambda_{1}} \varepsilon_{k}(x) \psi(x) d s\right)$. Но согласно (6.40а) $E_{k}^{1}\left(x^{\prime}, a_{1}\right)=X_{k}^{1}\left(a_{1}\right) e_{k}\left(x^{\prime}\right)$.

Отсюда

$$
S_{\mu} \varepsilon_{k}(x)=X_{k}^{1}\left(a_{1}\right) \varepsilon_{k}(x), \quad k \in \mathbb{N} .
$$

Таким образом, система функций $\left\{\varepsilon_{k}(x)\right\}_{k=1}^{\infty}$ представляет собой ортонормированный базис в $L_{2}\left(\Lambda_{1}\right)$ из собственных функций оператора Пуанкаре-Стеклова $S_{\mu}$ на $\Lambda_{1}$, отвечающих характеристическим значениям

$$
\nu_{k}=\left(X_{k}^{1}\left(a_{1}\right)\right)^{-1}=\sqrt{\lambda_{k}+\mu^{2}} \operatorname{cth} \sqrt{\lambda_{k}+\mu^{2}}\left(a_{1}-a_{0}\right) .
$$

Поэтому утверждения первой части теоремы 6.1 являются непосредственными следствиями теоремы 5.2 и лемм 6.4 и 6.5.1.

В самом деле, пусть $\varphi\left(x^{\prime}\right)$ - произвольная функция из $\stackrel{\circ}{W} \underset{2, \mu}{1 / 2}\left(\Lambda ; \gamma_{1}\right)$. Обозначим через $\widehat{\varphi}(x)$ функцию на $\Lambda_{1}$ такую, что $\widehat{\varphi}\left(x^{\prime}, a_{1}\right)=\varphi\left(x^{\prime}\right)$. Тогда в силу леммы 6.4 
$\widehat{\varphi}(x) \in \stackrel{\circ}{W}{ }_{2, \mu}^{1 / 2}\left(\Lambda_{1} \rightarrow \Gamma ; \Gamma_{1} \cup \Lambda_{0}\right)$, нормы функции $\widehat{\varphi}(x)$ в последнем пространстве и функции $\varphi\left(x^{\prime}\right)$ в пространстве $\stackrel{\circ}{{ }_{2}^{1 / 2}} \underset{2, \mu}{ }\left(\Lambda ; \gamma_{1}\right)$ эквивалентны. По теореме $5.2 \widehat{\varphi}(x)$ представима сходящимся в пространстве $\stackrel{\circ}{W} \underset{2, \mu}{1 / 2}\left(\Lambda_{1} \rightarrow \Gamma ; \Gamma_{1} \cup \Lambda_{0}\right)$ рядом Фурье

$$
\sum_{k=1}^{\infty} c_{k}(\widehat{\varphi}) \varepsilon_{k}(x)
$$

где

$$
c_{k}(\widehat{\varphi})=\int_{\Lambda_{1}} \widehat{\varphi}(x) \overline{\varepsilon_{k}(x)} d s=\int_{\Lambda} \varphi\left(x^{\prime}\right) \overline{e_{k}\left(x^{\prime}\right)} d x^{\prime}=c_{k}(\varphi)
$$

являются в то же время и коэффициентами Фурье функции $\varphi\left(x^{\prime}\right)$ по системе о.с.ф̆. (6.28). Тогда, ряд

$$
\sum_{k=1}^{\infty} c_{k}(\widehat{\varphi}) \varepsilon_{k}\left(x^{\prime}, a_{1}\right)=\sum_{k=1}^{\infty} c_{k}(\varphi) e_{k}\left(x^{\prime}\right)
$$

(представляюший собой ряд Фурье (6.30)) опять же в силу леммы 6.4 сходится по норме пространства $\stackrel{\circ}{\underset{W}{2}} \underset{2, \mu}{1 / 2}\left(\Lambda ; \gamma_{1}\right)$ к функции $\widehat{\varphi}\left(x^{\prime}, a_{1}\right)=\varphi\left(x^{\prime}\right)$. Наконец, использование формулы (5.20а) и леммы 6.4 дает, что эквивалентным скалярным произведением в $\stackrel{\circ}{W} \underset{2, \mu}{1 / 2}\left(\Lambda ; \gamma_{1}\right)$ двух функций $\varphi_{1}\left(x^{\prime}\right)$ и $\varphi_{2}\left(x^{\prime}\right)$ является форма

$$
\left(\varphi_{1}, \varphi_{2}\right)_{\substack{W_{2, \mu}^{1 / 2}\left(\Lambda ; \gamma_{1}\right) \\ *}}^{*}=\sum_{k=1}^{\infty} \nu_{k} c_{k}\left(\widehat{\varphi}_{1}\right) \overline{c_{k}\left(\widehat{\varphi}_{2}\right)}=\sum_{k=1}^{\infty} \nu_{k} c_{k}\left(\varphi_{1}\right) \overline{c_{k}\left(\varphi_{2}\right)}
$$

где $\widehat{\varphi}_{i}(x), i=1,2$, определяются по $\varphi_{i}\left(x^{\prime}\right)$ так же, как $\widehat{\varphi}(x)$ определялась вьше по $\varphi\left(x^{\prime}\right)$. В силу эквивалентности, $\nu_{k} \sim \sqrt{\lambda_{k}+\mu^{2}}$, последнее скалярное произведение эквивалентно скалярному произведению (6.35).

Далее, пусть $g\left(x^{\prime}\right)$ - произвольньй функционал из $\stackrel{\circ}{\underset{W}{2}} \underset{2, \mu}{-1 / 2}\left(\Lambda ; \gamma_{1}\right)$. Построим по $g\left(x^{\prime}\right)$ функционал $\widehat{g}(x) \in \stackrel{\circ}{\underset{W}{2}}-1 / 2\left(\Lambda_{1} \rightarrow \Gamma ; \Gamma_{1} \cup \Lambda_{0}\right)$ такой, что в смысле определения $5.2 \widehat{g}\left(x^{\prime}, a_{1}\right)=g\left(x^{\prime}\right)$. В силу (6.21) такой функционал $\widehat{g}(x)$ определяется формулой

$$
\langle\widehat{g}(x), \widehat{\varphi}(x)\rangle_{\Lambda_{1}}=\left\langle g\left(x^{\prime}\right), \varphi\left(x^{\prime}\right)\right\rangle_{\Lambda},
$$

где $\widehat{\varphi}\left(x^{\prime}, a_{1}\right)=\varphi\left(x^{\prime}\right)$. В силу теоремы 5.2 функционал $\widehat{g}(x)$ представи́м сходящимся в пространстве $\stackrel{\circ}{\underset{W}{W}} \underset{2, \mu}{-1 / 2}\left(\Lambda_{1} \rightarrow \Gamma ; \Gamma_{1} \cup \Lambda_{0}\right)$ рядом Фурье

$$
\sum_{k=1}^{\infty} c_{k}(\widehat{g}) \varepsilon_{k}(x)
$$

где

$$
c_{k}(\widehat{g})=\left\langle\widehat{g}(x), \overline{\varepsilon_{k}(x)}\right\rangle_{\Lambda_{1}}=\left\langle g\left(x^{\prime}\right), \overline{e_{k}\left(x^{\prime}\right)}\right\rangle_{\Lambda}
$$


являются, как мы видим, и коэффициентами Фурье функционала $g\left(x^{\prime}\right)$ по системе (6.26). Но тогда в силу леммы 6.5.1 ряд

$$
\sum_{k=1}^{\infty} c_{k}(\widehat{g}) \varepsilon_{k}\left(x^{\prime}, a_{1}\right)=\sum_{k=1}^{\infty} c_{k}(g) e_{k}\left(x^{\prime}\right)
$$

сходится в $\left.\stackrel{\circ}{W}-1 / 2, \Lambda ; \gamma_{1}\right)$ к функционалу $g\left(x^{\prime}\right)$. Точно так же, на основании формулы (5.20б) с помощью леммы 6.5.1 устанавливается, что эквивалентным скалярным произведением в $\stackrel{\circ}{W} \underset{2, \mu}{-1 / 2}\left(\Lambda ; \gamma_{1}\right)$ является скалярное произведение (6.34).

Перейдем к доказательству второй части теоремы 6.1. Проведем его, например, для задачи $(6.1),(6.2),(6.22 \mathrm{~B})$. Пусть $u\left(x^{\prime}\right)$ - о.р. этой задачи. В силу установленного выше относительно функций (6.39), функции (6.44) являются о.р. задачи $(6.1),(6.2)$ плюс граничные условия

$$
E_{k}^{m}\left(x^{\prime}, a_{0}\right)=\delta_{0}^{m} e_{k}\left(x^{\prime}\right), \quad \frac{\partial E_{k}^{m}}{\partial \boldsymbol{n}}\left(x^{\prime}, a_{1}\right)=\delta_{1}^{m} e_{k}\left(x^{\prime}\right)
$$

где $\delta_{j}^{m}$ - символ Кронекера. Поэтому $N$-ая частичная сумма $u_{N}(x)$ ряда $(6.32$ в) представляет собой решение задачи (6.1), (6.2), (6.22в) с $\varphi_{0}\left(x^{\prime}\right)$ и $g_{1}\left(x^{\prime}\right)$, замененными, соответственно, на

$$
\varphi_{0, N}\left(x^{\prime}\right)=\sum_{k=1}^{N} c_{k}\left(\varphi_{0}\right) e_{k}\left(x^{\prime}\right) \text { и } g_{1, N}\left(x^{\prime}\right)=\sum_{k=1}^{N} c_{k}\left(g_{1}\right) e_{k}\left(x^{\prime}\right) .
$$

Тогда $\left(u(x)-u_{N}(x)\right)$ представляет собой решение задачи $(6.1),(6.2),(6.22 \mathrm{~B})$ с $\varphi_{0}\left(x^{\prime}\right)$, замененной на $\left(\varphi_{0}\left(x^{\prime}\right)-\varphi_{0, N}\left(x^{\prime}\right)\right)$, и с $g_{1}\left(x^{\prime}\right)$, замененным на $\left(g_{1}\left(x^{\prime}\right)-\right.$ $\left.g_{1, N}\left(x^{\prime}\right)\right)$. Но вследствие уже установленной части 1 теоремы $6.1 \varphi_{0, N}\left(x^{\prime}\right) \rightarrow$

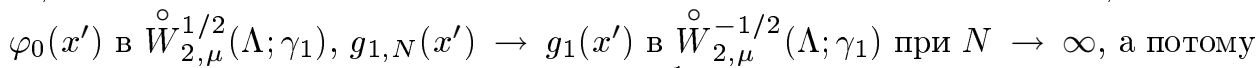
оценка $(6.25 \mathrm{~B})$ дает, что $u_{N}(x) \rightarrow u(x)$ в $W_{2, \mu}^{1}(\Omega)$ при $N \rightarrow \infty$. Аналогично получаем обоснование метода Фурье и для других задач.

Доказательство третьей части теоремы 6.1 проводится непосредственно на основании определений с использованием соотношений (6.40а) и (6.40б), в которых $\frac{\partial E_{k}}{\partial \boldsymbol{n}}\left(x^{\prime}, a_{j}\right)$ - значения нормальных производных от о.р. $E_{k}$, теоремы 2.3 вложения и оценок $(6.27)$, в которых $u(x)$ заменяется на $\left(u(x)-u_{N}(x)\right)$, где $u_{N}(x)-N$-ые частичные суммы рядов (6.32).

В заключение сделаем два замечания.

1. В случае, ког да $\gamma_{1}=\varnothing$, пространство $\stackrel{0}{W_{2, \mu}^{1 / 2}}\left(\Lambda ; \gamma_{1}\right)$ преврашается в пространство $W_{2, \mu}^{1 / 2}(\Lambda)$. Для случая, когда $\gamma_{1}=\partial \Lambda$, можно показать, что пространство $\stackrel{\circ}{W} \underset{2, \mu}{1 / 2}(\Lambda ; \partial \Lambda)$ (как множество функций) совпадает с пространством $\stackrel{\circ}{W_{2, \mu}^{1 / 2}}(\Lambda)$, определяемым как подмножество функций $u\left(x^{\prime}\right)$ из $W_{2, \mu}^{1 / 2}(\Lambda)$ таких, что функции

$$
\stackrel{\circ}{u}\left(x^{\prime}\right)= \begin{cases}u\left(x^{\prime}\right) & \text { при } x^{\prime} \in \Lambda, \\ 0 & \text { при } x^{\prime} \in \mathbb{R}^{n-1} \backslash \Lambda\end{cases}
$$


принадлежат пространству $W_{2, \mu}^{1 / 2}\left(\mathbb{R}^{n}\right)$, с нормой

$$
\|u\|_{\stackrel{\circ}{W}_{2, \mu}^{1 / 2}(\Lambda)}=\|\stackrel{\circ}{u}\|_{W_{2, \mu}^{1 / 2}\left(\mathbb{R}^{n-1}\right)}
$$

При этом нормировки $\stackrel{\circ}{W} \underset{2, \mu}{1 / 2}(\Lambda ; \partial \Lambda)$ и $\stackrel{\circ}{W} \underset{2, \mu}{1 / 2}(\Lambda)$ эквивалентны.

2. Часть 1 теоремы 6.1 дает следующее дополнение к теореме 4.1. Для всякой функции $u \in \stackrel{\circ}{\underset{W}{1}} \underset{2, \mu}{1 / 2}\left(\Omega ; \Gamma_{1}\right)$ ряд Фурье $(4.7 \mathrm{a})$ сходится к $u$ в пространстве $\stackrel{\circ}{W} \underset{2, \mu}{1 / 2}\left(\Omega ; \Gamma_{1}\right)$, для всякого функционала $f \in \stackrel{\circ}{W} \underset{2, \mu}{-1 / 2}\left(\Omega ; \Gamma_{1}\right)$ ряд Фурье $(4.7 б)$ сходится к $f$ в пространстве $\stackrel{\circ}{W} \underset{2, \mu}{-1 / 2}\left(\Omega ; \Gamma_{1}\right)$. При достаточно больших $\mu$ эквивалентньпи скалярными произведениями в $\stackrel{\circ}{W} \underset{2, \mu}{1 / 2}\left(\Omega ; \Gamma_{1}\right)$ и в $\stackrel{\circ}{W}-1 / 2\left(\Omega ; \Gamma_{1}\right)$ являются, соответственно, скалярные произведения вида (6.35) и (6.36), в которых теперь $c_{k}\left(\varphi_{j}\right)$ и $c_{k}\left(g_{j}\right)$-коэффициенты Фурье функций $\varphi_{j} \in \stackrel{\circ}{\stackrel{D}{W}_{2, \mu}^{1 / 2}}\left(\Omega ; \Gamma_{1}\right)$ и функционалов $g_{j} \in \stackrel{\circ}{W_{2, \mu}^{-1 / 2}}\left(\Omega ; \Gamma_{1}\right)$ по системе о.с.ф. оператора, фигурируюшего в теореме $4.1, \lambda_{k}-$ с.з. этого оператора.

\section{Список литературы}

1. Агмон II., Дуглис А., Ниренберг Л. Оценки решений эллиптических уравнений вблизи границы. М.: ИЛ, 1962.

2. Schechter M. General boundary value problems for elliptic equations // Comm. Pure and Appl. Math. 1959. V. 12. P. 457-486.

3. Peetre J. Another approach to elliptic boundary problems // Comm. Pure and Appl. Math. 1961. V. 14. P. 711-731.

4. Агранович М. С., Вишик М.И. Эллиптические задачи с параметром и параболические задачи общего вида // УМН. 1964. Т. 19. №3. С. 54-112.

5. Лионс Ж.-Л., Мадженес Э. Неоднородные граничные задачи и их приложения. М.: Мир, 1971.

6. Ройтберг Я. А. Эллиптические задачи с неоднородньми граничньми условиями и локальне повышение гладкости вплоть до границы обобшенных решений // ДАН СССР. 1964. T. 157. № 4. C. 798-801.

7. Nečas J. Les méthodes directes en théorie des équations elliptiques. Prague: Academia, 1967.

8. Кондратьев B.A., Олейник O.A. Краевые задачи для уравнений с частными производнњгми в негладких областях // УМН. 1983. Т. 38. № 2. С. 3-76.

9. Crisvard P. Elliptic problems in nonsmooth domains. London: Pittman, 1985.

10. Zaremba $S$. Sur un problème mixte relatif à l'equation de Laplace // Bull. Acad. Sci. Cracovie. Classe des sciences mathématiques et naturelles. Ser A. 1910. P. 313-344.

11. Schechter $M$. Mixed boundary problem for general elliptic equations // Comm. Pure and Appl. Math. 1960. V. 13. P. 331-354.

12. Peetre J. Mixed problems for higher order elliptic equations in two variables // I. Ann. Scuol. Norm. Sup. Pisa. 1961. V. 15. P. 337-353; // II. Ann. Scuol Norm. Sup. Pisa. 1963. V. 17. P. 1-12.

13. Вишик М.И., Эскин Г.И. Пространства Соболева-Слободецкого переменного порядка с весовьми нормами и их приложения к смешанньм краевьм задачам // Сиб. матем. журн. 1968. Т. 9. № 5. С. 973-997.

14. Эскин Г.И. Краевые задачи для эллиптических псевдодифференциальных уравнений. М.: Наука, 1973. 
15. Лебедев В.И., Агошков В.И. Операторы Пуанкаре-Стеклова и их приложения в анализе. М.: Отдел вычислительной математики АН СССР, 1983.

16. Агошков В.И., Лебедев В.И. Операторы Пуанкаре-Стеклова и методы разделения области в вариационных задачах // Вычислительные процессы и системы. Вып. 2. М.: Наука, 1985. С. 173-226.

17. Обэн Ж.-П. Приближенное решение эллиптических краевых задач. М.: Мир, 1977.

18. Михайлов В. П. Дифференциальные уравнения в частных производных. М.: Наука, 1983.

19. Ректорис K. Вариационные методы в математической физике и технике. М.: Мир, 1985.

20. Соболев С. Л. Некоторые применения функционального анализа в математической физике. Л.: Изд-во ЛГУ, 1950.

21. Gagliardo E. Caratterizzazione delle trace sulla frontiera relative ad alcune classi di funzioni in $n$ variabili // Rend. Sem. Mathem. univ. di Pavoda. 1957. V. 27. P. 284-305.

22. Слободецкий Л. Н. Обобщенные пространства С. Л. Соболева и их приложения к краевым задачам для дифференциальных уравнений в частных производных // Учен. зап. Ленингр. гос. пед. ин-та. 1958. Т. 197. С. 54-112.

23. Яковлев Г. Н. О следах функций из пространства $W_{p}^{l}$ на кусочно-гладких поверхностях // Матем. сб. 1967. Т. 74 (116). №4. С. 526-543.

24. Adams R. Sobolev spaces. New York-San Francisco-London: Academic Press, 1975.

25. Никольский C. M. Приближение функций многих переменных и теоремы вложения. М.: Наука, 1977.

26. Мазья В. Г. Пространства С. Л. Соболева. Л.: Изд-во ЛГУ, 1985.

27. Rademacher $H$. Über partielle und totale Differenzirbarkeit von Funktionen mehreren Variablen und über die Transformation der Doppelintegrale // Math. Ann. 1918. V. 79. P. 340-359.

28. Кружков C. Н. К вопросу о дифференцируемости почти всюду функций многих переменных // Вестн. МГУ. 1976. №6. С. 67-70.

29. Владимиров В. С. Обобщенные функции в математической физике. М.: Наука, 1981.

30. Рисс Ф., Секефальви-Надь Б. Лекции по функциональному анализу. М.: Мир, 1979.

31. Крейн С. Г. Линейные уравнения в банаховом пространстве. М.: Наука, 1971.

32. Березанский Ю. М. Разложения по собственньм функциям самосопряженных операторов. Киев: Наукова думка, 1965.

33. Agranovich M.S. Nonself-ajoint elliptic operators on nonsmooth domains // Russian J. Math. Phys. 1994. V. 2. №2. P. 139-148.

Вычислительньй центр РАН

Поступила в редакцию 10.01.1995 и 08.09 .1995 